\title{
EVANS FUNCTIONS, JOST FUNCTIONS, AND FREDHOLM DETERMINANTS
}

\author{
FRITZ GESZTESY, YURI LATUSHKIN, AND KONSTANTIN A. MAKAROV
}

\begin{abstract}
The principal results of this paper consist of an intrinsic definition of the Evans function in terms of newly introduced generalized matrix-valued Jost solutions for general first-order matrix-valued differential equations on the real line, and a proof of the fact that the Evans function, a finite-dimensional determinant by construction, coincides with a modified Fredholm determinant associated with a Birman-Schwinger-type integral operator up to a nonvanishing factor.
\end{abstract}

\section{INTRODUCTION}

In this paper we study connections between the asymptotic behavior of solutions of the following first-order $d \times d$ matrix-valued systems of linear differential equations on the real line $\mathbb{R}$,

$$
\begin{gathered}
y^{\prime}(x)=A(x) y(x), \quad x \in \mathbb{R}, \\
y^{\prime}(x)=(A(x)+R(x)) y(x), \quad x \in \mathbb{R},
\end{gathered}
$$

which will often be referred to as the unperturbed and perturbed equation in the following.

Three types of results are obtained:

- First, we establish the existence of special matrix-valued solutions of the perturbed equation (1.2), called the generalized matrix-valued Jost solutions, that are asymptotic to some reference solutions of the unperturbed equation (1.1). In spirit, these results resemble the celebrated Levinson Theorem [13, Theorem 1.3.1]; however, we are using a different approach involving Bohl and Lyapunov exponents.

- Second, we use the determinant of a finite-dimensional matrix composed of initial data of the generalized matrix-valued Jost solutions to calculate an infinitedimensional modified Fredholm determinant of a Birman-Schwinger-type integral operator associated with equations (1.1) and (1.2). These results generalize a classical relation identifying the Jost function and a Fredholm determinant in the case of one-dimensional half-line Schrödinger operators first derived by Jost and Pais [36].

- Third, using the generalized matrix-valued Jost solutions, we give a coordinatefree definition of the Evans function, and relate the Evans function to the abovementioned finite-dimensional determinant. Simultaneously, this identifies the Evans

Date: November 2, 2018.

2000 Mathematics Subject Classification. Primary: 47B10, 47G10, Secondary: 34B27, 34L40.

Key words and phrases. Fredholm determinants, non-self-adjoint operators, Jost functions, Evans function, asymptotic solutions, linear stability, travelling waves.

Based upon work supported by the US National Science Foundation under Grant Nos. DMS0405526, DMS-0338743, and DMS-0354339, and by the CRDF grant UP1-2567-OD-03. 
function with an infinite-dimensional modified Fredholm determinant associated with a Birman-Schwinger-type integral operator associated with (1.1) and (1.2) up to a nonvanishing factor. As a result, the Evans function is expressed via an infinite-dimensional modified Fredholm determinant, and, for the special case of the Schrödinger equation, is proved to be equal to the classical Jost function familiar in scattering theory.

The Evans function, $E$, is a Wronskian-type analytic function which is widely used to trace the spectrum of ordinary differential operators, $\mathcal{L}$. Most frequently, the operators $\mathcal{L}$ appear as linearizations of partial differential equations along special solutions such as travelling waves and steady states. For instance, $\mathcal{L}$ can be the one-dimensional Schrödinger operator, $d^{2} / d x^{2}+V$, obtained by linearizing the reaction-diffusion equation, $u_{t}=u_{x x}+r(u)$, about a steady state $u_{0}$, so that $V(x)=r^{\prime}\left(u_{0}(x)\right), x \in \mathbb{R}$.

Recently, the Evans function became one of the most important tools in stability analysis. It was originally introduced by J. W. Evans [15]-[18] to treat a particular model of nerve impulses, however, quite soon this object was generalized, and numerous connections of the Evans function to many fields of mathematical physics have been discovered (from Chern numbers in topology [1] to scattering data in quantum mechanics [39]). To give the reader just a small sample of the work done in this rapidly developing area, we cite, for instance, [1], [2], [4], [5], [11], [19], [20], [21], [22], [23], [24], [32], [34], [35], [37], [38], [39], [40], [42], [43], [47], [50], [51], [52], [54], [58], [59], [60], [61], [62], [67], [68], [69], and [70]. In addition, excellent reviews of this subject and further references can be found in [35] and [57].

Re-writing the eigenvalue problem for $\mathcal{L}$ as a first-order system of differential equations,

$$
y^{\prime}(x)=B(z, x) y(x), \quad x \in \mathbb{R},
$$

where $z \in \mathbb{C}$ is the spectral parameter, one is interested in conditions under which this system has $\mathbb{C}^{d}$-valued solutions $y$ on $\mathbb{R}$ exponentially decaying at $\pm \infty$. The Evans function, $E=E(z)$, is defined in such a way that $E(z)=0$ if and only if decaying $\mathbb{C}^{d}$-valued solutions $y$ exist (thus detecting the eigenvalues of $\mathcal{L}$ ). Since in this paper we are not concerned with function theoretic properties of the Evans function such as its analyticity, etc., deferring this topic to a forthcoming publication, we simply fix a value $z_{0}$ of $z$ and study equations (1.1) and (1.2) (and hence (1.3) with $\left.B\left(z_{0}, x\right)=A(x)+R(x), x \in \mathbb{R}\right)$. We note that, according to all definitions available in the literature, the Evans function for (1.1) and (1.2) is not uniquely defined, and one of the central issues in the current paper is to motivate a "canonical" choice of the definition of $E$.

To give an informal outline ${ }^{1}$ of our results, let us temporarily assume for simplicity of exposition, that $A$ and $R$ belong to $L^{\infty}(\mathbb{R})^{d \times d}$, and, in addition, that $R \in L^{1}(\mathbb{R})^{d \times d}$. On the Hilbert space $L^{2}(\mathbb{R})^{d}$ consider operators, $G_{A}$ and $G_{A+R}$, with domains $H^{1}(\mathbb{R})^{d}$, the usual Sobolev space, defined by

$$
\begin{aligned}
\left(G_{A} u\right)(x) & =-u^{\prime}(x)+A(x) u(x), \quad x \in \mathbb{R}, \\
\left(G_{A+R} u\right)(x) & =-u^{\prime}(x)+(A(x)+R(x)) u(x), \quad x \in \mathbb{R} .
\end{aligned}
$$

Next, we represent the perturbation $R$ as a product

$$
R(x)=R_{\ell}(x) R_{r}(x), \quad x \in \mathbb{R} .
$$

\footnotetext{
${ }^{1}$ See the glossary of notation at the end of this introduction.
} 
Assuming the invertibility of $G_{A}$ and $G_{A+R}$, one can write

$$
G_{A+R}^{-1}=G_{A}^{-1}-G_{A}^{-1} R_{\ell} R_{r} G_{A+R}^{-1}
$$

and, in addition, one can show that the operator $I+R_{r} G_{A}^{-1} R_{\ell}$ is invertible. Multiplying (1.6) from the left with $R_{r}$ and solving for $R_{r} G_{A+R}^{-1}$ then yields

$$
G_{A+R}^{-1}=G_{A}^{-1}-G_{A}^{-1} R_{\ell}\left[I+R_{r} G_{A}^{-1} R_{\ell}\right]^{-1} R_{r} G_{A}^{-1} .
$$

Conversely, assuming the invertibility of $G_{A}$ and $I+R_{r} G_{A}^{-1} R_{\ell}$, we conclude that $G_{A+R}$ is invertible and (1.7) holds. Next, still assuming that $G_{A}$ is invertible, one computes

$$
\begin{aligned}
G_{A}^{-1 / 2} G_{A+R} G_{A}^{-1 / 2} & =G_{A}^{-1 / 2}\left(G_{A}+R_{\ell} R_{r}\right) G_{A}^{-1 / 2} \\
& =I+G_{A}^{-1 / 2} R_{\ell} R_{r} G_{A}^{-1 / 2},
\end{aligned}
$$

and assuming that $R_{\ell} G_{A}^{-1 / 2}$ and $R_{r} G_{A}^{-1 / 2}$ are operators in $\mathcal{B}_{4}\left(L^{2}(\mathbb{R})^{d}\right.$ ) (for this it suffices that $R_{\ell}, R_{r} \in L^{2}(\mathbb{R})^{d \times d}$, cf. Lemma 2.9 ; also, see [30, 31, 66] for general information about the Schatten-von Neumann ideals $\left.\mathcal{B}_{p}(\cdot)\right)$, one obtains for the symmetrized perturbation determinant

$$
\begin{aligned}
\operatorname{det}_{2}\left(G_{A}^{-1 / 2} G_{A+R} G_{A}^{-1 / 2}\right) & =\operatorname{det}_{2}\left(I+G_{A}^{-1 / 2} R_{\ell} R_{r} G_{A}^{-1 / 2}\right) \\
& =\operatorname{det}_{2}\left(I+R_{r} G_{A}^{-1} R_{\ell}\right) .
\end{aligned}
$$

Here $\operatorname{det}_{2}(I+T)$ denotes the modified Fredholm determinant of a Hilbert-Schmidt operator $T$ on $L^{2}(\mathbb{R})^{d}$ (and we used the fact that $\operatorname{det}_{2}\left(I+T_{1} T_{2}\right)=\operatorname{det}_{2}\left(I+T_{2} T_{1}\right)$ for $T_{1}, T_{2}$ bounded operators such that $T_{1} T_{2}$ and $T_{2} T_{1}$ are Hilbert-Schmidt). In removing the restriction $A, R \in L^{\infty}(\mathbb{R})^{d \times d}$ and permitting $A$ to be locally integrable on $\mathbb{R}$ one needs to supply proper operator closures in formulas (1.6)-(1.9) (see, e.g., [27] and the extensive literature therein for a more general discussion of factorizations of perturbations).

To make the connection with (1.1) and (1.2) we recall that the operator $G_{A}$ is invertible on $L^{2}(\mathbb{R})^{d}$ if and only if (1.1) has an exponential dichotomy $Q$ on $\mathbb{R}$ (see, e.g., [7, Ch. 3]). By a classical result in the theory of dichotomic differential equations (see, e.g., [8, Proposition 4.1], [10, Ch. IV]), since (1.1) has an exponential dichotomy on $\mathbb{R}$ and the perturbation satisfies $\|R\|_{\mathbb{C}^{d \times d}} \in L^{1}(\mathbb{R})$, one infers that the perturbed equation (1.2) has exponential dichotomies on both half-lines $\mathbb{R}_{+}$ and $\mathbb{R}_{-}$, and moreover (by yet another well-known Dichotomy Theorem, see [3], [48], [49], [56]), the operator $G_{A+R}$ is then a Fredholm operator with zero Fredholm index. Thus, $G_{A+R}$ is either invertible on $L^{2}(\mathbb{R})^{d}$, or has a nontrivial null space (consisting exactly of the solutions of $(1.2)$ that belong to $\left.H^{1}(\mathbb{R})^{d}\right)$.

Due to the assumption $\|R\|_{\mathbb{C}^{d \times d}} \in L^{1}(\mathbb{R})$, the Birman-Schwinger-type operator

$$
K=R_{r} G_{A}^{-1} R_{\ell}
$$

is a Hilbert-Schmidt operator on $L^{2}(\mathbb{R})^{d}$ (see Lemma 2.9). Thus, employing the celebrated Birman-Schwinger argument we conclude from (1.7) that the operator $G_{A+R}$ is not invertible (that is, $(1.2)$ has solutions in $H^{1}(\mathbb{R})^{d}$ ) if and only if -1 is an eigenvalue of $K$, or, in other words, if and only if the 2-modified perturbation determinant $\operatorname{det}_{2}(I+K)$ is equal to zero.

Without going into further details at this point, we mention that the BirmanSchwinger-type argument (cf. [55, Sect. X.III.3] for this terminology) in our present context provides a bijection between the geometric eigenspace (nullspace) of $G_{A+R}$ 
and the geometric eigenspace of $R_{r} G_{A}^{-1} R_{\ell}$ corresponding to the eigenvalue -1 (see, e.g., [25], [27], [55, Sect. XIII.3], [63, Ch. III], [64] and the recent discussion in [27] which particularly targets non-self-adjoint operators).

Equation (1.9) finally illustrates the sense in which $\operatorname{det}_{2}(I+K)$ can be viewed as a properly symmetrized modified perturbation determinant (cf. [31, Sect. IV.3]) associated with the pair $\left(G_{A}, G_{A+R}\right)$. We note at this point that $\operatorname{det}_{2}(I+K)$ will be one of the central objects in this paper.

Our next step is to reduce the calculation of the infinite-dimensional determinant $\operatorname{det}_{2}(I+K)$ to a finite-dimensional determinant. This is possible because the integral kernel of the operator $K$ happens to be of a special type: it belongs to the class of so-called semi-separable integral kernels (cf. [28], [29], [30, Ch. XIII]). For the integral operator with semi-separable integral kernel we construct a finitedimensional determinant, $D$, such that the following representation holds:

$$
\operatorname{det}_{2}(I+K)=e^{\Theta} D,
$$

where $\Theta$ is a certain constant. It is known from the work in [28] that $D$ can be expressed in terms of solutions of certain Volterra integral equations associated with (1.1) and (1.2). We make yet another step, and express $D$ in terms of solutions of (1.2) that are asymptotic to some reference solutions of (1.1). We called these solutions of (1.2) the generalized matrix-valued Jost solutions, since they appear as a generalization of the classical Jost solutions of the Schrödinger equation $[6$, Ch. XVII], [46, Sect. 12.1]. In particular, we prove that the determinant $D$ in (1.11) is equal to the classical Jost function in the case of the perturbed equation (1.2) corresponding to the eigenvalue problem for the Schrödinger operator $\mathcal{L}$. The reference solutions are chosen using the Lyapunov exponents while the rate of approximation of the reference solutions by the generalized matrix-valued Jost solutions is controlled by the Bohl exponents for (1.1) (see the terminology in [10, Ch. III]). The existence of the generalized matrix-valued Jost solutions is proved by a contraction mapping argument applied to a "mixed" system of integral equations of Volterra- and Fredholm-type (which appears to be a new element in the literature). In addition, we identify the abstract asymptotic properties of the generalized matrix-valued Jost solutions leading to formula (1.11). Finally, we complete the picture by proving that the finite-dimensional determinant $D$ in (1.11) is equal to the Evans function when $E$ is defined by means of the initial data of the generalized matrix-valued Jost solutions, thus making this choice of $E$ canonical.

We emphasize that our results on the existence of the generalized matrix-valued Jost solutions can be viewed (cf. Remarks 7.11 and 8.5) as a further refinement of the celebrated Levinson theorem (see, for instance, [13, Theorem 1.3.1]).

Some results of the current paper have been announced in [26]. A version of this theory for difference equations, based on the material developed in this paper, has been derived in [9].

The paper is organized as follows. In Section 2 we recall some known facts from $[8,10]$ regarding exponential dichotomies and the Bohl and Lyapunov exponents (which are our means to measure the asymptotic behavior of solutions of differential equations), and also show that $K$ is a Hilbert-Schmidt operator on $L^{2}(\mathbb{R})^{d}$ (Lemma 2.9). In addition, we formulate Lemmas 2.13 and 2.14 (proved in Appendix B) to the effect that the exponential dichotomy on half-lines and, more generally, exponential splittings and the corresponding Bohl exponents for (1.1) persist under $L^{1}$-perturbations. Note that the robustness of the Bohl exponents can also be 
proved under assumptions different from $\|R\|_{\mathbb{C}^{d \times d}} \in L^{1}(\mathbb{R})$ (e.g., assuming that the perturbation is continuous and decays at $\pm \infty$ to zero in norm), and thus our subsequent results can be developed in this different setting, but to keep this manuscript at a reasonable length we do not pursue this in the current paper.

In Section 3 we introduce in Definition 3.1 the matrix-valued Jost solutions, $Y_{ \pm}$ on $\mathbb{R}_{ \pm}$, which are proved to exist (and to be unique) under the assumptions that (1.1) has an exponential dichotomy on $\mathbb{R}$ and $\|R\|_{\mathbb{C}^{d \times d}}$ decays exponentially at $\pm \infty$ at a rate controlled by the width of the two Bohl segments corresponding to the dichotomy projections (cf. Theorems 3.2 and 3.8). Although this result is obtained under strong exponential decay assumptions, it is shown to be optimal if one does not allow further exponential splitting of the dichotomy projections (cf. Example 3.6).

In Section 4 (cf. Theorem 4.1) we establish formula (1.11) with

$$
D=\operatorname{det}_{\mathbb{C}^{d}}\left(Y_{+}(0)+Y_{-}(0)\right)
$$

under the assumptions of Section 3. We note that these assumptions are satisfied for compactly supported perturbations, and our method of proof of (1.11) is to establish this formula for a truncated perturbation first, and then pass to the limit as the support of the truncation expands. Formula (1.11) in the compactly supported perturbation case (which is our main intermediate calculation) is proved using results from [28] collected in Appendix A. We call $D$ in (1.11) the Evans determinant.

Section 5 deals with the class of perturbed equations (1.2), where each of the Bohl segments for the dichotomy projections degenerates to a single point (cf. Theorem 5.3). In particular, this assumption covers the case of (1.2) corresponding to the Schrödinger equation, and in this section we also show that our matrix-valued Jost solutions indeed generalize the classical Jost solutions, and, moreover, that the Evans determinant $D$ in (1.11) coincides with the Jost function (see Theorem 5.4). We refer to $[39,40]$ for results relating the Evans function and a Fredholm determinant in the Schrödinger equation case.

In Sections 6 and 7 we introduce and study the generalized matrix-valued Jost solutions, assuming that the exponential dichotomy for (1.1) admits further (and finer) exponential splitting (of order two in Section 6 and of an arbitrary order in Section 7). The generalized matrix-valued Jost solutions are defined abstractly as solutions of (1.2) that approximate decaying reference solutions of (1.1) at $\pm \infty$ at an exponential rate which is better than the rate of decay of the reference solutions themselves (cf. Definitions 6.2 and 7.2). Unlike the matrix-valued Jost solutions from Section 3, the generalized matrix-valued Jost solutions are not unique due to the possible appearance of "lower-order modes", that is, solutions of the perturbed equation (1.2) having worse exponential rate of decay than the corresponding reference solutions. However, they are unique up to terms that decay exponentially faster than the respective reference solutions (see Corollary 6.6 and Theorem 7.3). Nevertheless, we prove in Lemma 7.6 that the Evans determinant $D$ defined by means of the generalized matrix-valued Jost solutions is determined uniquely. The existence of the generalized matrix-valued Jost solutions is proved using a "mixed" system of integral equations of Volterra- and Fredholm-type in Theorems 6.5 and 7.3. The use of the finer exponential splitting allows us to prove the existence of the generalized matrix-valued Jost solutions and formula (1.11) under much weaker assumptions on the exponential decay of the perturbation than those in Section 3: 
In Sections 6 and 7 the decay of the perturbation is controlled by the width of each of the Bohl segments corresponding to the fine exponential splitting (cf. Theorems 6.5 and 7.8). This constitutes one of the main new effects observed in this paper: Passing from the case of two- to the case of many Bohl segments in the unperturbed equation (1.1) requires replacing the system of two Volterra integral equations by a cascade-type mixed system of Volterra- and Fredholm-type integral equations so that one can take care of the appearance of the above mentioned "lower-order modes". As a generalization in yet another direction, we express in Corollary 7.10 the infinite-dimensional determinant $\operatorname{det}_{2}(I+K)$ in (1.11) as a ratio of two Evans determinants corresponding to the perturbed and the unperturbed equation (1.1) thus making the connection with the interpretation of $\operatorname{det}_{2}(I+K)$ as a (modified) perturbation determinant (cf. [31, Sect. IV.3]).

In Section 8 we treat the special but important case of the constant coefficient unperturbed equation (1.1), $A(x)=A, x \in \mathbb{R}$. We introduce the generalized matrix-valued Jost solutions as solutions of the "mixed" system of Volterra- and Fredholm-type integral equations in Definition 8.2. Using the Jordan normal form of $A$, the existence of the solutions and formula (1.11) are proved under the following mild assumptions in Theorem 8.3: Either we suppose $\|R\|_{\mathbb{C}^{d \times d}} \in L^{1}(\mathbb{R})$ if the spectrum of $A$ is semi-simple, or we assume a polynomial decay of $\|R\|_{\mathbb{C}^{d \times d}}$. These hypotheses are shown to be optimal.

Finally, in Section 9 we show that the Evans determinant $D$ equals the Evans function for (1.1) and (1.2) defined via the generalized matrix-valued Jost solutions.

A glossary of notation: For $d, d_{1}, d_{2} \in \mathbb{N}$, let $\mathbb{C}^{d_{1} \times d_{2}}$ be the set of $d_{1} \times d_{2}$ matrices with complex entries; by $\operatorname{tr}_{\mathbb{C}^{d}}(M)$ and $\operatorname{det}_{\mathbb{C}^{d}}(M)$ we denote the trace and determinant of a $d \times d$ matrix $M \in \mathbb{C}^{d \times d}$. $\|\cdot\|_{\mathbb{C}^{d}}$ denotes a vector norm in $\mathbb{C}^{d}$; $\|\cdot\|_{\mathbb{C}^{d \times d}}$ a matrix norm in $\mathbb{C}^{d \times d} ;\|\cdot\|_{\mathcal{X}}$ a norm in a Banach space $\mathcal{X}$. For $p \geq 1$ and $J \subseteq \mathbb{R}, L^{p}(J), L^{p}(J)^{d}$, and $L^{p}(J)^{d \times d}$ are the usual Lebesgue spaces on $J$ with values in $\mathbb{C}, \mathbb{C}^{d}$, and $\mathbb{C}^{d \times d}$, associated with Lebesgue measure $d x$ on $J$. Similarly, $L^{p}(J ; w(x) d x), L^{p}(J ; w(x) d x)^{d}$ and $L^{p}(J ; w(x) d x)^{d \times d}$ are the weighted spaces with a weight $w \geq 0$; the spaces of bounded continuous functions on $J$ are denoted by $C_{\mathrm{b}}(J), C_{\mathrm{b}}(J)^{d}$ and $C_{\mathrm{b}}(J)^{d \times d}$, respectively. The identity matrix in $\mathbb{C}^{d \times d}$ is denoted by $I_{d}$ and the identity operator on a Banach space $\mathcal{X}$ is denoted by $I$ (or by $I_{\mathcal{X}}$ if its dependence on $\mathcal{X}$ needs to be stressed). For a projection $Q$ we write $\operatorname{ran}(Q)$ and $\operatorname{ker}(Q)$ to denote the range and the null space (kernel) of $Q$. If $T$ is a linear operator on a Banach space $\mathcal{X}$, then $T^{-1} \in \mathcal{B}(\mathcal{X})$ denotes the (bounded) inverse operator of $T$, with $\mathcal{B}(\mathcal{X})$ being the Banach space of bounded linear operators on $\mathcal{X}$. Moreover, $\sigma(T)$ denotes the spectrum of $T$, and $\left.T\right|_{\mathcal{Y}}$ denotes the restriction of $T$ to a subspace $\mathcal{Y}$ of $\mathcal{X}$. If $\mathcal{X}_{1}$ and $\mathcal{X}_{2}$ are two subspaces of $\mathcal{X}$, then $\mathcal{X}_{1} \dot{+} \mathcal{X}_{2}$ denotes their direct (but not necessarily orthogonal) sum. $\mathcal{B}_{p}(\mathcal{H})$ denotes the Schatten-von Neumann ideals of compact operators on a Hilbert space $\mathcal{H}$ with singular values in $\ell^{p}, p \geq 1$. A generic constant is denoted by $c$ or $C$, and we also use the abbreviations $\mathbb{R}_{+}=[0, \infty), \mathbb{R}_{-}=(-\infty, 0]$.

\section{Preliminaries}

Throughout this paper we make the following assumptions on $A$ and $R$ in (1.1) and (1.2): 
Hypothesis 2.1. Suppose

$$
A, R \in L_{\mathrm{loc}}^{1}(\mathbb{R})^{d \times d} .
$$

Let $\Phi$ denote the fundamental matrix solution of the unperturbed equation (1.1) so that $\Phi^{\prime}(x)=A(x) \Phi(x), x \in \mathbb{R}$ and $\Phi(0)=I_{d}$. Throughout, we assume that the propagator $\Phi(x) \Phi\left(x^{\prime}\right)^{-1}$ of (1.1) is exponentially bounded on $\mathbb{R}$, that is, we suppose that

$$
\sup _{x, x^{\prime} \in \mathbb{R},\left|x-x^{\prime}\right| \leq 1}\left\|\Phi(x) \Phi\left(x^{\prime}\right)^{-1}\right\|_{\mathbb{C}^{d \times d}}<\infty,
$$

or equivalently, there exist constants $C \in[1, \infty)$ and $\alpha \in \mathbb{R}$, such that

$$
\left\|\Phi(x) \Phi\left(x^{\prime}\right)^{-1}\right\|_{\mathbb{C}^{d \times d}} \leq C e^{\alpha\left|x-x^{\prime}\right|}, \quad x, x^{\prime} \in \mathbb{R} .
$$

First, we recall basic definitions and some standard facts regarding Bohl and Lyapunov exponents and dichotomies (see, e.g., [8, Sec.1], [10, Ch. III]).

A projection $Q$ in $\mathbb{C}^{d}$ is said to be uniformly conjugated by $\Phi$ on $\mathbb{R}$ if

$$
\sup _{x \in \mathbb{R}}\left\|\Phi(x) Q \Phi(x)^{-1}\right\|_{\mathbb{C}^{d \times d}}<\infty .
$$

The geometrical meaning of this condition is that the angle between the range and the null space of the projection $\Phi(x) Q \Phi(x)^{-1}$ in $\mathbb{C}^{d}$ is uniformly separated from zero for all $x \in \mathbb{R}$ (see, e.g., [10, Corollary IV.1.1]).

Let $Q$ be a projection in $\mathbb{C}^{d}$ which is uniformly conjugated by $\Phi$ on $\mathbb{R}$. The upper Bohl exponent on $\mathbb{R}$ associated with the projection $Q$, denoted by $\varkappa(Q)$, is defined as the infimum of all $\varkappa \in \mathbb{R}$, such that for some constant $C(\varkappa) \in[1, \infty)$, the following inequality holds for all $x, x^{\prime} \in \mathbb{R}$ such that $x \geq x^{\prime}$,

$$
\left\|\Phi(x) Q \Phi\left(x^{\prime}\right)^{-1}\right\|_{\mathbb{C}^{d \times d}} \leq C(\varkappa) e^{\varkappa\left(x-x^{\prime}\right)} .
$$

The lower Bohl exponent on $\mathbb{R}$, denoted by $\varkappa^{\prime}(Q)$, is defined as the supremum of all $\varkappa \in \mathbb{R}$, such that (2.5) holds for all $x \leq x^{\prime}$, where $x, x^{\prime} \in \mathbb{R}$.

Similarly, given a projection $Q$ which is uniformly conjugated by $\Phi$ on $\mathbb{R}_{+}$(resp., on $\mathbb{R}_{-}$), the upper Bohl exponent, $\varkappa_{+}(Q)$, and the lower Bohl exponent, $\varkappa_{+}^{\prime}(Q)$, on $\mathbb{R}_{+}$associated with the projection $Q$ (resp., the Bohl exponents $\varkappa_{-}(Q)$ and $\varkappa_{-}^{\prime}(Q)$ on $\mathbb{R}_{-}$), are defined in the same way except that one takes $x, x^{\prime} \in \mathbb{R}_{+}$(respectively, $\left.x, x^{\prime} \in \mathbb{R}_{-}\right)$in (2.5). The upper and lower Bohl exponents on $\mathbb{R}_{+}$can also be expressed as follows (see [10, Theorem III.4.4])

$$
\begin{aligned}
& \varkappa_{+}(Q)=\limsup _{\left(x-x^{\prime}\right) \rightarrow \infty, x^{\prime} \rightarrow \infty} \frac{\log \left\|\Phi(x) Q \Phi\left(x^{\prime}\right)^{-1}\right\|_{\mathbb{C}^{d \times d}}}{x-x^{\prime}}, \\
& \varkappa_{+}^{\prime}(Q)=-\limsup _{\left(x-x^{\prime}\right) \rightarrow \infty, x^{\prime} \rightarrow \infty} \frac{\log \left\|\Phi\left(x^{\prime}\right) Q \Phi(x)^{-1}\right\|_{\mathbb{C}^{d \times d}}}{x-x^{\prime}} ;
\end{aligned}
$$

similar formulas hold for $\varkappa_{-}(Q)$ and $\varkappa_{-}^{\prime}(Q)$.

We introduce the upper, $\lambda_{+}(Q)$, and the lower, $\lambda_{+}^{\prime}(Q)$, Lyapunov exponents on $\mathbb{R}_{+}$associated with the projection $Q$ by the formulas

$$
\lambda_{+}(Q)=\limsup _{x \rightarrow \infty} \frac{\log \|\Phi(x) Q\|_{\mathbb{C}^{d \times d}}}{x}, \quad \lambda_{+}^{\prime}(Q)=-\limsup _{x \rightarrow \infty} \frac{\log \left\|Q \Phi(x)^{-1}\right\|_{\mathbb{C}^{d \times d}}}{x},
$$


and the Lyapunov exponents on $\mathbb{R}_{-}$by the formulas

$$
\lambda_{-}(Q)=\limsup _{x \rightarrow-\infty} \frac{\log \left\|Q \Phi(x)^{-1}\right\|_{\mathbb{C}^{d \times d}}}{|x|}, \quad \lambda_{-}^{\prime}(Q)=-\limsup _{x \rightarrow-\infty} \frac{\log \|\Phi(x) Q\|_{\mathbb{C}^{d \times d}}}{|x|} .
$$

We remark that the upper (lower) Bohl exponent measures the best (worst) exponential growth of the propagator $\Phi(x) \Phi\left(x^{\prime}\right)^{-1}$ and the upper (lower) Lyapunov exponent measures the best (worst) exponential growth of the fundamental solution $\Phi$ relative to the projection $Q$. Under the assumption that the propagator of (1.1) is exponentially bounded, the Bohl exponents are finite [10, Theorem III.4.2]. We note the inequalities

$$
\varkappa^{\prime}(Q) \leq \varkappa_{ \pm}^{\prime}(Q) \leq \lambda_{ \pm}^{\prime}(Q) \leq \lambda_{ \pm}(Q) \leq \varkappa_{ \pm}(Q) \leq \varkappa(Q)
$$

and stress that they are strict in general (in particular, the possible inequality of the Bohl and Lyapunov exponents is exhibited in the classical Perron example, see, e.g., [10, Sect. III.4.4]).

Remark 2.2. The set of Bohl and Lyapunov exponents for the rescaled equation,

$$
y^{\prime}(x)=\left(A(x)-\mu I_{d}\right) y(x), \quad x \in \mathbb{R}, \mu \in \mathbb{C},
$$

with the associated propagator $e^{-\mu\left(x-x^{\prime}\right)} \Phi(x) \Phi\left(x^{\prime}\right)^{-1}, x, x^{\prime} \in \mathbb{R}$, is obtained from the set of the exponents of equation (1.1) by shifting the latter by the amount $-\operatorname{Re}(\mu)$.

Example 2.3. If the unperturbed equation (1.1) is autonomous then the Bohl and Lyapunov exponents are the same and equal to the real parts of the eigenvalues of $A$. To give more details and fix notations, suppose that $A(x)=A, x \in \mathbb{R}$, for some $A \in \mathbb{C}^{d \times d}$, and let $\nu_{k} \in \mathbb{C}, k=1, \ldots, d$, be the eigenvalues of $A$. We split the set of the eigenvalues as $\sigma(A)=\cup_{j=1}^{d^{\prime}} \Sigma_{j}$, where $1 \leq d^{\prime} \leq d$ and for each $j$ all eigenvalues that belong to $\Sigma_{j}$ have the same real part denoted by $\varkappa_{j}, j=1, \ldots, d^{\prime}$. Let $Q_{j}$ denote the spectral projection for $A$ such that $\sigma\left(\left.A\right|_{\operatorname{ran}\left(Q_{j}\right)}\right)=\Sigma_{j}$. Then the Bohl and the Lyapunov exponents on $\mathbb{R}$ (and on $\mathbb{R}_{ \pm}$) associated with $Q_{j}$, are equal to $\varkappa_{j}$ (we note that we will always enumerate $\varkappa_{j}$ in increasing order of magnitude, $\varkappa_{j}<\varkappa_{j^{\prime}}$ for $\left.j<j^{\prime}, j, j^{\prime}=1, \ldots, d^{\prime}\right)$.

Returning to the general nonautonomous case, equation (1.1) is said to have a bounded dichotomy $Q$ on $\mathbb{R}$ if $Q$ is a projection in $\mathbb{C}^{d}$ (called the dichotomy projection) so that for some constant $M \in[1, \infty)$, the following inequalities hold for all $x, x^{\prime} \in \mathbb{R}$ :

$$
\begin{aligned}
\left\|\Phi(x) Q \Phi\left(x^{\prime}\right)^{-1}\right\|_{\mathbb{C}^{d \times d}} \leq M, & x \geq x^{\prime}, \\
\left\|\Phi(x)\left(I_{d}-Q\right) \Phi\left(x^{\prime}\right)^{-1}\right\|_{\mathbb{C}^{d \times d}} \leq M, & x \leq x^{\prime} .
\end{aligned}
$$

Equation (1.1) is said to have an exponential dichotomy $Q$ on $\mathbb{R}$ if $Q$ is a projection in $\mathbb{C}^{d}$ so that for some positive constants $\varkappa$ and $\varkappa^{\prime}$, and some constants $C(\varkappa), C\left(\varkappa^{\prime}\right) \in$ $[1, \infty)$, the following inequalities hold for all $x, x^{\prime} \in \mathbb{R}$ :

$$
\begin{aligned}
\left\|\Phi(x) Q \Phi\left(x^{\prime}\right)^{-1}\right\|_{\mathbb{C}^{d \times d}} & \leq C(\varkappa) e^{-\varkappa\left(x-x^{\prime}\right)}, \quad x \geq x^{\prime}, \\
\left\|\Phi(x)\left(I_{d}-Q\right) \Phi\left(x^{\prime}\right)^{-1}\right\|_{\mathbb{C}^{d \times d}} & \leq C\left(\varkappa^{\prime}\right) e^{\varkappa^{\prime}\left(x-x^{\prime}\right)}, \quad x \leq x^{\prime} .
\end{aligned}
$$

We note that $Q$ is an exponential dichotomy for (1.1) on $\mathbb{R}$ if and only if the following inequalities for the Bohl exponents hold:

$$
\varkappa(Q)<0<\varkappa^{\prime}\left(I_{d}-Q\right) \text {. }
$$


Dichotomies on $\mathbb{R}_{+}$, respectively on $\mathbb{R}_{-}$, are defined in the same way except in (2.12) or (2.13), (2.14) one takes only $x, x^{\prime} \in \mathbb{R}_{+}$, respectively, $x, x^{\prime} \in \mathbb{R}_{-}$.

Example 2.4. Assume in Example 2.3 that $\sigma(A) \cap i \mathbb{R}=\emptyset$ and $d^{\prime} \geq 2$. Consider $k_{0} \in\left\{1, \ldots, d^{\prime}-1\right\}$ such that $\varkappa_{k_{0}}<0<\varkappa_{k_{0}+1}$. Then $Q=\sum_{j=1}^{k_{0}} Q_{j}$ is the exponential dichotomy projection for the equation $y^{\prime}(x)=A y(x)$ on $\mathbb{R}, \mathbb{R}_{+}$, and $\mathbb{R}_{-}$.

Definition 2.5. ([10, Sect. IV.4].) Assume $d \geq 2$, and let $1<d^{\prime} \leq d$. A system $\left\{Q_{j}\right\}_{j=1}^{d^{\prime}}$ of disjoint projections in $\mathbb{C}^{d}$ is called an exponential splitting for (1.1) on $\mathbb{R}$ if the following conditions hold:

(i) $\sum_{j=1}^{d^{\prime}} Q_{j}=I_{d}$.

(ii) Each projection $Q_{j}$ is uniformly conjugated by $\Phi$ on $\mathbb{R}$.

(iii) The segments $\left[\varkappa^{\prime}\left(Q_{j}\right), \varkappa\left(Q_{j}\right)\right]$ are disjoint.

The segments $\left[\varkappa^{\prime}\left(Q_{j}\right), \varkappa\left(Q_{j}\right)\right]$ of the real axis are called the Bohl segments associated with $Q_{j}$; they are determined by the lower and upper Bohl exponents on $\mathbb{R}$ associated with the projections $Q_{j}$, and in what follows they will always be numbered so that $\varkappa\left(Q_{j}\right)<\varkappa^{\prime}\left(Q_{j+1}\right), j=1, \ldots, d^{\prime}-1$. If $d^{\prime} \geq 2$ and (1.1) has an exponential dichotomy $Q$ on $\mathbb{R}$, then for some $k_{0}, 1 \leq k_{0}<d^{\prime}-1$, we have the following splitting:

$$
Q=\sum_{j=1}^{k_{0}} Q_{j} \text { and } I_{d}-Q=\sum_{j=k_{0}+1}^{d^{\prime}} Q_{j},
$$

so that $\varkappa\left(Q_{k_{0}}\right)<0<\varkappa^{\prime}\left(Q_{k_{0}+1}\right)$.

Replacing $\mathbb{R}$ by $\mathbb{R}_{+}$or $\mathbb{R}_{-}$in Definition 2.5 , one can also consider exponential splittings on $\mathbb{R}_{+}$or $\mathbb{R}_{-}$. We note that the exponential dichotomy projection $Q$ on $\mathbb{R}$ is uniquely defined, while exponential dichotomy projections on $\mathbb{R}_{+}$or $\mathbb{R}_{-}$are not. Indeed, for example, in the case of $\mathbb{R}_{+}$, the subspace $N_{s}$ of initial data of bounded solutions of (1.1) on $\mathbb{R}_{+}$is determined uniquely; to obtain an exponential dichotomy projection $Q$ on $\mathbb{R}_{+}$one can choose an arbitrary direct complement $N_{u}$ of the subspace $N_{s}$ in $\mathbb{C}^{d}$ so that $\mathbb{C}^{d}=N_{s} \dot{+} N_{u}$, and define the exponential dichotomy projection $Q$ such that $\operatorname{ran}(Q)=N_{s}$ and $\operatorname{ker}(Q)=N_{u}$ (see [10, Remark IV.3.4]). We recall that if $Q$ is an exponential dichotomy for (1.1) on $\mathbb{R}$, then $Q$ is also an exponential dichotomy on both semi-axes $\mathbb{R}_{+}$and $\mathbb{R}_{-}$. Similarly, an exponential splitting $\left\{Q_{j}\right\}_{j=1}^{d^{\prime}}$ on $\mathbb{R}$ induces exponential splittings on $\mathbb{R}_{+}$and $\mathbb{R}_{-}$; we stress that in view of (2.10), the Bohl segments for $\mathbb{R}$ are, generally, wider than the segments for $\mathbb{R}_{+}$and $\mathbb{R}_{-}$. This may happen since an exponential dichotomy on $\mathbb{R}_{+}$does not necessarily imply an exponential dichotomy on $\mathbb{R}$.

Example 2.6. In Examples (2.3) and (2.4) each Bohl segment $\left[\varkappa^{\prime}\left(Q_{j}\right), \varkappa\left(Q_{j}\right)\right]$ associated with $Q_{j}$ degenerates into a single point $\varkappa_{j}, j=1, \ldots, d^{\prime}$, while the Bohl segments associated with the (exponential dichotomy) splitting $\left\{Q, I_{d}-Q\right\}$ are given by $\left[\varkappa_{1}, \varkappa_{k_{0}}\right]$ and $\left[\varkappa_{k_{0}+1}, \varkappa_{d^{\prime}}\right]$; we recall that in this case

$$
\begin{aligned}
& \varkappa_{1}=\inf \{\operatorname{Re}(\nu) \in \mathbb{R} \mid \nu \in \sigma(A)\}, \quad \varkappa_{k_{0}}=\sup \{\operatorname{Re}(\nu) \in \mathbb{R} \mid \nu \in \sigma(A), \operatorname{Re}(\nu)<0\}, \\
& \varkappa_{k_{0}+1}=\inf \{\operatorname{Re}(\nu) \in \mathbb{R} \mid \nu \in \sigma(A), \operatorname{Re}(\nu)>0\}, \quad \varkappa_{d^{\prime}}=\sup \{\operatorname{Re}(\nu) \in \mathbb{R} \mid \nu \in \sigma(A)\} .
\end{aligned}
$$


Remark 2.7. The Lyapunov exponents for nonzero $\mathbb{C}^{d}$-valued solutions $y=y(x)$ of (1.1) are defined as follows:

$$
\lambda_{ \pm}(y)=\limsup _{x \rightarrow \pm \infty} \frac{\log \left(\|y(x)\|_{\mathbb{C}^{d}}\right)}{x}, \quad \lambda_{ \pm}^{\prime}(y)=\liminf _{x \rightarrow \pm \infty} \frac{\log \left(\|y(x)\|_{\mathbb{C}^{d}}\right)}{x} .
$$

For any nonzero $\mathbb{C}^{d}$-valued solution $y$ of (1.1), and for any given exponential splitting $\left\{Q_{k}\right\}_{k=1}^{d^{\prime}}$, there exists a $j \in\left\{1, \ldots, d^{\prime}\right\}$ such that all four Lyapunov exponents $\lambda_{ \pm}(y), \lambda_{ \pm}^{\prime}(y)$ belong to the $j$ th Bohl segment. One verifies that the actual $j$ th Bohl segment depends on the initial data $y_{0}=y(0) \neq 0$ of the solution $y$ of (1.1), say, on $\mathbb{R}_{+}$, as follows:

$$
\begin{aligned}
& \text { If } y_{0} \in \operatorname{ran}\left(\sum_{k=1}^{j} Q_{k}\right) \text { then } \lambda_{+}(y) \leq \lambda_{+}\left(Q_{j}\right), \\
& \text { if } y_{0} \in \operatorname{ran}\left(\sum_{k=1}^{j} Q_{k}\right) \backslash \operatorname{ran}\left(\sum_{\ell=1}^{j-1} Q_{\ell}\right) \text { then } \lambda_{+}^{\prime}(y) \geq \lambda_{+}^{\prime}\left(Q_{j}\right), \\
& \text { if } \lambda_{+}(y)<\lambda_{+}^{\prime}\left(Q_{j}\right) \text { then } y_{0} \in \operatorname{ran}\left(\sum_{k=1}^{j-1} Q_{k}\right), \\
& \text { if } \lambda_{+}^{\prime}(y)>\lambda_{+}\left(Q_{j}\right) \text { then } y(0) \in \mathbb{C}^{d} \backslash \operatorname{ran}\left(\sum_{k=1}^{j} Q_{k}\right),
\end{aligned}
$$

where $j \in\left\{1, \ldots, d^{\prime}\right\}$ and we set $Q_{0}=0$. Similar assertions hold for the Laypunov exponents on $\mathbb{R}_{-}$.

For future references, we record the assumptions on the coefficient $A$ of the unperturbed equation (1.1).

Hypothesis 2.8. Assume that $A \in L_{\mathrm{loc}}^{1}(\mathbb{R})^{d \times d}$. In addition, we suppose that the propagator $\Phi(x) \Phi\left(x^{\prime}\right)^{-1}, x, x^{\prime} \in \mathbb{R}$, is exponentially bounded on $\mathbb{R}$, that is,

$$
\sup _{x, x^{\prime} \in \mathbb{R},\left|x-x^{\prime}\right| \leq 1}\left\|\Phi(x) \Phi\left(x^{\prime}\right)^{-1}\right\|_{\mathbb{C}^{d \times d}}<\infty,
$$

and that (1.1) has an exponential dichotomy $Q$ on $\mathbb{R}$.

Turning to the perturbed equation (1.2), we assume that the perturbation $R$ in (1.2) satisfies the condition

$$
\|R\|_{\mathbb{C}^{d \times d}} \in L^{1}(\mathbb{R}) .
$$

Let $U$ and $|R|$ denote the $d \times d$ matrices in the polar decomposition of $R$ :

$$
R(x)=U(x)|R(x)|, \quad|R(x)|=\left(R(x)^{*} R(x)\right)^{1 / 2}, \quad x \in \mathbb{R} .
$$

Throughout this paper we will use the notation

$$
R_{\ell}(x)=U(x)|R(x)|^{\frac{1}{2}}, \quad R_{r}(x)=|R(x)|^{\frac{1}{2}}, \quad x \in \mathbb{R},
$$

so that

$$
R(x)=R_{\ell}(x) R_{r}(x), \quad x \in \mathbb{R} .
$$


Lemma 2.9. Assume Hypothesis 2.8 and condition (2.21). Then the integral operator $K$ with integral kernel given by

$$
K\left(x, x^{\prime}\right)=\left\{\begin{array}{ll}
-R_{r}(x) \Phi(x) Q \Phi\left(x^{\prime}\right)^{-1} R_{\ell}\left(x^{\prime}\right), & x \geq x^{\prime}, \\
R_{r}(x) \Phi(x)\left(I_{d}-Q\right) \Phi\left(x^{\prime}\right)^{-1} R_{\ell}\left(x^{\prime}\right), & x<x^{\prime},
\end{array} \quad x, x^{\prime} \in \mathbb{R},\right.
$$

is a Hilbert-Schmidt operator on $L^{2}(\mathbb{R})^{d}$. Moreover, the map

$$
\left\{\begin{aligned}
L^{1}(\mathbb{R})^{d \times d} & \rightarrow \mathcal{B}_{2}\left(L^{2}(\mathbb{R})^{d}\right) \\
R & \mapsto K
\end{aligned}\right.
$$

is continuous.

Proof. Since by hypothesis equation (1.1) has an exponential dichotomy $Q$ on $\mathbb{R}$, the projection $Q$ is also a bounded dichotomy on $\mathbb{R}$, and thus (2.12) holds. Using (2.12), the Hilbert-Schmidt norm of the integral operator $K$ can be estimated as follows:

$$
\begin{aligned}
\|K\|_{\mathcal{B}_{2}\left(L^{2}(\mathbb{R})^{d}\right)}= & \iint_{\mathbb{R} \times \mathbb{R}} d x d x^{\prime}\left\|K\left(x, x^{\prime}\right)\right\|_{\mathbb{C}^{d \times d}}^{2} \\
= & \int_{\mathbb{R}} d x \int_{-\infty}^{x} d x^{\prime}\left\|R_{r}(x) \Phi(x) Q \Phi\left(x^{\prime}\right)^{-1} R_{\ell}\left(x^{\prime}\right)\right\|_{\mathbb{C}^{d \times d}}^{2} \\
& +\int_{\mathbb{R}} d x \int_{x}^{\infty} d x^{\prime}\left\|R_{r}(x) \Phi(x)\left(I_{d}-Q\right) \Phi\left(x^{\prime}\right)^{-1} R_{\ell}\left(x^{\prime}\right)\right\|_{\mathbb{C}^{d \times d}}^{2} \\
\leq & M^{2} \int_{\mathbb{R}} d x \int_{\mathbb{R}} d x^{\prime}\|R(x)\|_{\mathbb{C}^{d \times d}}\left\|R\left(x^{\prime}\right)\right\|_{\mathbb{C}^{d \times d}} \\
= & M^{2}\left(\int_{\mathbb{R}} d x\|R(x)\|_{\mathbb{C}^{d \times d}}\right)^{2} .
\end{aligned}
$$

In the last estimate we used the inequalities

$$
\left\|R_{r}(x)\right\|_{\mathbb{C}^{d \times d}} \leq\|R(x)\|_{\mathbb{C}^{d \times d}}^{\frac{1}{2}}, \quad\left\|R_{\ell}(x)\right\|_{\mathbb{C}^{d \times d}} \leq\|R(x)\|_{\mathbb{C}^{d \times d}}^{\frac{1}{2}}, \quad x \in \mathbb{R},
$$

which follow from the spectral theorem for self-adjoint matrices. The continuity of the mapping in (2.26) holds because the mappings $R \mapsto R_{r}$ and $R \mapsto R_{\ell}$ are continuous from the Banach space $L^{1}(\mathbb{R})^{d \times d}$ to the Hilbert space $L^{2}(\mathbb{R})^{d \times d}$.

Remark 2.10. Since the propagator of (1.1) is exponentially bounded, the formula

$$
\left(E_{\Phi}^{t} u\right)(x)=\Phi(x-t) u(x-t), \quad u \in L^{2}(\mathbb{R})^{d}, \quad x \in \mathbb{R}, t \geq 0,
$$

defines a strongly continuous semigroup, $\left\{E_{\Phi}^{t}\right\}_{t \geq 0}$, on $L^{2}(\mathbb{R})^{d}$, called the evolution semigroup (see [7, Sect. 3.2] for a detailed discussion and extensive bibliography). It is well-known (see, e.g., [7, Theorem 3.17]) that the generator, $G$, of the semigroup in (2.29) has a bounded inverse if and only if (1.1) has an exponential dichotomy $Q$ on $\mathbb{R}$ and, if this is the case, the inverse operator $G^{-1}$ is an integral operator on $L^{2}(\mathbb{R})^{d}$ with integral kernel given by

$$
F\left(x, x^{\prime}\right)=\left\{\begin{array}{ll}
-\Phi(x) Q \Phi\left(x^{\prime}\right)^{-1}, & x \geq x^{\prime}, \\
\Phi(x)\left(I_{d}-Q\right) \Phi\left(x^{\prime}\right)^{-1}, & x<x^{\prime},
\end{array} \quad x, x^{\prime} \in \mathbb{R},\right.
$$

where $Q$ is the dichotomy projection. Thus, under the hypotheses of Lemma 2.9, the integral operator $K$ admits the representation $K=\overline{\mathcal{M}_{R_{r}} G^{-1} \mathcal{M}_{R_{\ell}}}$, where $\mathcal{M}_{R_{r}}$ 
and $\mathcal{M}_{R_{\ell}}$ denote the maximally defined operators of multiplication by the matrixvalued functions $R_{r}, R_{\ell} \in L^{2}(\mathbb{R})^{d \times d}$, and the operator $S=\mathcal{M}_{R_{r}} G^{-1} \mathcal{M}_{R_{\ell}}$ (with the bounded closure $\bar{S})$ is defined on the set of functions $u \in \operatorname{dom}\left(\mathcal{M}_{R_{\ell}}\right)$ such that $S u \in L^{2}(\mathbb{R})^{d \times d}$. We refer to [27] for a detailed discussion of Birman-Schwinger-type operators and the Birman-Schwinger principle.

Remark 2.11. Using the semigroup in (2.29) one can describe the exponential dichotomy projection $Q$ for (1.1) on $\mathbb{R}$ by means of the Riesz spectral projection for the operator $E_{\Phi}^{1}$, with $t=1$ in $(2.29)$, as follows (see [7, Theorems 3.13, 3.17]): Assume that the generator $G$ of the semigroup in $(2.29)$ is invertible on $L^{2}(\mathbb{R})^{d}$. By [7, Theorem 3.13], it follows that $E_{\Phi}^{1}$ has no spectrum on the unit circle. Let $\mathcal{Q}$ denote the spectral projection for $E_{\Phi}^{1}$ on $L^{2}(\mathbb{R})^{d}$ such that $\sigma\left(\left.E_{\Phi}^{1}\right|_{\operatorname{ran}(\mathcal{Q})}\right)$ is the part of the spectrum of $E_{\Phi}^{1}$ contained inside the unit disc. Theorem 3.17 in [7] states that $\mathcal{Q}$ is an operator of multiplication in $L^{2}(\mathbb{R})^{d}$ by a projection-valued function $Q(\cdot) \in C_{\mathrm{b}}(\mathbb{R})^{d \times d}$ and, in addition, $Q=Q(0)$ is the exponential dichotomy projection for $(1.1)$ on $\mathbb{R}$.

Remark 2.12. The proof of Lemma 2.9 shows that the assumption of an exponential dichotomy on $\mathbb{R}$ for (1.1) can be relaxed to require only a bounded dichotomy. Under this weaker assumption the generator $G$ of the semigroup in (2.29) may not be invertible (cf. Remark 2.10), or may not even be a Fredholm operator on $L^{2}(\mathbb{R})^{d}$. Indeed, by a well-known Dichotomy Theorem (sometimes called Palmer's theorem), the operator $G$ is Fredholm on $L^{2}(\mathbb{R})^{d}$ if and only if (1.1) has exponential dichotomies $Q_{-}$on $\mathbb{R}_{-}$and $Q_{+}$on $\mathbb{R}_{+}$; see [3], [48], [49], [56] or [57, Theorem 3.2], and also [41], [53] for more recent versions of the dichotomy theorem.

Next, we will discuss the Bohl exponents and exponential splittings for the perturbed equation (1.2). Since the propagator of (1.1) is exponentially bounded, and condition (2.21) holds, it follows from the variation of constants formula and Gronwall's inequality that the propagator of the perturbed equation (1.2) is also exponentially bounded (see, e.g., [33, Lemma IV.4.1]). Thus, the Bohl exponents for the perturbed equation (1.2) are finite.

We conclude this section with two technical results to the effect that first, the exponential dichotomy and exponential splitting of (1.1) on $\mathbb{R}_{+}$persist under $L^{1}\left(\mathbb{R}_{+}\right)^{d \times d}$-perturbations, and second, that the corresponding Bohl exponents do not change under these perturbations (similar facts hold for $\mathbb{R}_{-}$). A proof of the first part of these results can be found in [8, Proposition 4.1] or [10, Theorem IV.5.1]. However, we were not able to find the second part in the literature, and so we will briefly sketch a proof in Appendix B by modifying some of the arguments in [10, Ch. IV]. Lemma 2.13 is used in Section 6, while its direct generalization, Lemma 2.14, is used in Section 7.

Lemma 2.13. Assume that the propagator of the unperturbed equation (1.1) on $\mathbb{R}_{+}$is exponentially bounded, that the unperturbed equation (1.1) has an exponential dichotomy $Q$ on $\mathbb{R}_{+}$, and the perturbation $R$ satisfies $\|R\|_{\mathbb{C}^{d \times d}} \in L^{1}\left(\mathbb{R}_{+}\right)$. Then the perturbed equation (1.2) has an exponential dichotomy $P$ on $\mathbb{R}_{+}$such that

$$
\varkappa_{+}(P)=\varkappa_{+}(Q) \text { and } \varkappa_{+}^{\prime}\left(I_{d}-P\right)=\varkappa_{+}^{\prime}\left(I_{d}-Q\right),
$$

where $\varkappa_{+}(P)$ and $\varkappa_{+}^{\prime}\left(I_{d}-P\right)$ are the Bohl exponents for the perturbed equation $(1.2)$ on $\mathbb{R}_{+}$. Here, $P$ is the projection in $\mathbb{C}^{d}$ parallel to $\operatorname{ker}(Q)$ onto the subspace 
$N$ consisting of the values $y(0)$ at zero of all bounded solutions $y$ of $(1.2)$ on $\mathbb{R}_{+}$. In addition,

$$
\operatorname{dim}(\operatorname{ran}(P))=\operatorname{dim}(\operatorname{ran}(Q)) \text { and } \operatorname{dim}(\operatorname{ker}(P))=\operatorname{dim}(\operatorname{ker}(Q)) .
$$

Lemma 2.14. Assume that the propagator of the unperturbed equation (1.1) on $\mathbb{R}_{+}$ is exponentially bounded, the unperturbed equation (1.1) has an exponential splitting $\left\{Q_{j}\right\}_{j=1}^{d^{\prime}}, 1<d^{\prime} \leq d$, on $\mathbb{R}_{+}$, and the perturbation $R$ satisfies $\|R\|_{\mathbb{C}^{d \times d}} \in L^{1}\left(\mathbb{R}_{+}\right)$. Then, the perturbed equation (1.2) has an exponential splitting $\left\{P_{j}\right\}_{j=1}^{d^{\prime}}$ on $\mathbb{R}_{+}$such that

and

$$
\operatorname{dim}\left(\operatorname{ran}\left(P_{j}\right)\right)=\operatorname{dim}\left(\operatorname{ran}\left(Q_{j}\right)\right), \quad j=1, \ldots, d^{\prime},
$$

$$
\varkappa_{+}\left(P_{j}\right)=\varkappa_{+}\left(Q_{j}\right), \quad j=1, \ldots, d^{\prime},
$$

where $\varkappa_{+}\left(P_{j}\right)$ are the Bohl exponents for the perturbed equation (1.2) on $\mathbb{R}_{+}$.

\section{Matrix-Valued Jost Solutions}

In this section we discuss optimal conditions on the perturbation $R$ under which, assuming an exponential dichotomy of (1.1), one can establish existence and uniqueness of bounded matrix-valued solutions of (1.2) (we will call them matrix-valued Jost solutions) that are asymptotically close with respect to an exponential weight factor to bounded solutions of the unperturbed equation (1.1). These results are motivated by the study of the one-dimensional Schrödinger equation on the real axis,

$$
-u^{\prime \prime}(x)+V(x) u(x)=k^{2} u(x), \quad x \in \mathbb{R}, k \in \mathbb{C},
$$

with an integrable potential $V \in L^{1}(\mathbb{R} ; d x)$, where the Jost solutions, $u_{ \pm}(k, \cdot)$, are introduced as the solutions of (3.1) that are asymptotically close to the free plane waves: $u_{ \pm}(k, x) \underset{x \rightarrow \pm \infty}{\sim} e^{ \pm i k x}$ (see, e.g., [6, Ch. XVII]).

Definition 3.1. Under Hypothesis 2.8, $d \times d$ matrix-valued solutions $Y_{ \pm}$of the differential equation

$$
Y_{ \pm}(x)=(A(x)+R(x)) Y_{ \pm}(x), \quad x \in \mathbb{R}_{ \pm},
$$

with a locally integrable $d \times d$ matrix-valued function $R$, are called matrix-valued Jost solutions if

$$
\limsup _{x \rightarrow \infty} \frac{\log \left\|Y_{+}(x)-\Phi(x) Q\right\|_{\mathbb{C}^{d \times d}}}{x}<\varkappa_{+}^{\prime}(Q)
$$

and

$$
\liminf _{x \rightarrow-\infty} \frac{\log \left\|Y_{-}(x)-\Phi(x)\left(I_{d}-Q\right)\right\|_{\mathbb{C}^{d \times d}}}{x}>\varkappa_{-}\left(I_{d}-Q\right) .
$$

To motivate this definition, we first note that the Lyapunov exponents $\lambda_{+}(Q)$ and $\lambda^{\prime}+(Q)$ of the bounded matrix-valued solution $\Phi Q$ of (1.1) on $\mathbb{R}_{+}$belong to the Bohl segment $\left[\varkappa_{+}^{\prime}(Q), \varkappa_{+}(Q)\right]$. If $Y_{+}$is a matrix-valued solution of (1.2) satisfying (3.3), then $Y_{+}$is bounded and therefore Lemma 2.13 implies that its Lyapunov exponents belong to the same segment. Thus, the significance of (3.3) is that the solution $Y_{+}$approximates the solution $\Phi Q$ exponentially better than either of these solutions decays as $x \rightarrow \infty$.

Our first result shows the existence and uniqueness of the matrix-valued Jost solution $Y_{+}$on $\mathbb{R}_{+}$using a rather strong assumption on the exponential fall-off of the perturbation $R$. 
Theorem 3.2. Assume Hypothesis 2.8 and the condition

$$
\|R\|_{\mathbb{C}^{d \times d}} \in L^{1}\left(\mathbb{R}_{+} ; e^{\beta x} d x\right)
$$

for some constant $\beta>0$ satisfying the inequality

$$
\beta>\lambda_{+}(Q)-\varkappa_{+}^{\prime}(Q) .
$$

Then the perturbed differential equation (1.2) has a unique matrix-valued Jost solution $Y_{+}(x), x \geq 0$. Moreover, the map

$$
\left\{\begin{aligned}
L^{1}\left(\mathbb{R}_{+} ; e^{\beta x} d x\right)^{d \times d} & \rightarrow \mathbb{C}^{d \times d} \\
R & \mapsto Y_{+}(0)
\end{aligned}\right.
$$

is continuous.

Proof. We split the proof of Theorem 3.2 into three steps.

Step 1. Uniqueness. Suppose that $Y_{+, 1}$ and $Y_{+, 2}$ are two different $d \times d$ matrixvalued Jost solutions of (1.2), and introduce the nonzero solution $Y(x)=Y_{+, 1}(x)-$ $Y_{+, 2}(x), x \geq 0$. Using (3.3), we infer:

$$
\limsup _{x \rightarrow \infty} \frac{\log \|Y(x)\|_{\mathbb{C}^{d \times d}}}{x} \leq \max _{k=1,2}\left(\limsup _{x \rightarrow \infty} \frac{\log \left\|Y_{+, k}(x)-\Phi(x) Q\right\|_{\mathbb{C}^{d \times d}}}{x}\right)<\varkappa_{+}^{\prime}(Q) .
$$

This shows, in particular, that $Y$ is a bounded solution of (1.2). Since $\|R\|_{\mathbb{C}^{d \times d}} \in$ $L^{1}\left(\mathbb{R}_{+}\right)$by hypothesis, Lemma 2.13 yields an exponential dichotomy $P$ for $(1.2)$ such that $\varkappa_{+}^{\prime}(P)=\varkappa_{+}^{\prime}(Q)$. Since $Y$ is a bounded solution, $Y(0) \in \operatorname{ran}(P)$ and hence we arrive at the contradiction

$$
\limsup _{x \rightarrow \infty} \frac{\log \|Y(x)\|_{\mathbb{C}^{d \times d}}}{x} \geq \varkappa_{+}^{\prime}(P)=\varkappa_{+}^{\prime}(Q),
$$

proving the uniqueness part of the theorem.

Step 2. Existence. By hypothesis, (1.1) has an exponential dichotomy $Q$ on $\mathbb{R}_{+}$. Therefore, for any $\varepsilon>0$ there exists a constant $C(\varepsilon) \geq 1$ such that applying (2.5) in the definitions of $\varkappa_{+}^{\prime}(Q)$ and $\varkappa_{+}^{\prime}\left(I_{d}-Q\right)$, we obtain the estimate

$$
\begin{aligned}
& \left\|\Phi(x) \Phi\left(x^{\prime}\right)^{-1}\right\|_{\mathbb{C}^{d \times d}} \leq\left\|\Phi(x) Q \Phi\left(x^{\prime}\right)^{-1}\right\|_{\mathbb{C}^{d \times d}}+\left\|\Phi(x)\left(I_{d}-Q\right) \Phi\left(x^{\prime}\right)^{-1}\right\|_{\mathbb{C}^{d \times d}} \\
& \leq\left(e^{\left(\varkappa_{+}^{\prime}(Q)\right)\left(x-x^{\prime}\right)}+e^{\left(\varkappa_{+}^{\prime}\left(I_{d}-Q\right)\right)\left(x-x^{\prime}\right)}\right) f_{\varepsilon}\left(\left|x-x^{\prime}\right|\right), \quad 0 \leq x \leq x^{\prime},
\end{aligned}
$$

where we denoted

$$
f_{\varepsilon}(x)=C(\varepsilon) e^{\varepsilon x}, \quad x \geq 0 .
$$

Since $\varkappa_{+}^{\prime}(Q)<\varkappa_{+}^{\prime}\left(I_{d}-Q\right)$, we get the estimate

$$
\left\|\Phi(x) \Phi\left(x^{\prime}\right)^{-1}\right\|_{\mathbb{C}^{d \times d}} \leq 2 e^{\varkappa_{+}^{\prime}(Q)\left(x-x^{\prime}\right)} f_{\varepsilon}\left(\left|x-x^{\prime}\right|\right), \quad 0 \leq x \leq x^{\prime} .
$$

Next, fix $\varepsilon$ such that $0<2 \varepsilon<\beta-\left(\lambda_{+}(Q)-\varkappa_{+}^{\prime}(Q)\right)$, introduce the function

$$
p(x)=e^{\left(\lambda_{+}(Q)-\varkappa_{+}^{\prime}(Q)\right) x} f_{\varepsilon}^{2}(x)\|R(x)\|_{\mathbb{C}^{d \times d}}, \quad x \geq 0,
$$

and observe that $0 \leq p \in L^{1}\left(\mathbb{R}_{+}\right)$due to (3.5) and (3.6).

On the space $C_{\mathrm{b}}\left(\mathbb{R}_{+}\right)^{d \times d}$ we now define a Volterra integral operator, $V$, by

$$
(V Z)(x)=\int_{x}^{\infty} d x^{\prime} e^{-\lambda_{+}(Q)\left(x-x^{\prime}\right)} f_{\varepsilon}(x)^{-1} f_{\varepsilon}\left(x^{\prime}\right) \Phi(x) \Phi\left(x^{\prime}\right)^{-1} R\left(x^{\prime}\right) Z\left(x^{\prime}\right),
$$

and consider the corresponding Volterra integral equation

$$
Z(x)=Z^{(0)}(x)-(V Z)(x), \quad x \geq 0,
$$


where $Z^{(0)}$ is defined by

$$
Z^{(0)}(x)=e^{-\lambda_{+}(Q)(x)} f_{\varepsilon}(x)^{-1} \Phi(x) Q, \quad x \geq 0 .
$$

Using (3.12) and the inequality $\varkappa_{+}^{\prime}(Q)-\lambda_{+}(Q) \leq 0$ (cf. (2.10)), one derives the following estimate for the integral kernel of the integral operator $V$ :

$$
\begin{aligned}
& \left\|V\left(x, x^{\prime}\right)\right\|_{\mathbb{C}^{d \times d}} \leq 2 e^{\left(\varkappa_{+}^{\prime}(Q)-\lambda_{+}(Q)\right)\left(x-x^{\prime}\right)} f_{\varepsilon}(x)^{-1} f_{\varepsilon}\left(x^{\prime}\right) f_{\varepsilon}\left(\left|x-x^{\prime}\right|\right)\left\|R\left(x^{\prime}\right)\right\|_{\mathbb{C}^{d \times d}} \\
& \quad \leq 2 e^{\left(\varkappa_{+}^{\prime}(Q)-\lambda_{+}(Q)\right) x} f_{\varepsilon}(x)^{-1} e^{-\left(\varkappa_{+}^{\prime}(Q)-\lambda_{+}(Q)\right) x^{\prime}} f_{\varepsilon}^{2}\left(x^{\prime}\right)\left\|R\left(x^{\prime}\right)\right\|_{\mathbb{C}^{d \times d}} \\
& \quad=2 e^{\left(\varkappa_{+}^{\prime}(Q)-\lambda_{+}(Q)\right) x} f_{\varepsilon}(x)^{-1} p\left(x^{\prime}\right) \\
& \quad \leq 2 f_{\varepsilon}(x)^{-1} p\left(x^{\prime}\right), \quad 0 \leq x \leq x^{\prime} .
\end{aligned}
$$

Since $f_{\varepsilon}(x) \geq 1, x \in \mathbb{R}_{+}$, and $p \in L^{1}\left(\mathbb{R}_{+}\right)$, the estimate (3.18) shows that $V$ is a compact operator on $C_{\mathrm{b}}\left(\mathbb{R}_{+}\right)^{d \times d}$ with spectral radius equal to zero. Since the exponential decay of $\|\Phi Q\|_{\mathbb{C}^{d \times d}}$ is controlled by $\lambda_{+}(Q)$, we see that $Z^{(0)} \in C_{\mathrm{b}}\left(\mathbb{R}_{+}\right)^{d \times d}$. Thus, the integral equation (3.15) has a unique solution $Z \in C_{\mathrm{b}}\left(\mathbb{R}_{+}\right)^{d \times d}$ that can be obtained by the iteration process

$$
Z(x)=\sum_{j=1}^{\infty} Z^{(j)}(x), \quad Z^{(j)}(x)=\left(V Z^{(j-1)}\right)(x), \quad j \in \mathbb{N}, x \geq 0 .
$$

Moreover, from (3.15) and (3.17) it also follows that

$$
\begin{aligned}
& f_{\varepsilon}(x)\left\|Z(x)-Z^{(0)}(x)\right\|_{\mathbb{C}^{d \times d}} \\
& \quad \leq 2\|Z\|_{C_{\mathrm{b}}\left(\mathbb{R}_{+}\right)^{d \times d}} e^{\left(\varkappa_{+}^{\prime}(Q)-\lambda_{+}(Q)\right) x} \int_{x}^{\infty} d x^{\prime} p\left(x^{\prime}\right) .
\end{aligned}
$$

A straightforward computation shows that the function

$$
Y_{+}(x)=e^{\lambda_{+}(Q) x} f_{\varepsilon}(x) Z(x), \quad x \geq 0,
$$

yields a solution of the perturbed differential equation (1.2), and hence (3.20) and $p \in L^{1}\left(\mathbb{R}_{+}\right)$imply

$$
e^{-\varkappa_{+}^{\prime}(Q) x}\left\|Y_{+}(x)-\Phi(x) Q\right\|_{\mathbb{C}^{d \times d}} \underset{x \rightarrow \infty}{=} o(1) .
$$

In addition, using (3.5) and (3.6) again, we may choose $\delta>0$ sufficiently small such that the function $p_{\delta}(x)=e^{\delta x} p(x)$ is integrable on $\mathbb{R}_{+}$. If $x \leq x^{\prime}$, then $p\left(x^{\prime}\right) \leq e^{-\delta x} p_{\delta}\left(x^{\prime}\right)$, and thus (3.20) implies

$$
\begin{aligned}
& f_{\varepsilon}(x)\left\|Z(x)-Z^{(0)}(x)\right\|_{\mathbb{C}^{d \times d}} \\
& \quad \leq 2\|Z\|_{C_{\mathrm{b}}\left(\mathbb{R}_{+}\right)^{d \times d}} e^{\left(\varkappa_{+}^{\prime}(Q)-\lambda_{+}(Q)\right) x} e^{-\delta x} \int_{x}^{\infty} d x^{\prime} p_{\delta}\left(x^{\prime}\right) .
\end{aligned}
$$

This gives a better asymptotic relation than (3.22),

$$
e^{-\varkappa_{+}^{\prime}(Q) x}\left\|Y_{+}(x)-\Phi(x) Q\right\|_{\mathbb{C}^{d \times d}} \underset{x \rightarrow \infty}{=} o\left(e^{-\delta x}\right),
$$

leading to

$$
\limsup _{x \rightarrow \infty} \frac{\log \left\|Y_{+}(x)-\Phi(x) Q\right\|_{\mathbb{C}^{d \times d}}}{x} \leq \varkappa_{+}^{\prime}(Q)-\delta<\varkappa_{+}^{\prime}(Q),
$$

proving the existence part of the theorem. 
Step 3. Continuity. Let $\left\{R_{n}\right\}_{n=1}^{\infty}$ be a sequence of $d \times d$ matrix-valued functions converging to $R$ in $L^{1}\left(\mathbb{R}_{+} ; e^{\beta x} d x\right)^{d \times d}$ as $n \rightarrow \infty$. On the Banach space $C_{\mathrm{b}}\left(\mathbb{R}_{+}\right)^{d \times d}$ introduce Volterra integral operators $V_{n}$ by

$$
\left(V_{n} Z\right)(x)=\int_{x}^{\infty} d x^{\prime} e^{-\lambda_{+}(Q)\left(x-x^{\prime}\right)} f_{\varepsilon}(x)^{-1} f_{\varepsilon}\left(x^{\prime}\right) \Phi(x) \Phi\left(x^{\prime}\right)^{-1} R_{n}\left(x^{\prime}\right) Z\left(x^{\prime}\right), \quad n \in \mathbb{N} .
$$

An estimate similar to (3.18) shows that the sequence of the operators $V_{n}$ converges in operator norm to the integral operator $V$, which in turn, yields convergence in $C_{\mathrm{b}}\left(\mathbb{R}_{+}\right)^{d \times d}$ of the unique solutions $Z_{n}$ of the Volterra integral equations

$$
Z_{n}(x)=Z^{(0)}(x)-\left(V_{n} Z_{n}\right)(x), \quad x \geq 0,
$$

to the unique solution $Z$ of the integral equation (3.15). In particular,

$$
\lim _{n \rightarrow \infty} Z_{n}(0)=Z(0) \text { in } \mathbb{C}^{d \times d} \text { and hence } \lim _{n \rightarrow \infty} Y_{+, n}(0)=Y_{+}(0),
$$

where, similarly to $(3.21)$, we denote $Y_{+, n}(x)=e^{\lambda_{+}(Q) x} f_{\varepsilon}(x) Z_{n}(x)$. This proves continuity of the mapping (3.7).

Remark 3.3. This theorem holds under a weaker assumption than the exponential dichotomy hypothesis (cf.(2.15)). In fact, it is sufficient to require that $\varkappa_{+}(Q)<$ $\varkappa_{+}^{\prime}\left(I_{d}-Q\right)$ and $\varkappa_{+}(Q)<0$ only.

Remark 3.4. Replacing assumptions (3.5) and (3.6) by the hypothesis

$$
\|R\|_{\mathbb{C}^{d \times d}} \in L^{1}\left(\mathbb{R}_{+} ; e^{\beta x} d x\right) \text { with } \beta>-\lambda_{+}^{\prime}(Q),
$$

the matrix-valued Jost solution $Y_{+}$can be uniquely determined by solving the Volterra integral equation

$$
Y_{+}(x)=\Phi(x) Q-\int_{x}^{\infty} d x^{\prime} \Phi(x) \Phi\left(x^{\prime}\right)^{-1} R\left(x^{\prime}\right) Y_{+}\left(x^{\prime}\right), \quad x \geq 0 ;
$$

that is, in this case, the "modified" Volterra integral equation (3.14), (3.15) on $C_{\mathrm{b}}\left(\mathbb{R}_{+}\right)^{d \times d}$ is not needed. Indeed, assume $\beta>-\lambda_{+}^{\prime}(Q)$, choose $\varepsilon$ such that $0<$ $\varepsilon<\min \left\{\beta+\lambda_{+}^{\prime}(Q), \varkappa_{+}^{\prime}(I-Q)\right\}$, and use the inequalities $x^{\prime} \geq x \geq 0$ and $\lambda_{+}^{\prime}(Q)<$ $0<\varkappa_{+}^{\prime}(I-Q)$ to estimate the integral kernel of the integral operator in (3.30) as follows:

$$
\begin{aligned}
&\left\|\Phi(x) \Phi\left(x^{\prime}\right)^{-1} R\left(x^{\prime}\right)\right\|_{\mathbb{C}^{d \times d}} \leq\left(\|\Phi(x) Q\|_{\mathbb{C}^{d \times d}}\left\|Q \Phi\left(x^{\prime}\right)^{-1}\right\|_{\mathbb{C}^{d \times d}}\right. \\
&\left.+\left\|\Phi(x)(I-Q) \Phi\left(x^{\prime}\right)^{-1}\right\|_{\mathbb{C}^{d \times d}}\right)\left\|R\left(x^{\prime}\right)\right\|_{\mathbb{C}^{d \times d}} \\
& \leq\left(C(\varepsilon) e^{\left(-\lambda_{+}^{\prime}(Q)+\varepsilon\right) x^{\prime}}+C(\varepsilon) e^{\left(\varkappa_{+}^{\prime}(I-Q)-\varepsilon\right)\left(x-x^{\prime}\right)}\right)\left\|R\left(x^{\prime}\right)\right\|_{\mathbb{C}^{d \times d}} \\
& \leq 2 C(\varepsilon) e^{-\left(\lambda_{+}^{\prime}(Q)+\beta-\varepsilon\right) x^{\prime}} e^{\beta x^{\prime}}\left\|R\left(x^{\prime}\right)\right\|_{\mathbb{C}^{d \times d}} \leq 2 C(\varepsilon) e^{\beta x^{\prime}}\left\|R\left(x^{\prime}\right)\right\|_{\mathbb{C}^{d \times d}} .
\end{aligned}
$$

We recall that $\varkappa_{+}^{\prime}(Q) \leq \lambda_{+}^{\prime}(Q) \leq \lambda_{+}(Q)<0$. Condition (3.6) is formulated in terms of the difference $\lambda_{+}(Q)-\varkappa_{+}^{\prime}(Q)$ while condition (3.29) is formulated in terms of the quantity $-\lambda_{+}^{\prime}(Q)$. These two numbers, $\lambda_{+}(Q)-\varkappa_{+}^{\prime}(Q)$ and $-\lambda_{+}^{\prime}(Q)$ are fairly independent, and thus either of the two conditions, (3.6) and (3.29), can be better than the other. Thus, the existence of matrix-valued Jost solutions follows under the assumption $\beta>\min \left\{\lambda_{+}(Q)-\varkappa_{+}^{\prime}(Q),-\lambda_{+}^{\prime}(Q)\right\}$. 
Remark 3.5. Example 3.6 below shows that the statement of Theorem 3.2 is optimal in the sense that, under the exponential dichotomy hypothesis, the assumption in (3.6) on the exponential fall-off of the perturbation $R$ in general cannot be relaxed: Indeed, if condition (3.6) is violated, then the matrix-valued Jost solution may not exist. Example 3.6 also shows that if Definition 3.1 is modified to replace the strict inequality in (3.3) by the condition

$$
\limsup _{x \rightarrow \infty} \frac{\log \left\|Y_{+}(x)-\Phi(x) Q\right\|_{\mathbb{C}^{d \times d}}}{x} \leq \varkappa_{+}^{\prime}(Q),
$$

then the matrix-valued Jost solution in this modified sense is generally not unique. $\diamond$

Example 3.6. Consider equations (1.1) and (1.2) with

$$
A(x)=\left(\begin{array}{ccc}
-2 & 0 & 0 \\
0 & -1 & 0 \\
0 & 0 & 1
\end{array}\right) \text { and } R(x)=\left(\begin{array}{ccc}
0 & \chi_{\mathbb{R}_{+}}(x) e^{-x} \cos x & 0 \\
0 & 0 & 0 \\
0 & 0 & 0
\end{array}\right), \quad x \in \mathbb{R},
$$

where $\chi_{\mathbb{R}_{+}}(x)$ denotes the characteristic function of $\mathbb{R}_{+}=[0, \infty)$. The fundamental solution $\Phi$, the exponential dichotomy $Q$ on $\mathbb{R}$, and the bounded solution $\Phi Q$ of (1.1) on $\mathbb{R}_{+}$are given as follows:

$$
\Phi(x)=\left(\begin{array}{ccc}
e^{-2 x} & 0 & 0 \\
0 & e^{-x} & 0 \\
0 & 0 & e^{x}
\end{array}\right), \quad Q=\left(\begin{array}{ccc}
1 & 0 & 0 \\
0 & 1 & 0 \\
0 & 0 & 0
\end{array}\right), \quad \Phi(x) Q=\left(\begin{array}{ccc}
e^{-2 x} & 0 & 0 \\
0 & e^{-x} & 0 \\
0 & 0 & 0
\end{array}\right),
$$

The Bohl and Lyapunov exponents on $\mathbb{R}_{+}$associated with the dichotomy projection $Q$ are $\varkappa_{+}^{\prime}(Q)=\lambda_{+}^{\prime}(Q)=-2$ and $\varkappa_{+}(Q)=\lambda_{+}(Q)=-1$. Clearly, $\|R\|_{\mathbb{C}^{d \times d}} \in$ $L^{1}\left(\mathbb{R}_{+} ; e^{\beta x} d x\right)$ with $\beta=\lambda_{+}(Q)-\varkappa_{+}^{\prime}(Q)=1$, and hence condition (3.6) is violated. A direct computation shows that the perturbed equation (1.2) has two linearly independent bounded $\mathbb{C}^{d}$-valued solutions on $\mathbb{R}_{+}$

$$
y_{1}(x)=\left(\begin{array}{c}
e^{-2 x} \\
0 \\
0
\end{array}\right), \quad y_{2}(x)=\left(\begin{array}{c}
e^{-2 x} \sin x \\
e^{-x} \\
0
\end{array}\right), \quad x \geq 0,
$$

and thus any bounded $\mathbb{C}^{d}$-valued solution of $(1.2)$ on $\mathbb{R}_{+}$is a linear combination of these two. Hence, any bounded matrix-valued solution $Y$ of (1.2) on $\mathbb{R}_{+}$is necessarily of the form

$$
Y(x)=\left(\begin{array}{ccc}
e^{-2 x} & e^{-2 x}(\sin x+C) & 0 \\
0 & e^{-x} & 0 \\
0 & 0 & 0
\end{array}\right), \quad x \geq 0,
$$

where $C \in \mathbb{C}$ is an arbitrary constant. Thus, it satisfies

$$
\limsup _{x \rightarrow \infty} \frac{\log \|Y(x)-\Phi(x) Q\|_{\mathbb{C}^{d \times d}}}{x}=-2=\varkappa_{+}^{\prime}(Q) .
$$

This observation shows that the $d \times d$ matrix-valued Jost solution as introduced in Definition 3.1 does not exist if (3.6) fails, while the solutions satisfying (3.32) are not unique. 
Remark 3.7. In the proof of Theorem 3.2 we suggested a method of introducing the Jost solution by $Y_{+}(x)=e^{\mu x} Z(x), x \geq 0$, where $Z \in C_{\mathrm{b}}\left(\mathbb{R}_{+}\right)^{d \times d}$ is a solution of the " $\mu$-modified" Volterra integral equation

$$
Z(x)=e^{-\mu x} \Phi(x) Q-\int_{x}^{\infty} d x^{\prime} e^{-\mu\left(x-x^{\prime}\right)} \Phi(x) \Phi\left(x^{\prime}\right)^{-1} R\left(x^{\prime}\right) Z\left(x^{\prime}\right), \quad x \geq 0,
$$

for an appropriate choice of $\mu \in \mathbb{R}$. We stress that this method fails if the exponential fall-off hypothesis (3.6) is violated. Indeed, for the equations in Example 3.6, if $\mu<\lambda_{+}(Q)=-1$, the term $e^{-\mu x} \Phi(x) Q$ in the integral equation (3.38) is not a bounded function, while if $\mu \geq \varkappa_{+}(Q)=-1$, the Volterra integral operator is unbounded, both in the Banach space $C_{\mathrm{b}}\left(\mathbb{R}_{+}\right)^{d \times d}$ and in the Hilbert space $L^{2}\left(\mathbb{R}_{+}\right)^{d \times d}$.

Similar to the proof of Theorem 3.2 (replacing $\varkappa_{+}^{\prime}(Q)$ by $\varkappa_{-}\left(I_{d}-Q\right)$ and $\lambda_{+}(Q)$ by $\left.\lambda_{-}^{\prime}\left(I_{d}-Q\right)\right)$ one proves the following result for the negative half-line $\mathbb{R}_{-}$.

Theorem 3.8. Assume Hypothesis 2.8 and the condition

$$
\|R\|_{\mathbb{C}^{d \times d}} \in L^{1}\left(\mathbb{R}_{-} ; e^{\beta|x|} d x\right) \text { for some } \beta>\varkappa_{-}\left(I_{d}-Q\right)-\lambda_{-}^{\prime}\left(I_{d}-Q\right) .
$$

Then the perturbed differential equation in (1.2) has a unique matrix-valued Jost solution $Y_{-}(x), x \leq 0$. Moreover, the map

$$
\left\{\begin{aligned}
L^{1}\left(\mathbb{R}_{-} ; e^{\beta|x|}\right)^{d \times d} & \rightarrow \mathbb{C}^{d \times d} \\
R & \mapsto Y_{-}(0)
\end{aligned}\right.
$$

is continuous.

\section{The Perturbation Determinant}

In this section we employ results from [28], succinctly summarized in Appendix A, to express the infinite-dimensional (modified) Fredholm determinant of the integral operator $I+K$, given by (2.25), via a finite-dimensional determinant obtained by means of the matrix-valued Jost solutions introduced in Definition 3.1. This result is a generalization of the classical relation between a Fredholm determinant and the Jost function due to Jost and Pais [36] (see, also [46, Sect. 12.1.2]). As we will see in Section 9, the finite-dimensional determinant is related to the Evans function associated with (1.1) and (1.2). We recall Lemma 2.9, and also the general fact (see, e.g., [30, Ch. XIII], [31, Sect. IV.2], [65], [66, Ch. 9]) that if $K$ is any Hilbert-Schmidt operator, then the 2-modified Fredholm perturbation determinant $\operatorname{det}_{2}(I+K)$ is given by the formulas

$$
\operatorname{det}_{2}(I+K)=\operatorname{det}\left((I+K) e^{-K}\right)=\prod_{\lambda \in \sigma(K)}\left((1+\lambda) e^{-\lambda}\right),
$$

counting algebraic multiplicites of the eigenvalues of $K$. Moreover, if $K$ is a trace class operator, then

$$
\operatorname{det}_{2}(I+K)=e^{-\operatorname{tr}(K)} \operatorname{det}(I+K), \text { where } \operatorname{det}(I+K)=\prod_{\lambda \in \sigma(K)}(1+\lambda) .
$$

Throughout, we will use the following notation:

$$
\Theta=\int_{0}^{\infty} d x \operatorname{tr}_{\mathbb{C}^{d}}\left(\Phi(x) Q \Phi(x)^{-1} R(x)\right)-\int_{-\infty}^{0} d x \operatorname{tr}_{\mathbb{C}^{d}}\left(\Phi(x)\left(I_{d}-Q\right) \Phi(x)^{-1} R(x)\right) .
$$


Theorem 4.1. Assume Hypothesis 2.8 and the condition

$$
\|R\|_{\mathbb{C}^{d \times d}} \in L^{1}\left(\mathbb{R} ; e^{\beta|x|} d x\right)
$$

for some

$$
\beta>\max \left\{\lambda_{+}(Q)-\varkappa_{+}^{\prime}(Q), \varkappa_{-}\left(I_{d}-Q\right)-\lambda_{-}^{\prime}\left(I_{d}-Q\right)\right\} .
$$

Let $K$ be the integral operator on $L^{2}(\mathbb{R})^{d}$ whose integral kernel is given by (2.25). Then the 2-modified perturbation determinant $\operatorname{det}_{2}(I+K)$ admits the representation

$$
\operatorname{det}_{2}(I+K)=e^{\Theta} \operatorname{det}_{\mathbb{C}^{d}}\left(Y_{+}(0)+Y_{-}(0)\right),
$$

where $\Theta$ is defined in (4.3), and $Y_{ \pm}$are the matrix-valued Jost solutions on $\mathbb{R}_{ \pm}$, respectively.

Proof. Assume temporarily that

$$
\operatorname{supp}(R) \text { is compact. }
$$

Then $\|R\|_{\mathbb{C}^{d \times d}} \in L^{1}\left(\mathbb{R} ; e^{\beta|x|} d x\right)$ for any real $\beta$. In particular, by Remark 3.4 and its obvious $\mathbb{R}_{-}$-analog, the $d \times d$ matrix-valued Jost solutions $Y_{ \pm}$are the unique bounded solutions of the following Volterra integral equations on $\mathbb{R}_{ \pm}$,

$$
\begin{aligned}
& Y_{+}(x)=\Phi(x) Q-\int_{x}^{\infty} d x^{\prime} \Phi(x) \Phi\left(x^{\prime}\right)^{-1} R\left(x^{\prime}\right) Y_{+}\left(x^{\prime}\right), \quad x \geq 0, \\
& Y_{-}(x)=\Phi(x)\left(I_{d}-Q\right)+\int_{-\infty}^{x} d x^{\prime} \Phi(x) \Phi\left(x^{\prime}\right)^{-1} R\left(x^{\prime}\right) Y_{-}\left(x^{\prime}\right), \quad x \leq 0 .
\end{aligned}
$$

We note that $Y_{+}(x)=Y_{+}(x) Q$ and $Y_{-}(x)=Y_{-}(x)\left(I_{d}-Q\right)$ by uniqueness of the solutions. Moreover, by (4.7) we have $Y_{+}(x)=\Phi(x) Q$ for $x \geq n$ and $Y_{-}(x)=$ $\Phi(x)\left(I_{d}-Q\right)$ for $x \leq-n$ for sufficiently large $n \in \mathbb{N}$.

Treating matrices as operators on respective spaces, we introduce the following notations for $x \in \mathbb{R}$,

$$
\begin{aligned}
& f_{1}(x)=R_{r}(x) \Phi(x) Q: \operatorname{ran}(Q) \rightarrow \mathbb{C}^{d}, \\
& f_{2}(x)=R_{r}(x) \Phi(x)\left(I_{d}-Q\right): \operatorname{ker}(Q) \rightarrow \mathbb{C}^{d}, \\
& g_{1}(x)=Q \Phi(x)^{-1} R_{\ell}(x): \mathbb{C}^{d} \rightarrow \operatorname{ran}(Q), \\
& g_{2}(x)=-\left(I_{d}-Q\right) \Phi(x)^{-1} R_{\ell}(x): \mathbb{C}^{d} \rightarrow \operatorname{ker}(Q) .
\end{aligned}
$$

In addition, let $d_{1}=\operatorname{dim}(\operatorname{ran}(Q))$ and $d_{2}=\operatorname{dim}(\operatorname{ker}(Q))$, and denote

$$
H\left(x, x^{\prime}\right)=f_{1}(x) g_{1}\left(x^{\prime}\right)-f_{2}(x) g_{2}\left(x^{\prime}\right)=R_{r}(x) \Phi(x) \Phi\left(x^{\prime}\right)^{-1} R_{\ell}\left(x^{\prime}\right), \quad x, x^{\prime} \in \mathbb{R} .
$$

We note that the functions $f_{j}$ and $g_{j}, j=1,2$, are compactly supported on $\mathbb{R}$ due to (4.7), and thus assumption (A.1) holds. Hence, the results recorded in Appendix $\mathrm{A}$ are at our disposal. Next, we introduce the Volterra integral equations

$$
\begin{array}{ll}
\hat{f}_{1}(x)=f_{1}(x)-\int_{x}^{\infty} d x^{\prime} H\left(x, x^{\prime}\right) \hat{f}_{1}\left(x^{\prime}\right), & x \in \mathbb{R}, \\
\hat{f}_{2}(x)=f_{2}(x)+\int_{-\infty}^{x} d x^{\prime} H\left(x, x^{\prime}\right) \hat{f}_{2}\left(x^{\prime}\right), & x \in \mathbb{R},
\end{array}
$$

and let $\hat{f}_{1}(x): \operatorname{ran}(Q) \rightarrow \mathbb{C}^{d}$ and $\hat{f}_{2}(x): \operatorname{ker}(Q) \rightarrow \mathbb{C}^{d}$ be the unique solutions of (4.10) that satisfy $\hat{f}_{j} \in L^{2}(\mathbb{R})^{d \times d_{j}}, j=1,2$ (cf. Appendix A). The $d \times d$ matrix 
$U(x), x \in \mathbb{R}$, defined in (A.9), written as a block-operator with respect to the decomposition $\mathbb{C}^{d}=\operatorname{ran}(Q) \dot{+} \operatorname{ker}(Q)$, then reads as follows:

$$
U(x)=\left(\begin{array}{cc}
I_{d_{1}}-\int_{x}^{\infty} d x^{\prime} g_{1}\left(x^{\prime}\right) \hat{f}_{1}\left(x^{\prime}\right) & \int_{-\infty}^{x} d x^{\prime} g_{1}\left(x^{\prime}\right) \hat{f}_{2}\left(x^{\prime}\right) \\
\int_{x}^{\infty} d x^{\prime} g_{2}\left(x^{\prime}\right) \hat{f}_{1}\left(x^{\prime}\right) & I_{d_{2}}-\int_{-\infty}^{x} d x^{\prime} g_{2}\left(x^{\prime}\right) \hat{f}_{2}\left(x^{\prime}\right)
\end{array}\right) .
$$

Writing $R(x)=R_{\ell}(x) R_{r}(x)$, and multiplying (4.8) by $R_{r}(x)$ from the left, we arrive at (4.10) and observe that

$$
\begin{array}{ll}
\hat{f}_{1}(x)=\left.R_{r}(x) Y_{+}(x)\right|_{\operatorname{ran}(Q)}, & x \geq 0, \\
\hat{f}_{2}(x)=\left.R_{r}(x) Y_{-}(x)\right|_{\operatorname{ker}(Q)}, & x \leq 0 .
\end{array}
$$

Setting $x=0$ in (4.8), using (4.9) and (4.12), and writing the matrix $Y_{+}(0)+Y_{-}(0)$ as a block-operator with respect to the decomposition $\mathbb{C}^{d}=\operatorname{ran}(Q) \dot{+} \operatorname{ker}(Q)$, we conclude from (4.11) at $x=0$ that $U(0)=Y_{+}(0)+Y_{-}(0)$. Since the integral kernel $K\left(x, x^{\prime}\right)$ in $(2.25)$ can be represented as

$$
K\left(x, x^{\prime}\right)=\left\{\begin{array}{ll}
-f_{1}(x) g_{1}\left(x^{\prime}\right), & x \geq x^{\prime}, \\
-f_{2}(x) g_{2}\left(x^{\prime}\right), & x<x^{\prime},
\end{array} \quad x, x^{\prime} \in \mathbb{R},\right.
$$

Theorem A.1 (ii) proves (4.6) for compactly supported perturbations. Specifically, one can use (A.13) with $x_{0}=0$ and then apply some elementary properties of matrix traces.

To remove the compact support assumption on the matrix-valued function $R$ one proceeds as follows. Given $n \in \mathbb{N}$, we introduce the truncations

$$
R_{n}(x)= \begin{cases}R(x), & |x| \leq n, \\ 0, & |x|>n\end{cases}
$$

Let $R_{n}(x)=V_{n}(x)\left|R_{n}(x)\right|, x \in \mathbb{R}$, be the polar decompositions of $R_{n}(x)$, denote $R_{\ell, n}(x)=V_{n}(x)\left|R_{n}(x)\right|^{1 / 2}$ and $R_{r, n}(x)=\left|R_{n}(x)\right|^{1 / 2}$, and introduce the integral kernels of Hilbert-Schmidt integral operators $K_{n}$ on $L^{2}(\mathbb{R})^{d}$ by

$$
K_{n}\left(x, x^{\prime}\right)=\left\{\begin{array}{ll}
-R_{r, n}(x) \Phi(x) Q \Phi\left(x^{\prime}\right)^{-1} R_{\ell, n}\left(x^{\prime}\right), & x \geq x^{\prime}, \\
R_{r, n}(x) \Phi(x)\left(I_{d}-Q\right) \Phi\left(x^{\prime}\right)^{-1} R_{\ell, n}\left(x^{\prime}\right), & x<x^{\prime},
\end{array} \quad x, x^{\prime} \in \mathbb{R} .\right.
$$

Since the support of $R_{n}$ is compact, one infers by the first step of the proof that

$$
\operatorname{det}_{2}\left(I+K_{n}\right)=e^{\Theta_{n}} \operatorname{det}_{\mathbb{C}^{d}}\left(Y_{+, n}(0)+Y_{-, n}(0)\right), \quad n \in \mathbb{N},
$$

where

$$
\begin{aligned}
\Theta_{n}= & \int_{0}^{\infty} d x \operatorname{tr}_{\mathbb{C}^{d}}\left(\Phi(x) Q \Phi(x)^{-1} R_{n}(x)\right) \\
& -\int_{-\infty}^{0} d x \operatorname{tr}_{\mathbb{C}^{d}}\left(\Phi(x)\left(I_{d}-Q\right) \Phi(x)^{-1} R_{n}(x)\right)
\end{aligned}
$$

and $Y_{ \pm, n}$ are the matrix-valued Jost solutions of the truncated perturbed equation $y^{\prime}=\left(A+R_{n}\right) y$ on $\mathbb{R}_{ \pm}$. Since $R_{n}$ converges to $R$ in $L^{1}(\mathbb{R})^{d \times d}$ as $n \rightarrow \infty$, one concludes that

$$
\lim _{n \rightarrow \infty} \Theta_{n}=\Theta
$$


where $\Theta$ is given by (4.3). Using the estimate in the proof of Lemma 2.9, one checks that the operators $K_{n}$ converge to the operator $K$ in Hilbert-Schmidt norm, and hence (see, e.g., [30, Ch. IX], [66, Ch. 9])

$$
\lim _{n \rightarrow \infty} \operatorname{det}_{2}\left(I+K_{n}\right)=\operatorname{det}_{2}(I+K) .
$$

Finally, since the sequence $R_{n}$ converges to $R$ in $L^{1}\left(\mathbb{R} ; e^{\beta|x|} d x\right)^{d \times d}$ as $n \rightarrow \infty$, applying assertions (3.7) and (3.40) of Theorems 3.2 and 3.8, we obtain

$$
\lim _{n \rightarrow \infty} Y_{ \pm, n}(0)=Y_{ \pm}(0) .
$$

Combining (4.18)-(4.20) then completes the proof.

Remark 4.2. The following heuristic argument may be helpful in understanding the role of the factor $e^{\Theta}$ in (4.6) and shows that the appearance of this factor is quite natural. First, we observe that the integral kernel (2.25) of the operator $K$ is generally discontinuous on the diagonal $x=x^{\prime}$. Accordingly, the definition of $\Theta$ in (4.3) can be re-written as follows:

$$
-\Theta=\int_{0}^{\infty} d x \operatorname{tr}_{\mathbb{C}^{d}}(K(x+0, x))+\int_{-\infty}^{0} d x \operatorname{tr}_{\mathbb{C}^{d}}(K(x-0, x)) .
$$

Heuristically, the right-hand side of (4.21) can be viewed as a "regularized integral trace" of the operator $K$. Next, we assume in addition that $K$ is a trace class operator (this requires quite a stretch of imagination!), and that $\operatorname{tr}(K)$ equals the integral trace $-\Theta$,

$$
\operatorname{tr}(K)=-\Theta
$$

Then formally applying (4.2) and (4.6) yields

$$
\operatorname{det}_{2}(I+K)=e^{-\operatorname{tr}(K)} \operatorname{det}(I+K)=e^{\Theta} \operatorname{det}_{\mathbb{C}^{d}}\left(Y_{+}(0)+Y_{-}(0)\right)
$$

and hence formally,

$$
\operatorname{det}(I+K)=\operatorname{det}_{\mathbb{C}^{d}}\left(Y_{+}(0)+Y_{-}(0)\right) .
$$

As we will see in Section 9, the determinant $\operatorname{det}_{\mathbb{C}^{d}}\left(Y_{+}(0)+Y_{-}(0)\right)$ is in fact the Evans function for the equations (1.1) and (1.2).

Remark 4.3. The proof of Theorem 4.1 shows that the 2-modified determinant of the operator $I+K$ can be computed by the formula

$$
\operatorname{det}_{2}(I+K)=e^{\Theta} \lim _{n \rightarrow \infty} \operatorname{det}_{\mathbb{C}^{d}}\left(Y_{+, n}(0)+Y_{-, n}(0)\right)
$$

under the weaker assumption $\|R\|_{\mathbb{C}^{d \times d}} \in L^{1}(\mathbb{R})$ as opposed to (4.4) and (4.5). Here $Y_{ \pm, n}$ are the unique matrix-valued Jost solutions of the Volterra integral equations

$$
\begin{aligned}
& Y_{+, n}(x)=\Phi(x) Q-\int_{x}^{\infty} d x^{\prime} \Phi(x) \Phi\left(x^{\prime}\right)^{-1} R_{n}\left(x^{\prime}\right) Y_{+, n}\left(x^{\prime}\right), \quad x \geq 0, \\
& Y_{-, n}(x)=\Phi(x)\left(I_{d}-Q\right)+\int_{-\infty}^{x} d x^{\prime} \Phi(x) \Phi\left(x^{\prime}\right)^{-1} R_{n}\left(x^{\prime}\right) Y_{-, n}\left(x^{\prime}\right), \quad x \leq 0,
\end{aligned}
$$

and $R_{n}$ is the truncated perturbation (4.14). We also have the following identities:

$$
Y_{+, n}(0)=Y_{+, n}(0) Q, \quad Y_{-, n}(0)=Y_{-, n}(0)\left(I_{d}-Q\right),
$$

since the solutions $Y_{ \pm, n}$ are unique. 
Remark 4.4. The exponential fall-off assumption (4.5) has been imposed in Theorem 4.1 to make sure that the matrix-valued Jost solutions $Y_{ \pm}$exist and are unique (cf. Remark 3.5). Moreover, under this assumption, one uses continuity of the mapping (3.7) to conclude that

$$
\begin{aligned}
\lim _{n \rightarrow \infty} \operatorname{det}_{\mathbb{C}^{d}}\left(Y_{+, n}(0)+Y_{-, n}(0)\right) & =\operatorname{det}_{\mathbb{C}^{d}}\left(\lim _{n \rightarrow \infty}\left(Y_{+, n}(0)+Y_{-, n}(0)\right)\right) \\
& =\operatorname{det}_{\mathbb{C}^{d}}\left(Y_{+}(0)+Y_{-}(0)\right) .
\end{aligned}
$$

Example 3.6 shows that the exponential fall-off assumptions (4.5) are indeed needed to interchange the limit $n \rightarrow \infty$ and the determinant in (4.28). In fact, in this example one immediately verifies that the Jost solutions $Y_{ \pm, n}$ associated with the truncated perturbation $R_{n}$ are given by

$$
\begin{aligned}
& Y_{+, n}(x)=\left\{\begin{array}{ccc}
\left(\begin{array}{ccc}
e^{-2 x} & \left.e^{-2 x}[\sin (x)-\sin (n)]\right) & 0 \\
0 & e^{-x} & 0 \\
0 & 0 & 0
\end{array}\right), & 0 \leq x \leq n, \\
\left(\begin{array}{ccc}
e^{-2 x} & 0 & 0 \\
0 & e^{-x} & 0 \\
0 & 0 & 0
\end{array}\right), & x>n,
\end{array}\right. \\
& Y_{-, n}(x)=\left(\begin{array}{ccc}
0 & 0 & 0 \\
0 & 0 & 0 \\
0 & 0 & e^{x}
\end{array}\right), \quad x \leq 0 .
\end{aligned}
$$

Clearly, the sequence of the $3 \times 3$ matrices $Y_{+, n}(0)$ does not converge as $n \rightarrow \infty$, while $\lim _{n \rightarrow \infty} \operatorname{det}_{\mathbb{C}^{d}}\left(Y_{+, n}(0)+Y_{-, n}(0)\right)$ does exist since $\operatorname{det}_{\mathbb{C}^{d}}\left(Y_{+, n}(0)+Y_{-, n}(0)\right)=1$ for all $n \in \mathbb{N}$. Consequently, (4.28) fails.

\section{Sub-exponential Weights and the Jost Function}

As we have seen in Section 3, the exponential fall-off hypotheses (3.6) and (3.39) on the perturbation $R$ cannot be relaxed in general because the unperturbed equation might have at least one of the following two properties: First, the upper Lyapunov exponent associated with the dichotomy projection $Q$ may not coincide with the lower Bohl exponent and then, necessarily, the corresponding Bohl interval is of positive width; and, second, the estimate (2.5) used in the definition of the lower Bohl exponent $\varkappa^{\prime}(Q)$ may not hold for $\varkappa=\varkappa^{\prime}(Q)$ but only for $\varkappa=\varkappa^{\prime}(Q)-\varepsilon$ for any $\varepsilon>0$. This results in the de facto presence of an exponential weight factor $f_{\varepsilon}\left(\left|x-x^{\prime}\right|\right)=C(\varepsilon) e^{\varepsilon\left|x-x^{\prime}\right|}$ on the right-hand side of (2.5); cf. also (3.11) and the effect of this on the proof of Theorem 3.2. In this section we restrict our attention to the class of unperturbed equations (1.1) that do not have either of these properties. That is, we consider the case when each of the Bohl segments associated with the dichotomy projections $Q$ and $\left(I_{d}-Q\right)$ degenerates into a single point and, in addition, we assume that the exponential weight factors in the estimates such as (2.5) are replaced by sub-exponential weight factors induced by a given monotone weight function $f$.

We introduce a weight function, $f$, which, by definition, is a nondecreasing function $f: \mathbb{R}_{+} \rightarrow \mathbb{R}_{+}$, satisfying the following conditions:

$$
\limsup _{x \rightarrow \infty} \frac{\log (f(x))}{x}=0, \quad f(0) \geq 1 .
$$


Hypothesis 5.1. Assume Hypothesis 2.8. Suppose that the Bohl segments on $\mathbb{R}$ associated with the projections $Q$ and $\left(I_{d}-Q\right)$ have zero width, that is,

$$
\varkappa^{\prime}(Q)=\varkappa(Q)<0<\varkappa^{\prime}\left(I_{d}-Q\right)=\varkappa\left(I_{d}-Q\right) .
$$

Given a weight function $f$, we asssume, in addition, that the following estimates hold for all $x, x^{\prime} \in \mathbb{R}$ :

$$
\begin{gathered}
\left\|\Phi(x) Q \Phi\left(x^{\prime}\right)^{-1}\right\|_{\mathbb{C}^{d \times d}} \leq e^{\varkappa(Q)\left(x-x^{\prime}\right)} f\left(\left|x-x^{\prime}\right|\right), \quad 0 \leq x \leq x^{\prime}, \\
\left\|\Phi(x)\left(I_{d}-Q\right) \Phi\left(x^{\prime}\right)^{-1}\right\|_{\mathbb{C}^{d \times d}} \leq e^{\varkappa\left(I_{d}-Q\right)\left(x-x^{\prime}\right)} f\left(\left|x-x^{\prime}\right|\right), \quad 0 \geq x \geq x^{\prime} .
\end{gathered}
$$

Given Hypothesis 5.1 we now introduce the matrix-valued Jost solutions as solutions of certain "modified" Volterra integral equations (cf. (3.14)-(3.15) and (4.8)).

Definition 5.2. Assume Hypothesis 5.1 and suppose

$$
\|R\|_{\mathbb{C}^{d \times d}} \in L^{1}\left(\mathbb{R} ; f^{2}(|x|) d x\right) .
$$

Then matrix-valued solutions $Y_{ \pm}$of (1.2) on $\mathbb{R}_{ \pm}$are called matrix-valued Jost solutions of (1.2) if

$$
\begin{aligned}
& Y_{+}(x)=f(|x|) e^{\varkappa(Q) x} Z_{+}(x), \quad x \geq 0, \\
& Y_{-}(x)=f(|x|) e^{\varkappa\left(I_{d}-Q\right) x} Z_{-}(x), \quad x \leq 0,
\end{aligned}
$$

where $Z_{+}(x), x \geq 0$, and $Z_{-}(x), x \leq 0$, are the unique bounded solutions of the Volterra integral equations

$$
\begin{aligned}
& Z_{+}(x)=e^{-\varkappa(Q) x} f(x)^{-1} \Phi(x) Q \\
& \quad-\int_{x}^{\infty} d x^{\prime} e^{-\varkappa(Q)\left(x-x^{\prime}\right)} f(x)^{-1} f\left(x^{\prime}\right) \Phi(x) \Phi\left(x^{\prime}\right)^{-1} R\left(x^{\prime}\right) Z_{+}\left(x^{\prime}\right), \quad x \geq 0 \\
& Z_{-}(x)=e^{-\varkappa\left(I_{d}-Q\right) x} f(|x|)^{-1} \Phi(x)(I-Q) \\
& \quad+\int_{-\infty}^{x} d x^{\prime} e^{-\varkappa\left(I_{d}-Q\right)\left(x-x^{\prime}\right)} f(|x|)^{-1} f\left(\left|x^{\prime}\right|\right) \Phi(x) \Phi\left(x^{\prime}\right)^{-1} R\left(x^{\prime}\right) Z_{-}\left(x^{\prime}\right), \quad x \leq 0
\end{aligned}
$$

on $\mathbb{R}_{+}$and $\mathbb{R}_{-}$.

Almost literally repeating the proof of Theorems 3.2 and 3.8 (replacing $f_{\varepsilon}$ by $f$ ), one concludes that the matrix-valued Jost solutions $Y_{ \pm}$in the sense of Definition 5.2 satisfy the following asymptotic relations (cf. (3.22)):

$$
\begin{aligned}
& e^{-\varkappa(Q) x}\left\|Y_{+}(x)-\Phi(x) Q\right\|_{\mathbb{C}^{d \times d}} \underset{x \rightarrow \infty}{=} o(1), \\
& e^{-\varkappa\left(I_{d}-Q\right) x}\left\|Y_{-}(x)-\Phi(x)\left(I_{d}-Q\right)\right\|_{\mathbb{C}^{d \times d}} \underset{x \rightarrow-\infty}{=} o(1) .
\end{aligned}
$$

Moreover, one obtains the following version of Theorem 4.1.

Theorem 5.3. Assume Hypothesis 5.1, suppose that

$$
\|R\|_{\mathbb{C}^{d \times d}} \in L^{1}\left(\mathbb{R} ; f^{2}(|x|) d x\right),
$$

and let the integral kernel of the operator $K$ be given by (2.25). Then the 2-modified perturbation determinant $\operatorname{det}_{2}(I+K)$ admits the representation

$$
\operatorname{det}_{2}(I+K)=e^{\Theta} \operatorname{det}_{\mathbb{C}^{d}}\left(Y_{+}(0)+Y_{-}(0)\right),
$$

where $\Theta$ is defined in (4.3) and $Y_{ \pm}$are the matrix-valued Jost solutions on $\mathbb{R}_{ \pm}$ introduced in Definition 5.2. 
In the remaining part of this section we will apply Theorem 5.3 to study the Schrödinger equation (3.1) with an integrable potential,

$$
V \in L^{1}(\mathbb{R}) \text {. }
$$

We consider equations (1.1) and (1.2) with

$$
A(k)=\left(\begin{array}{cc}
0 & 1 \\
-k^{2} & 0
\end{array}\right), \quad R(x)=\left(\begin{array}{cc}
0 & 0 \\
V(x) & 0
\end{array}\right), \quad x \in \mathbb{R} .
$$

To avoid confusion we emphasize that $A$ is an $x$-independent function of the parameter $k$ in (3.1). Clearly, (1.2), with $A=A(k)$ and $R$ as in (5.11), is the first-order system corresponding to the Schrödinger equation (3.1). We note that $\sigma(A(k))=$ $\{i k,-i k\}$, where, without loss of generality, we choose $k$ so that $\operatorname{Im}(k) \geq 0$. Since we intend to apply Theorem 5.3, we have to make sure that Hypothesis 2.8 is satisfied. In particular, the unperturbed equation with the $x$-independent coefficient $A(k)$ must have an exponential dichotomy on $\mathbb{R}$. This is equivalent to the requirement $\sigma(A(k)) \cap i \mathbb{R}=\emptyset$ or, taking into account the choice of $k$, to the inequality $\operatorname{Im}(k)>0$, which we will assume to hold in the remaining part of this section.

Let $Q(k)$ be the spectral projection for $A(k)$ so that $\sigma\left(\left.A(k)\right|_{\operatorname{ran}(Q(k))}\right)=\{i k\}$. We remark that

$$
\varkappa^{\prime}(Q(k))=\varkappa(Q(k))=\operatorname{Re}(i k) \text { and } \varkappa^{\prime}\left(I_{d}-Q(k)\right)=\varkappa\left(I_{d}-Q(k)\right)=\operatorname{Re}(-i k) .
$$

Next, we introduce the sub-exponential weight function by

$$
f(x)=c, \quad x \in \mathbb{R}_{+},
$$

for an appropriate constant $c \geq 1$. Since the eigenvalues $\pm i k$ of $A(k)$ are simple, the estimate (5.3) with $\Phi(k, x)=e^{x A(k)}, x \in \mathbb{R}$, holds, and thus Hypothesis 5.1 is satisfied. The matrix-valued Jost solutions $Y_{ \pm}(k, \cdot)$ in the sense of Definition 5.2 are the unique solutions of the Volterra integral equations

$$
\begin{aligned}
& Y_{+}(k, x)=e^{x A(k)} Q(k)-\int_{x}^{\infty} d x^{\prime} e^{\left(x-x^{\prime}\right) A(k)} R\left(x^{\prime}\right) Y_{+}\left(k, x^{\prime}\right), \quad x \geq 0, \\
& Y_{-}(k, x)=e^{x A(k)}\left(I_{d}-Q(k)\right)+\int_{-\infty}^{x} d x^{\prime} e^{\left(x-x^{\prime}\right) A(k)} R\left(x^{\prime}\right) Y_{-}\left(k, x^{\prime}\right), \quad x \leq 0,
\end{aligned}
$$

such that the matrix-valued functions $Z_{ \pm}(k, x)=e^{\mp i k x} Y_{ \pm}(k, x), x \in \mathbb{R}_{ \pm}$, are bounded. Since $V \in L^{1}(\mathbb{R})$, the conclusion of Theorem 5.3 holds with $Y_{ \pm}(k, \cdot)$ given by (5.14).

Next, we will relate the finite-dimensional determinant in (5.9) and the classical Jost function (see, e.g., [6, Ch. XVII], [46, Sect. 12.1] for the latter). First, we recall some well-known notions from scattering theory (see [28] for a detailed bibliography). The Jost solutions, $u_{ \pm}(k, \cdot)$, of the Schrödinger equation (3.1) are defined as solutions of the Volterra integral equations

$$
\begin{array}{r}
u_{ \pm}(k, x)=e^{ \pm i k x}-\int_{x}^{ \pm \infty} d x^{\prime} k^{-1} \sin \left(k\left(x-x^{\prime}\right)\right) V\left(x^{\prime}\right) u_{ \pm}\left(k, x^{\prime}\right), \\
\operatorname{Im}(k)>0, x \in \mathbb{R} .
\end{array}
$$

The Jost function, $\mathcal{J}=\mathcal{J}(k)$ is defined by

$$
\mathcal{J}(k)=\frac{1}{2 i k} W\left(u_{-}(k, \cdot), u_{+}(k, \cdot)\right), \quad \operatorname{Im}(k)>0,
$$


where $W(u(x), v(x))=u(x) v^{\prime}(x)-u^{\prime}(x) v(x)$ is the Wronskian of $u$ and $v$ with $u, v \in C^{1}(\mathbb{R})$. For $k>0, \mathcal{J}(\mathrm{k})$ is the reciprocal of the transmission coefficient (see, e.g., formula (XVII.1.36) in [6]). We note that $\mathcal{J}(k)$ is independent of $x \in \mathbb{R}$ since $\operatorname{tr}_{\mathbb{C}^{2}}(A(k))=0$.

Next, we consider the factorization $V=V_{\ell} V_{r}$ with

$$
V_{\ell}(x)=|V(x)|^{1 / 2}, \quad V_{r}(x)=|V(x)|^{1 / 2} \exp (i \arg (V(x)), \quad x \in \mathbb{R} .
$$

To make the connection with $(2.23)$, we remark that

$$
R_{\ell}(x)=\left(\begin{array}{cc}
0 & 0 \\
V_{\ell}(x) & 0
\end{array}\right), \quad R_{r}(x)=\left(\begin{array}{cc}
V_{r}(x) & 0 \\
0 & 0
\end{array}\right), \quad x \in \mathbb{R} .
$$

Finally, we introduce the integral operator, $L(k)$ on $L^{2}(\mathbb{R})$ with integral kernel

$$
L\left(k, x, x^{\prime}\right)=\frac{i}{2 k} V_{r}(x) e^{i k\left|x-x^{\prime}\right|} V_{\ell}\left(x^{\prime}\right), \quad \operatorname{Im}(k)>0, x, x^{\prime} \in \mathbb{R} .
$$

The following corollary of Theorem 5.3 recovers a well-known relation between the Fredholm determinant of the operator $I+L(k)$ and the Jost function originally due to Jost and Pais [36] (see also [44], [45], [46, Sect. 12.1.1] and, specifically, [28, Theorem 4.7]). More importantly, it shows that the classical Jost function coincides with the finite-dimensional determinant $\operatorname{det}_{\mathbb{C}^{d}}\left(Y_{+}(k, 0)+Y_{-}(k, 0)\right)$ in $(5.9)$ obtained by means of the matrix-valued Jost solutions introduced in Definition 5.2. As we will see in Section 9, this determinant is, in fact, the Evans function associated with equations (1.1) and (1.2).

Theorem 5.4. Assume that the potential of the Schrödinger equation (3.1) satisfies $V \in L^{1}(\mathbb{R})$, and fix $k \in \mathbb{C}$ such that $\operatorname{Im}(k)>0$. Let $K(k)$ be the integral operator on $L^{2}(\mathbb{R})^{2}$ with the integral kernel given by $(2.25)$, where $\Phi(k, x)=e^{x A(k)}, x \in \mathbb{R}$, and $A(k)$ is defined in (5.11), and $R_{\ell}$ and $R_{r}$ are defined by (5.18). Let $L(k)$ be the integral operator on $L^{2}(\mathbb{R})$ with the integral kernel given by $(5.19)$ and $\mathcal{J}(k)$ be the Jost function of (3.1). Finally, let $Y_{ \pm}(k, \cdot)$ be the matrix-valued Jost solutions (5.14) on $\mathbb{R}_{ \pm}$for (1.1) and (1.2) with $A(k)$ and $R$ given by (5.11). Then the following assertions hold:

(i) $\mathcal{J}(k)=\operatorname{det}_{\mathbb{C}^{2}}\left(Y_{+}(k, 0)+Y_{-}(k, 0)\right)$.

(ii) The 2-modified Fredholm determinant admits the following representation:

$$
\operatorname{det}_{2}(I+K(k))=\operatorname{det}_{2}(I+L(k))=e^{\Theta(k)} \mathcal{J}(k),
$$

where

$$
\Theta(k)=\frac{1}{2 i k} \int_{\mathbb{R}} d x V(x) .
$$

Proof. It is convenient to diagonalize $A(k)$ and $e^{x A(k)}, x \in \mathbb{R}$. To this effect we introduce the matrices

$$
\begin{aligned}
T(k) & =\left(\begin{array}{cc}
1 & 1 \\
i k & -i k
\end{array}\right), \quad T(k)^{-1}=\frac{1}{2 i k}\left(\begin{array}{cc}
i k & 1 \\
i k & -1
\end{array}\right), \\
\widetilde{A}(k) & =T(k)^{-1} A(k) T(k)=\left(\begin{array}{cc}
i k & 0 \\
0 & -i k
\end{array}\right), \\
\widetilde{Q} & =\left(\begin{array}{ll}
1 & 0 \\
0 & 0
\end{array}\right), \quad S(k)=\frac{1}{2 i k}\left(\begin{array}{cc}
1 & 1 \\
-1 & -1
\end{array}\right), \\
Q(k) & =T(k) \widetilde{Q} T(k)^{-1}=\frac{1}{2}\left(\begin{array}{cc}
1 & (i k)^{-1} \\
i k & 1
\end{array}\right) .
\end{aligned}
$$


We note that $\widetilde{Q}$ is the spectral projection for $\widetilde{A}(k)$ so that

$$
\sigma\left(\left.\widetilde{A}(k)\right|_{\operatorname{ran}(\widetilde{Q})}\right)=\sigma\left(\left.A(k)\right|_{\operatorname{ran}(Q(k))}\right)=\{i k\} .
$$

Passing to the matrix-valued functions $\widetilde{Y}_{ \pm}(k, x)=T(k)^{-1} Y_{ \pm}(k, x) T(k), x \in \mathbb{R}_{ \pm}$, in (5.14), we obtain the integral equations

$$
\begin{aligned}
& \widetilde{Y}_{+}(k, x)=e^{x \widetilde{A}(k)} \widetilde{Q}-\int_{x}^{\infty} d x^{\prime} e^{\left(x-x^{\prime}\right) \widetilde{A}(k)} V\left(x^{\prime}\right) S(k) \widetilde{Y}_{+}\left(k, x^{\prime}\right), \quad x \geq 0, \\
& \widetilde{Y}_{-}(k, x)=e^{x \widetilde{A}}(I-\widetilde{Q})+\int_{-\infty}^{x} d x^{\prime} e^{\left(x-x^{\prime}\right) \widetilde{A}(k)} V\left(x^{\prime}\right) S(k) \widetilde{Y}_{-}\left(k, x^{\prime}\right), \quad x \leq 0 .
\end{aligned}
$$

The property $\widetilde{Y}_{+}(k, \cdot)=\widetilde{Y}_{+}(k, \cdot) \widetilde{Q}$ and $(5.25)$ imply that the second column of the $2 \times 2$ matrix $\widetilde{Y}_{+}(k, \cdot)$ is equal to zero, while $\widetilde{Y}_{-}(k, \cdot)=\widetilde{Y}_{-}(k, \cdot)\left(I_{2}-\widetilde{Q}\right)$ and (5.26) imply that the first column of $\widetilde{Y}_{-}(k, \cdot)$ is equal to zero. Thus, in the matrix equations (5.25) and (5.26) we can separately consider the first and the second column, respectively. Let $\widetilde{y}_{+}(k, \cdot)$ denote the first column of $\widetilde{Y}_{+}(k, \cdot)$ and $\widetilde{y}_{-}(k, \cdot)$ denote the second column of $\widetilde{Y}_{-}(k, \cdot)$. Passing in $(5.25)$ and $(5.26)$ to the $\mathbb{C}^{2}$ valued functions $y_{ \pm}(k, x)=T(k) \widetilde{y}_{ \pm}(k, x), x \in \mathbb{R}_{ \pm}$, we observe ${ }^{2}$ that they satisfy the following $\mathbb{C}^{2}$-valued integral equations:

$$
\begin{array}{r}
y_{+}(k, x)=e^{i k x}(1 \quad i k)^{\top}-\int_{x}^{\infty} d x^{\prime} V\left(x^{\prime}\right) T(k) e^{\left(x-x^{\prime}\right) \tilde{A}(k)} S(k) T(k)^{-1} y_{+}\left(k, x^{\prime}\right), \\
y_{-}(k, x)=e^{-i k x}(1-i k)^{\top}+\int_{-\infty}^{x} d x^{\prime} V\left(x^{\prime}\right) T(k) e^{\left(x-x^{\prime}\right) \widetilde{A}(k)} S(k) T(k)^{-1} y_{-}\left(k, x^{\prime}\right), \\
x \leq 0 .
\end{array}
$$

A direct calculation using (5.22) and (5.23) shows that

$$
\begin{gathered}
T(k) e^{\left(x-x^{\prime}\right) \widetilde{A}(k)} S(k) T(k)^{-1}=\left(\begin{array}{cc}
k^{-1} \sin \left(k\left(x-x^{\prime}\right)\right) & 0 \\
\cos \left(k\left(x-x^{\prime}\right)\right) & 0
\end{array}\right), \\
\operatorname{Im}(k)>0, x, x^{\prime} \in \mathbb{R} .
\end{gathered}
$$

Since $Y_{ \pm}(k, \cdot)$ are matrix-valued solutions of $(1.2)$, it follows that $y_{ \pm}(k, \cdot)$ are $\mathbb{C}^{2}$ valued solutions of $(1.2)$. Next, we denote by $u_{ \pm}(k, \cdot)$ the top entry of the $2 \times 1$ vector $y_{ \pm}(k, \cdot)$. Differentiating the first components in the $2 \times 1$ vector integral equations in (5.27), we observe that $y_{ \pm}(k, x)=\left(u_{ \pm}(k, x) \quad u_{ \pm}^{\prime}(k, x)\right)^{\top}, x \in \mathbb{R}_{ \pm}$, and thus $u_{ \pm}(k, \cdot)$ are (scalar-valued) solutions of the Schrödinger equation (3.1). Moreover, it follows from (5.27) that $u_{ \pm}(k, \cdot)$ satisfy $(5.15)$, that is, $u_{ \pm}(k, \cdot)$ are the traditional Jost solutions of (3.1). This proves assertion $(i)$ since

$$
\begin{aligned}
& \operatorname{det}_{\mathbb{C}^{2}}\left(Y_{+}(k, 0)+Y_{-}(k, 0)\right)=\operatorname{det}_{\mathbb{C}^{2}}\left(\widetilde{Y}_{+}(k, 0)+\widetilde{Y}_{-}(k, 0)\right) \\
& \quad=\operatorname{det}_{\mathbb{C}^{2}}\left(\left(\widetilde{y}_{+}(k, 0) \widetilde{y}_{-}(k, 0)\right)\right)=\operatorname{det}_{\mathbb{C}^{2}}\left(T(k)^{-1}\left(y_{+}(k, 0) y_{-}(k, 0)\right)\right) \\
& \quad=\frac{-1}{2 i k} W\left(u_{+}(k), u_{-}(k)\right)=\mathcal{J}(k) .
\end{aligned}
$$

Finally, we turn to the proof of assertion (ii). A direct computation in (4.3) using (5.11) and the formula for $Q(k)$ in (5.23), verifies the formula for $\Theta(k)$ in (5.21). Using (2.25) and (5.18), and with the help of the diagonalization described in (5.22)

\footnotetext{
${ }^{2}$ We use $\top$ for transposition so that $(a b)^{\top}$ is a $(2 \times 1)$ column vector.
} 
and (5.23), one computes that the matrix-valued integral kernel $K\left(k, x, x^{\prime}\right)$ in $(2.25)$ and the scalar-valued integral kernel $L\left(k, x, x^{\prime}\right)$ in (5.19) are related by the formula

$$
K\left(k, x, x^{\prime}\right)=L\left(k, x, x^{\prime}\right) \widetilde{Q}, \quad x, x^{\prime} \in \mathbb{R},
$$

where $\widetilde{Q}$ is the projection in (5.23). This implies $\operatorname{det}_{2}(I+K(k))=\operatorname{det}_{2}(I+L(k))$. Thus, relation (5.9) and assertion $(i)$ yield assertion $(i i)$.

While we assumed $\operatorname{Im}(k)>0$ throughout this section, we note that continuity of $K(k)$ and $L(k)$ with respect to $k, \operatorname{Im}(k) \geq 0, k \neq 0$, in the Hilbert-Schmidt norm, permits one to extend the results of Theorem $5.4(i),(i i)$ to all $\operatorname{Im}(k) \geq 0, k \neq 0$, by continuity.

\section{Generalized Matrix-Valued Jost Solutions}

In this section we start the discussion of a generalization of the matrix-valued Jost solutions introduced in Definition 3.1, and prove an extension of Theorem 3.2 assuming that the dichotomy projection $Q$ admits further exponential splitting. As we will see later on, the generalized matrix-valued Jost solutions will allow us to relax the exponential fall-off hypothesis on the perturbation imposed in (4.5). We recall from Remark 3.5 that these hypotheses are optimal, provided one merely assumes the existence of an exponential dichotomy $Q$ with no further splitting. To simplify the exposition in this section, we will only consider the case where the dichotomy projection $Q$ admits a splitting of order two and postpone the general case of the exponential splitting of arbitrary order until Section 7.

Hypothesis 6.1. Assume Hypothesis 2.8. In addition, assume that the dichotomy projection $Q$ can be represented in the form $Q=Q_{1}+Q_{2}$, where $Q_{1}$ and $Q_{2}$ are projections which are uniformly conjugated by $\Phi$ on $\mathbb{R}$ such that the Bohl segment $\left[\varkappa^{\prime}\left(Q_{1}\right), \varkappa\left(Q_{1}\right)\right]$ lies strictly below $\left[\varkappa^{\prime}\left(Q_{2}\right), \varkappa\left(Q_{2}\right)\right]$, that is,

$$
\varkappa^{\prime}\left(Q_{1}\right) \leq \varkappa\left(Q_{1}\right)<\varkappa^{\prime}\left(Q_{2}\right) \leq \varkappa\left(Q_{2}\right)<0<\varkappa^{\prime}\left(I_{d}-Q\right) \leq \varkappa\left(I_{d}-Q\right) .
$$

We will consider the generalized $d \times d$ matrix-valued Jost solutions on $\mathbb{R}_{+}$(cf. Definition 3.1 ), the case of $\mathbb{R}_{-}$can be treated similarly.

Definition 6.2. Assume $R \in L_{\text {loc }}^{1}(\mathbb{R})^{d \times d}$ and Hypothesis 6.1. Then $d \times d$ matrixvalued solutions $Y_{+}^{(1)}$ and $Y_{+}^{(2)}$ of the perturbed differential equation (1.2) on $\mathbb{R}_{+}$ are called generalized matrix-valued Jost solutions associated with the projections $Q_{1}$ and $Q_{2}$ if

$$
\limsup _{x \rightarrow \infty} \frac{\log \left\|Y_{+}^{(j)}(x)-\Phi(x) Q_{j}\right\|_{\mathbb{C}^{d \times d}}}{x}<\varkappa_{+}^{\prime}\left(Q_{j}\right)
$$

and

$$
Y_{+}^{(j)}(0)=Y_{+}^{(j)}(0) Q_{j}, \quad j=1,2 .
$$

We emphasize that the additional technical requirement in (6.3) can always be satisfied as soon as (6.2) holds for some solutions $Y_{+}^{(j)}, j=1,2$. Indeed, if the solutions $Y_{+}^{(j)}$ satisfy $(6.2)$, then $Y_{+}^{(j)} Q_{j}, j=1,2$, are solutions of the perturbed differential equation satisfying both (6.2) and (6.3). 
Remark 6.3. We note that the generalized matrix-valued Jost solution $Y_{+}^{(2)}$ is not unique. Indeed, if $Y_{+}^{(2)}$ is a solution satisfying (6.2) for $j=2$, and $Y_{+}$is any solution of (1.2) whose upper Lyapunov exponent on $\mathbb{R}_{+}$belongs to the Bohl segment $\left[\varkappa_{+}^{\prime}\left(Q_{1}\right), \varkappa_{+}\left(Q_{1}\right)\right]$, then the solution $Y_{+}^{(2)}+Y_{+}$again satisfies (6.2) for $j=2$ since

$$
\begin{aligned}
& \limsup _{x \rightarrow \infty} \frac{\log \left\|Y_{+}^{(2)}(x)+Y_{+}(x)-\Phi(x) Q_{2}\right\|_{\mathbb{C}^{d \times d}}}{x} \\
& \quad \leq \max \left\{\limsup _{x \rightarrow \infty} \frac{\log \left\|Y_{+}^{(2)}(x)-\Phi(x) Q_{2}\right\|_{\mathbb{C}^{d \times d}}}{x}, \varkappa_{+}\left(Q_{1}\right)\right\}<\varkappa_{+}^{\prime}\left(Q_{2}\right) .
\end{aligned}
$$

We start with the following elementary fact.

Lemma 6.4. Let $\alpha>0$ and assume that $0 \leq p \in L^{1}\left(\mathbb{R}_{+}\right)$. Then,

$$
\lim _{x \rightarrow \infty} e^{-\alpha x} \int_{0}^{x} d x^{\prime} e^{\alpha x^{\prime}} p\left(x^{\prime}\right)=0 .
$$

Proof. Assuming mes $(\{x \geq 0 \mid p(x)>0\})>0$ and introducing $q(x)=\int_{0}^{x} d x^{\prime} p\left(x^{\prime}\right)$, $x \geq 0$, an integration by parts yields

$$
e^{-\alpha x} \int_{0}^{x} d x^{\prime} e^{\alpha x^{\prime}} p\left(x^{\prime}\right)=q(x)-\alpha e^{-\alpha x} \int_{0}^{x} d x^{\prime} e^{\alpha x^{\prime}} q\left(x^{\prime}\right), \quad x \geq 0 .
$$

Since $p$ is nonnegative and integrable on $\mathbb{R}_{+}, \lim _{x \rightarrow \infty} q(x)$ is positive, and the integral $\int_{0}^{x} d x^{\prime} e^{\alpha x^{\prime}} q\left(x^{\prime}\right)$ diverges. Using l'Hôpital's rule one obtains assertion (6.5).

The following result ensures the existence of the generalized matrix-valued Jost solutions on $\mathbb{R}_{+}$introduced in Definition 6.2 under much weaker exponential fall-off assumptions on the perturbation than (3.5) and (3.6) (we recall that the requirement (3.5) is optimal if we want to deal with the matrix-valued solutions introduced in Definition 3.1 only).

Theorem 6.5. Assume Hypothesis 6.1 and the condition

$$
\|R\|_{\mathbb{C}^{d \times d}} \in L^{1}\left(\mathbb{R}_{+} ; e^{\beta x} d x\right)
$$

with some

$$
\beta>\max _{j=1,2}\left\{\lambda_{+}\left(Q_{j}\right)-\varkappa_{+}^{\prime}\left(Q_{j}\right)\right\}
$$

Then the perturbed differential equation (1.2) has generalized matrix-valued Jost solutions $Y_{+}^{(j)}, j=1,2$.

Proof. Existence and uniqueness of the generalized Jost solution $Y_{+}^{(1)}$ on $\mathbb{R}_{+}$associated with the projection $Q_{1}$ follows from Theorem 3.2 and Remark 3.3; to check condition (6.3) for $j=1$ we note that $Y_{+}^{(1)}(x)=Y_{+}^{(1)}(x) Q, x \geq 0$, since the solution $Y_{+}^{(1)}$ is unique.

By hypothesis and the definition of the lower Bohl exponents (cf. (2.5)), for any $\varepsilon>0$ there exists a positive constant $C(\varepsilon)>0$ such that

$$
\begin{aligned}
& \left\|\Phi(x)\left(I-Q_{1}\right) \Phi\left(x^{\prime}\right)^{-1}\right\|_{\mathbb{C}^{d \times d}} \\
& \quad \leq\left\|\Phi(x) Q_{2} \Phi\left(x^{\prime}\right)^{-1}\right\|_{\mathbb{C}^{d \times d}}+\left\|\Phi(x)\left(I_{d}-Q\right) \Phi\left(x^{\prime}\right)^{-1}\right\|_{\mathbb{C}^{d \times d}}
\end{aligned}
$$




$$
\leq\left(e^{\left(\varkappa_{+}^{\prime}\left(Q_{2}\right)\right)\left(x-x^{\prime}\right)}+e^{\left(\varkappa_{+}^{\prime}\left(I_{d}-Q\right)\right)\left(x-x^{\prime}\right)}\right) f_{\varepsilon}\left(\left|x-x^{\prime}\right|\right), \quad 0 \leq x \leq x^{\prime},
$$

where $f_{\varepsilon}(x)=C(\varepsilon) e^{\varepsilon|x|}$. Since $\varkappa_{+}^{\prime}\left(Q_{2}\right)<\varkappa_{+}^{\prime}\left(I_{d}-Q\right)$, we infer the estimate

$$
\left\|\Phi(x)\left(I-Q_{1}\right) \Phi\left(x^{\prime}\right)^{-1}\right\|_{\mathbb{C}^{d \times d}} \leq 2 e^{\left(\varkappa_{+}^{\prime}\left(Q_{2}\right)\right)\left(x-x^{\prime}\right)} f_{\varepsilon}\left(\left|x-x^{\prime}\right|\right), \quad 0 \leq x \leq x^{\prime} .
$$

By the definition of the upper Bohl exponent, one may also assume that

$$
\left\|\Phi(x) Q_{1} \Phi\left(x^{\prime}\right)^{-1}\right\|_{\mathbb{C}^{d \times d}} \leq 2 e^{\left(\varkappa+\left(Q_{1}\right)\right)\left(x-x^{\prime}\right)} f_{\varepsilon}\left(\left|x-x^{\prime}\right|\right), \quad 0 \leq x^{\prime} \leq x .
$$

Taking $\varepsilon>0$ such that $0<2 \varepsilon<\beta-\left(\lambda_{+}\left(Q_{2}\right)-\varkappa_{+}^{\prime}\left(Q_{2}\right)\right)$, and introducing the function

$$
p(x)=e^{\left(\lambda_{+}\left(Q_{2}\right)-\varkappa_{+}^{\prime}\left(Q_{2}\right)\right) x} f_{\varepsilon}^{2}(x)\|R(x)\|_{\mathbb{C}^{d \times d}}, \quad x \geq 0,
$$

one observes that $0 \leq p \in L^{1}\left(\mathbb{R}_{+}\right)$by assumptions (6.7) and (6.8). For brevity we denote $\mu=\lambda_{+}\left(Q_{2}\right), \alpha=\varkappa_{+}^{\prime}\left(Q_{2}\right)-\varkappa_{+}\left(Q_{1}\right)$, and, for any $\tau \geq 0$, we introduce the integral kernel

$$
F_{\mu}^{\tau}\left(x, x^{\prime}\right)=e^{-\mu\left(x-x^{\prime}\right)} \frac{f_{\varepsilon}\left(x^{\prime}\right)}{f_{\varepsilon}(x)} \begin{cases}-\Phi(x)\left(I-Q_{1}\right) \Phi\left(x^{\prime}\right)^{-1} R\left(x^{\prime}\right), & \tau \leq x<x^{\prime}, \\ \Phi(x) Q_{1} \Phi\left(x^{\prime}\right)^{-1} R\left(x^{\prime}\right), & \tau \leq x^{\prime} \leq x .\end{cases}
$$

Using (6.10) and (6.11) and the inequalities $\varkappa_{+}^{\prime}\left(Q_{2}\right)-\lambda_{+}\left(Q_{2}\right) \leq 0$ and $\alpha>0$, one derives the following estimate:

$$
\begin{aligned}
& \left\|F_{\mu}^{\tau}\left(x, x^{\prime}\right)\right\|_{\mathbb{C}^{d \times d}} \leq 2 f_{\varepsilon}(x)^{-1} f_{\varepsilon}^{2}\left(x^{\prime}\right)\left\|R\left(x^{\prime}\right)\right\|_{\mathbb{C}^{d \times d}} \\
& \times \begin{cases}e^{\left(\varkappa_{+}^{\prime}\left(Q_{2}\right)-\lambda_{+}\left(Q_{2}\right)\right)\left(x-x^{\prime}\right)}, & \tau \leq x<x^{\prime}, \\
e^{\left(\varkappa_{+}\left(Q_{1}\right)-\lambda_{+}\left(Q_{2}\right)\right)\left(x-x^{\prime}\right)}, & \tau \leq x^{\prime} \leq x,\end{cases} \\
& =2 f_{\varepsilon}(x)^{-1} e^{\left(\varkappa_{+}^{\prime}\left(Q_{2}\right)-\lambda_{+}\left(Q_{2}\right)\right) x} p\left(x^{\prime}\right) \begin{cases}1, & \tau \leq x<x^{\prime}, \\
e^{-\alpha\left(x-x^{\prime}\right)}, & \tau \leq x^{\prime} \leq x,\end{cases} \\
& \leq 2 f_{\varepsilon}(x)^{-1} p\left(x^{\prime}\right), \quad x, x^{\prime} \geq \tau .
\end{aligned}
$$

On the space $C_{\mathrm{b}}([\tau, \infty))^{d \times d}$ we define the integral operator $F_{\mu}^{\tau}$ by

$$
\left(F_{\mu}^{\tau} Z\right)(x)=\int_{\tau}^{\infty} d x^{\prime} F_{\mu}^{\tau}\left(x, x^{\prime}\right) Z\left(x^{\prime}\right), \quad x \geq \tau,
$$

and consider the corresponding Fredholm-type integral equation

$$
Z(x)=Z^{(0)}(x)-\left(F_{\mu}^{\tau} Z\right)(x), \quad x \geq \tau,
$$

where $Z^{(0)}$ is defined by

$$
Z^{(0)}(x)=e^{-\mu x} f_{\varepsilon}^{-1}(x) \Phi(x) Q_{2}, \quad x \geq \tau .
$$

Since $f_{\varepsilon} \geq 1$ and $p \in L^{1}\left(\mathbb{R}_{+}\right)$, the estimate in (6.15) shows that the integral operator $F_{\mu}^{\tau}$ is a contraction on $C_{\mathrm{b}}([\tau, \infty))^{d \times d}$ for $\tau \geq 0$ sufficiently large. Since the exponential decay of $\left\|\Phi Q_{2}\right\|_{\mathbb{C}^{d \times d}}$ is controlled by $\mu$, we see that $Z^{(0)} \in C_{\mathrm{b}}([\tau, \infty))^{d \times d}$. Thus, the integral equation (6.17) has a unique solution $Z \in C_{\mathrm{b}}([\tau, \infty))^{d \times d}$ that can be obtained by the iteration process

$$
Z(x)=\sum_{j=1}^{\infty} Z^{(j)}(x), \quad Z^{(j)}(x)=\left(F_{\mu}^{\tau} Z^{(j-1)}\right)(x), \quad j \in \mathbb{N}, x \geq \tau .
$$

Clearly, by uniqueness of the solution,

$$
Z(x)=Z(x) Q_{2}, \quad x \geq \tau .
$$


A straightforward computation shows that the function

$$
Y_{+}^{(2)}(x)=e^{\mu x} f_{\varepsilon}(x) Z(x), \quad x \geq \tau,
$$

yields a solution of the perturbed differential equation in (1.2). Moreover, from (6.17) and (6.14) it also follows that

$$
\begin{gathered}
f_{\varepsilon}(x)\left\|Z(x)-Z^{(0)}(x)\right\|_{\mathbb{C}^{d \times d}} \leq 2 e^{\left(\varkappa_{+}^{\prime}\left(Q_{2}\right)-\lambda_{+}\left(Q_{2}\right)\right) x}\left(\|p\|_{L^{1}([x, \infty))}\right. \\
\left.\quad+e^{-\alpha x} \int_{0}^{x} d x^{\prime} p\left(x^{\prime}\right) e^{\alpha x^{\prime}}\right)\|Z\|_{C_{\mathrm{b}}([\tau, \infty))^{d \times d}}, \quad x \geq \tau .
\end{gathered}
$$

Using $p \in L^{1}\left(\mathbb{R}_{+}\right)$and Lemma 6.4, (6.22) implies the asymptotic relation

$$
e^{-\varkappa_{+}^{\prime}\left(Q_{2}\right) x}\left\|Y_{+}^{(2)}(x)-\Phi(x) Q_{2}\right\|_{\mathbb{C}^{d \times d}} \underset{x \rightarrow \infty}{=} o(1) .
$$

Moreover, using (6.7) and (6.8) again, choose $\delta \in(0, \alpha)$ sufficiently small such that the function $p_{\delta}(x)=e^{\delta x} p(x), x \geq 0$, is integrable on $\mathbb{R}_{+}$. Then (6.22) implies

$$
\begin{gathered}
f_{\varepsilon}(x)\left\|Z(x)-Z^{(0)}(x)\right\|_{\mathbb{C}^{d \times d}} \leq 2 e^{-\delta x} e^{\left(\varkappa_{+}^{\prime}\left(Q_{2}\right)-\lambda_{+}\left(Q_{2}\right)\right) x}\left(\left\|p_{\delta}\right\|_{L^{1}([x, \infty))}\right. \\
\left.+e^{-(\alpha-\delta) x} \int_{0}^{x} d x^{\prime} p_{\delta}\left(x^{\prime}\right) e^{(\alpha-\delta) x^{\prime}}\right)\|Z\|_{C_{\mathrm{b}}([\tau, \infty)) d \times d}, \quad x \geq \tau .
\end{gathered}
$$

Using $p_{\delta} \in L^{1}\left(\mathbb{R}_{+}\right)$and Lemma 6.4 again, one obtains the asymptotic relation

$$
e^{-\varkappa_{+}^{\prime}\left(Q_{2}\right) x}\left\|Y_{+}^{(2)}(x)-\Phi(x) Q_{2}\right\|_{\mathbb{C}^{d \times d}} \underset{x \rightarrow \infty}{=} o\left(e^{-\delta x}\right) .
$$

Finally, the matrix-valued solution $Y_{+}^{(2)}$ can be uniquely extended to the interval $[0, \tau)$ by solving the initial value problem

$$
Y_{+}^{(2)^{\prime}}(x)=(A(x)+B(x)) Y_{+}^{(2)}(x), \quad x \geq \tau, \quad Y_{+}^{(2)}(\tau)=e^{\mu \tau} f_{\varepsilon}(\tau) Z(\tau) .
$$

In addition, from (6.25) one derives (6.2), and from (6.20) it follows that $Y_{+}^{(2)}(0)=$ $Y_{+}^{(2)}(0) Q_{2}$, completing the proof.

In order to discuss uniqueness properties of the generalized $d \times d$ matrix-valued Jost solution $Y_{+}^{(2)}$ up to lower-order terms (cf. Remark 6.3), we recall that if $Q$ is the (unique) exponential dichotomy projection for the unperturbed equation (1.1) on $\mathbb{R}$, then equation (1.1) also has an exponential dichotomy on $\mathbb{R}_{+}$with the same dichotomy projection $Q$. However, the corresponding Bohl segment on $\mathbb{R}_{+}$may be strictly smaller than that on $\mathbb{R}$, that is, $\left[\varkappa_{+}^{\prime}(Q), \varkappa_{+}(Q)\right] \subset\left[\varkappa^{\prime}(Q), \varkappa(Q)\right]$, see $(2.10)$. By Lemma 2.13, the perturbed differential equation with $\|R\|_{\mathbb{C}^{d \times d}} \in L^{1}\left(\mathbb{R}_{+}\right)$will also have an exponential dichotomy on $\mathbb{R}_{+}$with a dichotomy projection $P$ having the same Bohl exponents as the unperturbed differential equation for the dichotomy projection $Q$. We emphasize that the dichotomy projection $P$ is not unique: Only the subspace $\operatorname{ran}(P)$ is determined uniquely since it consists precisely of those initial data $y_{0}$ such that the $\mathbb{C}^{d}$-valued solutions of the initial value problem

$$
y^{\prime}(x)=(A(x)+R(x)) y(x), \quad x \geq 0, \quad y(0)=y_{0},
$$

are bounded on $\mathbb{R}_{+}$. Moreover, by Lemma 2.14, if $Q=Q_{1}+Q_{2}$ is an exponential splitting for (1.1) on $\mathbb{R}$ (and therefore on $\mathbb{R}_{+}$), then the dichotomy projection $P$ for (1.2) on $\mathbb{R}_{+}$also admits an exponential splitting $P=P_{1}+P_{2}$ with the same Bohl segments, that is, $\left[\varkappa_{+}^{\prime}\left(P_{j}\right), \varkappa_{+}\left(P_{j}\right)\right]=\left[\varkappa_{+}^{\prime}\left(Q_{j}\right), \varkappa_{+}\left(Q_{j}\right)\right], j=1,2$. The projection 
$P_{1}$ is not uniquely determined but its range is, since $\operatorname{ran}\left(P_{1}\right)$ consists of precisely those initial data $y_{0}$ such that the Lyapunov exponent of the $\mathbb{C}^{d}$-valued solution of (6.27) satisfies $\lambda_{+}(y) \leq \varkappa_{+}\left(P_{1}\right)<\varkappa_{+}^{\prime}\left(P_{2}\right)=\varkappa_{+}^{\prime}\left(Q_{2}\right)$ (cf. Remark 2.7). Thus, in view of Theorem 6.5 and Remark 6.3 , we have proved the following uniqueness result:

Corollary 6.6. Assume the hypotheses of Theorem 6.5. Then:

(i) The generalized matrix-valued Jost solution $Y_{+}^{(1)}$ associated with the projection $Q_{1}$ is unique.

(ii If $Y_{+}^{(2)}$ and $\widetilde{Y}_{+}^{(2)}$ are any two generalized matrix-valued Jost solutions associated with the projection $Q_{2}$ then $\operatorname{ran}\left(Y_{+}^{(2)}(0)-\widetilde{Y}_{+}^{(2)}(0)\right) \subseteq \operatorname{ran}\left(P_{1}\right)$.

\section{Exponential Splitting of Arbitrary Order}

In this section we consider the generalized matrix-valued Jost solutions of (1.2) in the general case where the unperturbed equation (1.1) has an exponential splitting of arbitrary order on $\mathbb{R}$.

Hypothesis 7.1. Assume Hypothesis 2.8 with $d \geq 2$. Suppose, in addition, that for some $d^{\prime}, 2 \leq d^{\prime} \leq d$ and $k_{0}, 1 \leq k_{0} \leq d^{\prime}-1$, the dichotomy projection $Q$ on $\mathbb{R}$ for (1.1) admits an exponential splitting $Q=\sum_{j=1}^{k_{0}} Q_{j}$ of order $k_{0}$, and the projection $\left(I_{d}-Q\right)$ admits an exponential splitting $I-Q=\sum_{j=k_{0}+1}^{d^{\prime}} Q_{j}$ of order $d^{\prime}-k_{0}$, where the projections $Q_{j}, j=1,2, \ldots, d^{\prime}$, are uniformly conjugated by $\Phi$ on $\mathbb{R}$ and the corresponding disjoint Bohl segments $\left[\varkappa^{\prime}\left(Q_{j}\right), \varkappa\left(Q_{j}\right)\right]$ are ordered as follows:

$$
\varkappa^{\prime}\left(Q_{j}\right) \leq \varkappa\left(Q_{j}\right)<\varkappa^{\prime}\left(Q_{j+1}\right) \leq \varkappa\left(Q_{j+1}\right), \quad 1 \leq j \leq d^{\prime}-1 .
$$

Definition 7.2. Assume $R \in L_{\mathrm{loc}}^{1}(\mathbb{R})^{d \times d}$ and Hypothesis 7.1. Then $d \times d$ matrixvalued solutions $Y_{+}^{(j)}, j=1, \ldots, k_{0}$, on $\mathbb{R}_{+}$and $Y_{-}^{(j)}, j=k_{0}+1, \ldots, d^{\prime}$, on $\mathbb{R}_{-}$of the differential equation

$$
Y^{\prime}(x)=(A(x)+R(x)) Y(x), \quad x \in \mathbb{R}_{ \pm},
$$

are called generalized matrix-valued Jost solutions associated with the exponential splitting $\left\{Q_{j}\right\}_{j=1}^{d^{\prime}}$ if

$$
\begin{aligned}
& \limsup _{x \rightarrow \infty} \frac{\log \left\|Y_{+}^{(j)}(x)-\Phi(x) Q_{j}\right\|_{\mathbb{C}^{d \times d}}}{x}<\varkappa_{+}^{\prime}\left(Q_{j}\right), \\
& Y_{+}^{(j)}(0)=Y_{+}^{(j)}(0) Q_{j}, \quad j=1,2, \ldots, k_{0}
\end{aligned}
$$

and

$$
\begin{aligned}
& \liminf _{x \rightarrow-\infty} \frac{\log \left\|Y_{-}^{(j)}(x)-\Phi(x) Q_{j}\right\|_{\mathbb{C}^{d \times d}}}{x}>\varkappa_{-}\left(Q_{j}\right), \\
& Y_{-}^{(j)}(0)=Y_{-}^{(j)}(0) Q_{j}, \quad j=k_{0}+1, \ldots, d^{\prime} .
\end{aligned}
$$

Assuming Hypothesis 7.1, and referring to Lemmas 2.13-2.14, let

$$
P_{+}=P_{1}+P_{2}+\cdots+P_{k_{0}}
$$

be the exponential splitting of order $k_{0}$ of the dichotomy projection $P_{+}$for (1.2) on $\mathbb{R}_{+}$such that

$$
\left[\varkappa_{+}^{\prime}\left(P_{j}\right), \varkappa_{+}\left(P_{j}\right)\right]=\left[\varkappa_{+}^{\prime}\left(Q_{j}\right), \varkappa_{+}\left(Q_{j}\right)\right], \quad j=1, \ldots, k_{0} .
$$


Similarly, let

$$
P_{-}=P_{k_{0}+1}+P_{k_{0}+2}+\cdots+P_{d^{\prime}}
$$

be the exponential splitting of order $\left(d^{\prime}-k_{0}\right)$ of the dichotomy projection $P_{-}$for (1.2) on $\mathbb{R}_{-}$such that

$$
\left[\varkappa_{-}^{\prime}\left(P_{j}\right), \varkappa_{-}\left(P_{j}\right)\right]=\left[\varkappa_{-}^{\prime}\left(Q_{j}\right), \varkappa_{-}\left(Q_{j}\right)\right], \quad j=k_{0}+1, \ldots, d^{\prime} .
$$

Our next result shows that the generalized matrix-valued Jost solutions exist and are unique up to lower exponential order terms.

Theorem 7.3. Assume Hypothesis 7.1 and the condition

$$
\|R\|_{\mathbb{C}^{d \times d}} \in L^{1}\left(\mathbb{R} ; e^{\beta|x|} d x\right)
$$

with some

$$
\beta>\max \left\{\max _{1 \leq j \leq k_{0}}\left\{\lambda_{+}\left(Q_{j}\right)-\varkappa_{+}^{\prime}\left(Q_{j}\right)\right\}, \max _{k_{0}+1 \leq j \leq d^{\prime}}\left\{\varkappa_{-}\left(Q_{j}\right)-\lambda_{-}^{\prime}\left(Q_{j}\right)\right\}\right\} .
$$

Then the following conclusion holds:

(i) The perturbed equation (1.2) has generalized matrix-valued Jost solutions in the sense of Definition 7.2, $Y_{+}^{(j)}, j=1,2, \ldots, k_{0}$, on $\mathbb{R}_{+}$and $Y_{-}^{(j)}, j=k_{0}+1, \ldots, d^{\prime}$, on $\mathbb{R}_{-}$associated with the exponential splitting $\left\{Q_{j}\right\}_{j=1}^{d^{\prime}}$.

For any set of generalized matrix-valued Jost solutions in the sense of Definition 7.2, the following assertions hold:

(ii) The generalized matrix-valued Jost solutions $Y_{+}^{(1)}$ and $Y_{-}^{\left(d^{\prime}\right)}$, associated with the projections $Q_{1}$ and $Q_{d^{\prime}}$, are uniquely determined and satisfy

$$
\operatorname{ran}\left(Y_{+}^{(1)}(0)\right) \subseteq \operatorname{ran}\left(P_{1}\right), \quad \operatorname{ran}\left(Y_{-}^{\left(d^{\prime}\right)}(0)\right) \subseteq \operatorname{ran}\left(P_{d^{\prime}}\right) .
$$

(iii) The generalized matrix-valued Jost solutions $Y_{+}^{(j)}, j=2, \ldots, k_{0}$, and $Y_{-}^{(j)}$, $j=k_{0}+1, \ldots, d^{\prime}-1$, satisfy

$$
\begin{aligned}
& \operatorname{ran}\left(Y_{+}^{(j)}(0)\right) \backslash\{0\} \subseteq \operatorname{ran}\left(\sum_{k=1}^{j} P_{k}\right) \backslash \operatorname{ran}\left(\sum_{k=1}^{j-1} P_{k}\right), \quad j=2, \ldots, k_{0}, \\
& \operatorname{ran}\left(Y_{-}^{(j)}(0)\right) \backslash\{0\} \subseteq \operatorname{ran}\left(\sum_{k=j}^{d^{\prime}} P_{k}\right) \backslash \operatorname{ran}\left(\sum_{k=j+1}^{d^{\prime}} P_{k}\right), \quad j=k_{0}+1, \ldots, d^{\prime}-1 .
\end{aligned}
$$

(iv) If $Y_{+}^{(j)}$ and $\widetilde{Y}_{+}^{(j)}$ are any two generalized matrix-valued Jost solutions associated with the projections $Q_{j}$ for $j=2, \ldots, k_{0}$ and $Y_{-}^{(j)}$ and $\widetilde{Y}_{-}^{(j)}$ are any two generalized Jost solutions associated with the projection $Q_{j}$ for $j=k_{0}+1, \ldots, d^{\prime}-1$, then

$$
\begin{aligned}
& \operatorname{ran}\left(Y_{+}^{(j)}(0)-\tilde{Y}_{+}^{(j)}(0)\right) \subseteq \operatorname{ran}\left(\sum_{k=1}^{j-1} P_{k}\right), \quad j=2, \ldots, k_{0}, \\
& \operatorname{ran}\left(Y_{-}^{(j)}(0)-\widetilde{Y}_{-}^{(j)}(0)\right) \subseteq \operatorname{ran}\left(\sum_{k=j+1}^{d^{\prime}} P_{k}\right), \quad j=k_{0}+1, \ldots, d^{\prime}-1 .
\end{aligned}
$$

Proof. We will provide a sketch of the proof for the case of $\mathbb{R}_{+}$referring for details to the proof of Theorem 6.5. The case of $\mathbb{R}_{-}$is treated similarly. 
To prove $(i)$ and the uniqueness statement in $(i i)$, we fix $j \in\left\{1, \ldots, k_{0}\right\}$ and denote $\mu_{j}=\lambda_{+}\left(Q_{j}\right)+\varepsilon, j=1, \ldots, k_{0}$, for some $\varepsilon>0$. In addition, for some $\tau \geq 0$, we introduce the integral operator $F_{\mu_{j}}^{\tau, j}$ with integral kernel

$$
F_{\mu_{j}}^{\tau, j}\left(x, x^{\prime}\right)=e^{-\mu_{j}\left(x-x^{\prime}\right)} \begin{cases}-\Phi(x)\left[\sum_{k=j}^{d^{\prime}} Q_{k}\right] \Phi\left(x^{\prime}\right)^{-1} R\left(x^{\prime}\right), & \tau \leq x<x^{\prime}, \\ \Phi(x)\left[\sum_{k=1}^{j-1} Q_{k}\right] \Phi\left(x^{\prime}\right)^{-1} R\left(x^{\prime}\right), & \tau \leq x^{\prime} \leq x,\end{cases}
$$

on the Banach space $C_{\mathrm{b}}([\tau, \infty))^{d \times d}$. Repeating the arguments in the proof of Theorem 6.5 under hypothesis (7.12), one concludes that the operator $F_{\mu_{j}}^{\tau, j}$ is a contraction on $C_{\mathrm{b}}([\tau, \infty))^{d \times d}$, provided $\tau$ is large enough and $\varepsilon$ is sufficiently small. Thus, for each such $\tau$, the Fredholm-type integral equation

$$
Z^{(j)}(x)=Z_{0}^{(j)}(x)-\left(F_{\mu_{j}}^{\tau, j} Z^{(j)}\right)(x), \quad x \geq \tau,
$$

where $Z_{0}^{(j)}$ is defined by

$$
Z_{0}^{(j)}(x)=e^{-\mu_{j} x} \Phi(x) Q_{j}, \quad x \geq \tau,
$$

has a unique bounded matrix-valued solution on $[\tau, \infty)$ that can be obtained by iterations similar to (6.19). We note that if $j=1$, then equation (7.19) is a Volterra integral equation, and there is no need to pass to a large $\tau$ to ensure the contraction property: Indeed, the Volterra integral operator $F_{\mu_{1}}^{\tau, 1}$ has zero spectral radius on $C_{\mathrm{b}}([\tau, \infty))^{d \times d}$ for any $\tau \geq 0$ and one can start the iteration process to obtain the solution even at $\tau=0$ (cf. the proof of Theorem 3.2). In particular, this shows the uniqueness part of $(i i)$. If $j=2, \ldots, k_{0}$, then the generalized Jost solution $Y_{+}^{(j)}$ on $\mathbb{R}_{+}$is first constructed on the interval $[\tau, \infty)$ by $Y_{+}^{(j)}(x)=e^{\mu_{j} x} Z^{(j)}(x), x \geq \tau$, and then extended to $[0, \tau)$ by solving the initial value problem

$$
\left(Y_{+}^{(j)}\right)^{\prime}(x)=(A(x)+R(x)) Y_{+}^{(j)}(x), \quad x \in[0, \tau], \quad Y_{+}^{(j)}(\tau)=e^{\mu_{j} \tau} Z^{(j)}(\tau) .
$$

One verifies as in the proof of Theorem 6.5 that (7.3) and (7.4) hold. (We note the fact that for $j=2, \ldots, k_{0}$, the generalized matrix-valued Jost solutions depend on $\tau)$. This yields the existence of the generalized Jost solutions associated with the exponential splitting $Q=\sum_{j=1}^{k_{0}} Q_{j}$ of the dichotomy projection $Q$.

Inclusion (7.13) in (ii), and assertions (iii) and (iv) follow from (7.3), (7.4) and the elementary properties of Lyapunov exponents of $\mathbb{C}^{d}$-valued solutions of differential equations listed in Remark 2.7. Indeed, turning to the proof of (7.13) and (7.14), we first remark that for $j=1, \ldots, k_{0}$,

$$
\limsup _{x \rightarrow \infty} \frac{\log \left\|Y_{+}^{(j)}(x)\right\|_{\mathbb{C}^{d \times d}}}{x} \leq \max \left\{\limsup _{x \rightarrow \infty} \frac{\log \left\|Y_{+}^{(j)}(x)-\Phi(x) Q_{j}\right\|_{\mathbb{C}^{d \times d}}}{x}, \lambda_{+}\left(Q_{j}\right)\right\}
$$

does not exceed $\varkappa_{+}\left(Q_{j}\right)=\varkappa_{+}\left(P_{j}\right)$ by (7.3). Thus, the Lyapunov exponent $\lambda_{+}(y)$ of the $\mathbb{C}^{d}$-valued solution $y$ of $(1.2)$ with initial data $y(0) \in \operatorname{ran}\left(Y_{+}^{(j)}(0)\right)$ is strictly smaller than $\lambda_{+}^{\prime}\left(P_{j+1}\right)$ for $j=1, \ldots, k_{0}-1$ and is negative for $j=k_{0}$. Applying (2.19) to the perturbed equation (1.2), we obtain the inclusion $\operatorname{ran}\left(Y_{+}^{(j)}(0)\right) \subseteq$ $\operatorname{ran}\left(\sum_{k=1}^{j} P_{k}\right)$ for $j=1, \ldots, k_{0}$. To finish the proof of $(7.14)$ for $j=2, \ldots, k_{0}$, let us suppose that there is a nonzero vector $y_{0}=Y_{+}^{(j)}(0) y_{1}$, with some $y_{1} \in \mathbb{C}^{d}$, such that $y_{0} \in \operatorname{ran}\left(\sum_{k=1}^{j-1} P_{k}\right)$. By (7.4) we have $Q_{j} y_{1} \neq 0$. Then the Lyapunov 
exponent of the $\mathbb{C}^{d}$-valued solution $y$ of the initial value problem (6.27) given by $y(x)=Y_{+}^{(j)}(x) y_{1}, x \geq 0$, must satisfy $\lambda_{+}(y) \leq \varkappa_{+}\left(P_{j-1}\right)$ by Remark 2.7. This leads to a contradiction proving (7.14). Indeed, adding and subtracting $y(x)=Y_{+}^{(j)}(x) y_{1}$ in $\left\|\Phi(x) Q_{j} y_{1}\right\|_{\mathbb{C}^{d}}$, we conclude from (7.3) that

$$
\begin{aligned}
\limsup _{x \rightarrow \infty} & \frac{\log \left(\left\|\Phi(x) Q_{j} y_{1}\right\|_{\mathbb{C}^{d}}\right)}{x} \\
& \leq \max \left\{\limsup _{x \rightarrow \infty} \frac{\log \left\|Y_{+}^{(j)}(x)-\Phi(x) Q_{j}\right\|_{\mathbb{C}^{d \times d}}}{x}, \varkappa_{+}\left(P_{j-1}\right)\right\} \\
& <\varkappa_{+}^{\prime}\left(Q_{j}\right) .
\end{aligned}
$$

But this is impossible since the Lyapunov exponent of the nonzero $\mathbb{C}^{d}$-valued solution $\Phi Q_{j} y_{1}$ of (1.1) on $\mathbb{R}_{+}$with initial data $Q_{j} y_{1} \in \operatorname{ran}\left(Q_{j}\right)$ must belong to the Bohl segment $\left[\varkappa_{+}^{\prime}\left(Q_{j}\right), \varkappa_{+}\left(Q_{j}\right)\right]$ (see again Remark 2.7).

Finally, to verify assertion $(i v)$ we add and subtract $\Phi(x) Q_{j}$ in $Y_{+}^{(j)}(x)-\widetilde{Y}_{+}^{(j)}(x)$ and use (7.3) to conclude that $\lambda_{+}(y)<\lambda_{+}^{\prime}\left(P_{j}\right)$ for every $\mathbb{C}^{d}$-valued solution $y$ of (1.2) with $y(0) \in \operatorname{ran}\left(Y_{+}^{(j)}(0)-\widetilde{Y}_{+}^{(j)}(0)\right)$. This proves inclusion (7.16).

Remark 7.4. In addition to assertions $(i i)-(i v)$ in Theorem 7.3 , the generalized matrix-valued Jost solutions $Y_{ \pm}^{(j)}$, constructed in the existence part $(i)$ of Theorem 7.3 by means of the Fredholm-type integral equations (7.19) and their analogs for $\mathbb{R}_{-}$, have the following property: If $R_{n}, n \in \mathbb{N}$, is the sequence of truncated perturbations as in (4.14), and $Y_{ \pm, n}^{(j)}$ denote the generalized matrix-valued Jost solutions associated with the perturbation $R_{n}$ (see the proof of Theorem 4.1), then

$$
\lim _{n \rightarrow \infty} Y_{ \pm, n}^{(j)}(0)=Y_{ \pm}^{(j)}(0)
$$

To see this one follows the course of the proof of Theorem 6.5. First one establishes continuity of the map $R \mapsto Z^{(j)}$ from $L^{1}\left(\mathbb{R}_{+} ; e^{\beta x} d x\right)$ to $C_{\mathrm{b}}([\tau, \infty))^{d \times d}$, defined in a neighborhood $\mathcal{U}(R)$ of $R$ in the space $L^{1}\left(\mathbb{R}_{+} ; e^{\beta x} d x\right)$. Subsequently, one shows that the convergence of the sequence $\left\{R_{n}\right\}_{n \in \mathbb{N}}$ from this neighborhood to $R$ as $n \rightarrow \infty$ in the $L^{1}\left(\mathbb{R}_{+} ; e^{\beta x} d x\right)$-topology yields $(7.24)$.

Next, given any set of generalized matrix-valued Jost solutions, we introduce the following Evans determinant (the terminology is related to the Evans function, which is further discussed in Section 9).

Definition 7.5. For a given set of generalized matrix-valued Jost solutions $Y_{+}^{(j)}$, $j=1, \ldots, k_{0}$, on $\mathbb{R}_{+}$and $Y_{-}^{(j)}, j=k_{0}+1 \ldots, d^{\prime}$, on $\mathbb{R}_{-}$, the Evans determinant, $D$, is defined by

$$
D=\operatorname{det}_{\mathbb{C}^{d}}\left(\mathcal{Y}_{+}+\mathcal{Y}_{-}\right), \text {where } \mathcal{Y}_{+}=\sum_{j=1}^{k_{0}} Y_{+}^{(j)}(0), \quad \mathcal{Y}_{-}=\sum_{j=k_{0}+1}^{d^{\prime}} Y_{-}^{(j)}(0) .
$$

The following important and purely algebraic result shows that although the generalized matrix-valued Jost solutions are not unique, the Evans determinant $D$ is uniquely determined by equations (1.1) and (1.2). 
Lemma 7.6. Assume Hypothesis 7.1. Then the determinant $D$ in (7.25) is independent of the choice of the generalized matrix-valued Jost solutions $Y_{+}^{(j)}, j=$ $1, \ldots, k_{0}$, and $Y_{-}^{(j)}, j=k_{0}+1 \ldots, d^{\prime}$.

Proof. Using the notation (7.7) and (7.9), let $P_{+}$and $P_{-}$be the dichotomy projections and $\left\{P_{j}\right\}_{j=1}^{k_{0}}$ and $\left\{P_{j}\right\}_{j=k_{0}+1}^{d^{\prime}}$ be the exponential splittings for $(1.2)$ on $\mathbb{R}_{+}$and $\mathbb{R}_{-}$, having the same Bohl segments as the splitting $\left\{Q_{j}\right\}_{j=1}^{d^{\prime}}$ for (1.1). Without loss of generality, we will assume that the subspaces $\operatorname{ran}\left(P_{j}\right), j=1, \ldots, d^{\prime}$, satisfy $\operatorname{ran}\left(P_{1}\right) \dot{+} \operatorname{ran}\left(P_{2}\right) \dot{+} \cdots \dot{+} \operatorname{ran}\left(P_{d^{\prime}}\right)=\mathbb{C}^{d}$ (otherwise the determinant $D$ is equal to zero for any choice of the system of the generalized Jost solutions). We note that $\operatorname{ran}\left(\mathcal{Y}_{ \pm}\right) \subseteq \operatorname{ran}\left(P_{ \pm}\right)$by assertions $(i i)$ and $(i i i)$ in Theorem 7.3, and $\mathcal{Y}_{+}=\mathcal{Y}_{+} Q$ and $\mathcal{Y}_{-}=\mathcal{Y}_{-}\left(I_{d}-Q\right)$ by (7.4) and (7.6). Thus, treating the matrices $\mathcal{Y}_{ \pm}$in $(7.25)$ as operators $\mathcal{Y}_{+}: \operatorname{ran}(Q) \rightarrow \operatorname{ran}\left(P_{+}\right)$and $\mathcal{Y}_{-}: \operatorname{ran}\left(I_{d}-Q\right) \rightarrow \operatorname{ran}\left(P_{-}\right)$, we observe that the block-operator $\mathcal{Y}=\mathcal{Y}_{+}+\mathcal{Y}_{-}$is diagonal in the following direct sum decomposition:

$$
\mathcal{Y}=\left(\begin{array}{cc}
\mathcal{Y}_{+} & 0 \\
0 & \mathcal{Y}_{-}
\end{array}\right): \mathbb{C}^{d}=\operatorname{ran}(Q) \dot{+} \operatorname{ran}\left(I_{d}-Q\right) \rightarrow \mathbb{C}^{d}=\operatorname{ran}\left(P_{+}\right) \dot{+} \operatorname{ran}\left(P_{-}\right)
$$

Assertions (ii) and (iii) of Theorem 7.3 then also yield that the matrix of the operator

$$
\begin{aligned}
& \mathcal{Y}_{+}: \operatorname{ran}(Q)=\operatorname{ran}\left(Q_{1}\right) \dot{+} \cdots \dot{+} \operatorname{ran}\left(Q_{k_{0}}\right) \\
& \quad \rightarrow \operatorname{ran}\left(P_{+}\right)=\operatorname{ran}\left(P_{1}\right) \dot{+} \cdots \dot{+} \operatorname{ran}\left(P_{k_{0}}\right)
\end{aligned}
$$

is upper-triangular, while the matrix of the operator

$$
\begin{aligned}
\mathcal{Y}_{-}: \operatorname{ran}\left(I_{d}-Q\right)=\operatorname{ran}\left(Q_{k_{0}+1}\right) \dot{+} \cdots \dot{+} \operatorname{ran}\left(Q_{d^{\prime}}\right) \\
\rightarrow \operatorname{ran}\left(P_{+}\right)=\operatorname{ran}\left(P_{k_{0}+1}\right) \dot{+} \cdots \dot{+} \operatorname{ran}\left(P_{d^{\prime}}\right)
\end{aligned}
$$

is lower-triangular. Moreover, from assertion $(i v)$ in Theorem 7.3 it also follows that the diagonal blocks of the operators $\mathcal{Y}_{ \pm}$with respect to the decompositions (7.27) and (7.28) are independent of the choice of the generalized Jost solutions, completing the proof.

Remark 7.7. In fact, the proof of Lemma 7.6 only uses properties (7.4) and (7.6), and properties (7.13)-(7.15) of the matrix-valued solutions of (1.2).

At this point we are ready to prove the principal result of this paper.

Theorem 7.8. Assume Hypothesis 7.1 and the condition

$$
\|R\|_{\mathbb{C}^{d \times d}} \in L^{1}\left(\mathbb{R} ; e^{\beta|x|} d x\right)
$$

for some

$$
\beta>\max \left\{\max _{1 \leq j \leq k_{0}}\left\{\lambda_{+}\left(Q_{j}\right)-\varkappa_{+}^{\prime}\left(Q_{j}\right)\right\}, \max _{k_{0}+1 \leq j \leq d^{\prime}}\left\{\varkappa_{-}\left(Q_{j}\right)-\lambda_{-}^{\prime}\left(Q_{j}\right)\right\}\right\} .
$$

Let $Y_{+}^{(j)}, j=1,2, \ldots, k_{0}$, and $Y_{-}^{(j)}, j=k_{0}+1,2, \ldots, d^{\prime}$, be any system of matrixvalued generalized Jost solutions. Let $K$ be the integral operator on $L^{2}(\mathbb{R})^{d}$ whose integral kernel is given by (2.25). Then the 2-modified perturbation determinant $\operatorname{det}_{2}(I+K)$ admits the representation

$$
\operatorname{det}_{2}(I+K)=e^{\Theta} \operatorname{det}_{\mathbb{C}^{d}}\left(\mathcal{Y}_{+}+\mathcal{Y}_{-}\right),
$$


where $\Theta$ is defined in (4.3), $\mathcal{Y}_{ \pm}$are defined in (7.25), and $D=\operatorname{det}_{\mathbb{C}^{d}}\left(\mathcal{Y}_{+}+\mathcal{Y}_{-}\right)$is the Evans determinant (cf. (7.25)).

Proof. Let $Y_{+, F}^{(j)}, j=1, \ldots, k_{0}$, and $Y_{-, F}^{(j)}, j=k_{0}+1, \ldots, d^{\prime}$, be the generalized matrix-valued Jost solutions on $\mathbb{R}_{+}$, respectively, on $\mathbb{R}_{-}$, constructed in the existence part $(i)$, (ii) of the proof of Theorem 7.3 for a given $\tau \geq 0$ sufficiently large. Here, the subscript $F$ is added to remind the reader that these solutions are obtained from solutions of the Fredholm-type integral equations (7.19) and their analogs for $\mathbb{R}_{-}$. In general we note that the solutions $Y_{ \pm, F}^{(j)}$ depend on $\tau$, and they are not the solutions $Y_{ \pm}^{(j)}$ given a priori in the formulation of Theorem 7.8. Introduce the truncated perturbations $R_{n}, n \in \mathbb{N}$, by formula (4.14), and denote by $Y_{+, n, F}^{(j)}, j=1, \ldots, k_{0}$, and $Y_{-, n, F}^{(j)}, j=k_{0}+1, \ldots, d^{\prime}$, the corresponding generalized matrix-valued Jost solutions of the truncated perturbed equation $y^{\prime}(x)=\left(A(x)+R_{n}(x)\right) y(x)$, obtained by solving the Fredholm-type integral equations (7.19) with $R$ replaced by $R_{n}$ (see the proof of Theorem 7.3(i)). Since $R_{n}$ is compactly supported, there exist unique matrix-valued Jost solutions in the sense of Definition 3.1 of the truncated perturbed equation on $\mathbb{R}_{+}$and on $\mathbb{R}_{-}$; these will be denoted by $Y_{+, n}$ and $Y_{-, n}$. Using the solutions $Y_{ \pm, n}$ and the projections $Q_{j}$, define matrix-valued solutions of the truncated perturbed equation by

$$
\begin{aligned}
& Y_{+, n, V}^{(j)}(x)=Y_{+, n}(x) Q_{j}, j=1, \ldots, k_{0}, x \geq 0, \\
& Y_{-, n, V}^{(j)}(x)=Y_{-, n}(x) Q_{j}, j=k_{0}+1, \ldots, d^{\prime}, x \leq 0 .
\end{aligned}
$$

Here, the subscript $V$ is added to remind the reader that the solutions $Y_{ \pm, n}$ are obtained from the Volterra integral equations (4.26). Since the support of $R_{n}$ is compact, equations (4.26) imply that the solutions $Y_{+, n, V}^{(j)}$ constitute a system of generalized matrix-valued Jost solutions in the sense of Definition 7.2. In addition to $(7.25)$, introduce the notations

$$
\begin{aligned}
\mathcal{Y}_{+, F} & =\sum_{j=1}^{k_{0}} Y_{+, F}^{(j)}(0), & \mathcal{Y}_{-, F} & =\sum_{j=k_{0}+1}^{d^{\prime}} Y_{-, F}^{(j)}(0), \\
\mathcal{Y}_{+, n, F} & =\sum_{j=1}^{k_{0}} Y_{+, n, F}^{(j)}(0), & \mathcal{Y}_{-, n, F} & =\sum_{j=k_{0}+1}^{d^{\prime}} Y_{-, n, F}^{(j)}(0), \\
\mathcal{Y}_{+, n, V} & =\sum_{j=1}^{k_{0}} Y_{+, n, V}^{(j)}(0), & \mathcal{Y}_{-, n, V} & =\sum_{j=k_{0}+1}^{d^{\prime}} Y_{-, n, V}^{(j)}(0) .
\end{aligned}
$$

Using (4.27) and the definition of $Y_{ \pm, n, V}^{(j)}$ in (7.32) we obtain

$$
\mathcal{Y}_{+, n, V}=\sum_{j=1}^{k_{0}} Y_{+, n}(0) Q_{j}=Y_{+, n}(0) Q=Y_{+, n}(0)
$$

and similarly,

$$
\mathcal{Y}_{-, n, V}=Y_{-, n}(0)
$$

Using Remark 4.3 this results in

$$
\operatorname{det}_{2}(I+K)=e^{\Theta} \lim _{n \rightarrow \infty} \operatorname{det}_{\mathbb{C}^{d}}\left(\mathcal{Y}_{+, n, V}+\mathcal{Y}_{-, n, V}\right) .
$$


Since both $Y_{ \pm, n, V}^{(j)}$ and $Y_{ \pm, n, F}^{(j)}$ constitute systems of generalized matrix-valued Jost solutions, Lemma 7.6 yields

$$
\operatorname{det}_{\mathbb{C}^{d}}\left(\mathcal{Y}_{+, n, V}+\mathcal{Y}_{-, n, V}\right)=\operatorname{det}_{\mathbb{C}^{d}}\left(\mathcal{Y}_{+, n, F}+\mathcal{Y}_{-, n, F}\right), \quad n \in \mathbb{N} .
$$

By the continuity argument discussed in Remark 7.4 (cf. (7.24)), we get

$$
\lim _{n \rightarrow \infty} \operatorname{det}_{\mathbb{C}^{d}}\left(\mathcal{Y}_{+, n, F}+\mathcal{Y}_{-, n, F}\right)=\operatorname{det}_{\mathbb{C}^{d}}\left(\mathcal{Y}_{+, F}+\mathcal{Y}_{-, F}\right)
$$

and next, again by Lemma 7.6,

$$
\operatorname{det}_{\mathbb{C}^{d}}\left(\mathcal{Y}_{+, F}+\mathcal{Y}_{-, F}\right)=\operatorname{det}_{\mathbb{C}^{d}}\left(\mathcal{Y}_{+}+\mathcal{Y}_{-}\right) .
$$

Combining (7.38)-(7.41) yields (7.31), completing the proof.

Next, we will give an extension of Lemma 7.6 and Theorem 7.8 for the case where the matrix-valued solutions $\Phi Q_{j}, j=1, \ldots, d^{\prime}$, of (1.1) in Definition 7.2 are replaced by arbitrary solutions of (1.1) with certain asymptotic properties. We assume Hypothesis 7.1 and fix the splitting $\left\{P_{j}\right\}_{j=1}^{d^{\prime}}$ for (1.2) described in (7.7) and (7.9). Let $N_{j}, j=1, \ldots, d^{\prime}$, be any matrices in $\mathbb{C}^{d \times d}$ satisfying the following conditions:

$$
\begin{gathered}
\text { (i) } N_{j}=N_{j} Q_{j}=Q_{j} N_{j}, \quad j=1, \ldots, d^{\prime} . \\
\text { (ii) } \operatorname{det}_{\mathbb{C}^{d}}(N) \neq 0, \quad \text { where } \quad N=\sum_{j=1}^{d^{\prime}} N_{j} .
\end{gathered}
$$

Let $\Phi_{j}$ for $j=1, \ldots, k_{0}$, respectively, $j=k_{0}+1, \ldots, d^{\prime}$, denote the matrix-valued solutions of the unperturbed equation (1.1) on $\mathbb{R}_{+}$, respectively, $\mathbb{R}_{-}$, satisfying the initial conditions $\Phi_{j}(0)=N_{j}, j=1, \ldots, d^{\prime}$. By $(i)$ in (7.42), the Lyapunov exponents of the $\mathbb{C}^{d}$-valued solutions of (1.1) given by the columns of $\Phi_{j}$ belong to the Bohl segments corresponding to the projections $Q_{j}, j=1, \ldots, d^{\prime}$. Next, assume that $\tilde{Y}_{+}^{(j)}, j=1,2, \ldots, k_{0}$, on $\mathbb{R}_{+}$, and $\widetilde{Y}_{-}^{(j)}, j=k_{0}+1,2, \ldots, d^{\prime}$, on $\mathbb{R}_{-}$, are any given $d \times d$ matrix-valued solutions of the differential equation $Y^{\prime}(x)=$ $(A(x)+R(x)) Y(x), x \in \mathbb{R}_{ \pm}$, satisfying the following properties (cf. Definition 7.2):

$$
\begin{aligned}
& \limsup _{x \rightarrow \infty} \frac{\log \left\|\widetilde{Y}_{+}^{(j)}(x)-\Phi_{j}(x)\right\|_{\mathbb{C}^{d \times d}}}{x}<\varkappa_{+}^{\prime}\left(Q_{j}\right), \\
& \widetilde{Y}_{+}^{(j)}(0)=\widetilde{Y}_{+}^{(j)}(0) Q_{j}, \quad j=1,2, \ldots, k_{0},
\end{aligned}
$$

and

$$
\begin{aligned}
& \liminf _{x \rightarrow-\infty} \frac{\log \left\|\tilde{Y}_{-}^{(j)}(x)-\Phi_{j}(x)\right\|_{\mathbb{C}^{d \times d}}}{x}>\varkappa_{-}\left(Q_{j}\right), \\
& \tilde{Y}_{-}^{(j)}(0)=\tilde{Y}_{-}^{(j)}(0) Q_{j}, \quad j=k_{0}+1, \ldots, d^{\prime} .
\end{aligned}
$$

Define the following matrix $\widetilde{N}$ (cf. Definition 7.5):

$$
\tilde{N}=\tilde{\mathcal{Y}}_{+}+\widetilde{\mathcal{Y}}_{-} \text {, where } \tilde{\mathcal{Y}}_{+}=\sum_{j=1}^{k_{0}} \widetilde{Y}_{+}^{(j)}(0), \quad \widetilde{\mathcal{Y}}_{-}=\sum_{j=k_{0}+1}^{d^{\prime}} \tilde{Y}_{-}^{(j)}(0) .
$$

We remark that if $N_{j}=Q_{j}$ in $(7.42)$, then $\operatorname{det}_{\mathbb{C}^{d}}(N)=1$ and $\widetilde{Y}_{ \pm}^{(j)}$ are generalized matrix-valued Jost solutions $Y_{ \pm}^{(j)}$ in the sense of Definition 7.2 , and $\widetilde{N}=\mathcal{Y}_{+}+\mathcal{Y}_{-}$, in the notations of (7.25). 
Lemma 7.9. Assume Hypothesis 7.1. In addition suppose that $N_{j}$ satisfy (7.42), that $\Phi_{j}$ satisfy $\Phi_{j}(0)=N_{j}$, and that $\tilde{Y}_{ \pm}^{(j)}$ satisfy conditions $(7.43)-(7.46)$ for $j=$ $1, \ldots, d^{\prime}$. Finally, assume that $\widetilde{N}$ is defined as in (7.47). Then the ratio of the determinants $\operatorname{det}_{\mathbb{C}^{d}}(\widetilde{N})$ and $\operatorname{det}_{\mathbb{C}^{d}}(N)$ is independent of the choice of the solutions $\Phi_{j}$ with the initial data satisfying (7.42) and the choice of the solutions $\widetilde{Y}_{ \pm}^{(j)}, j=$ $1, \ldots, d^{\prime}$. Thus, one obtains for the Evans determinant $D$ in Definition 7.5 ,

$$
D=\frac{\operatorname{det}_{\mathbb{C}^{d}}(\widetilde{N})}{\operatorname{det}_{\mathbb{C}^{d}}(N)}
$$

Proof. Using (ii) in (7.42), we denote $Y_{ \pm}^{(j)}(x)=\widetilde{Y}_{ \pm}^{(j)}(x) N^{-1}, x \in \mathbb{R}_{ \pm}$, for $j=$ $1, \ldots, d^{\prime}$. We claim that $Y_{ \pm}^{(j)}$ are generalized matrix-valued Jost solutions in the sense of Definition 7.2. Indeed, since $Q_{j}=N_{j} N^{-1}$ due to $N_{j}=Q_{j} N$, the equalities

$$
Y_{+}^{(j)}(x)-\Phi(x) Q_{j}=\widetilde{Y}_{+}^{(j)}(x) N^{-1}-\Phi(x) N_{j} N^{-1}=\left(\widetilde{Y}_{+}^{(j)}(x)-\Phi_{j}(x)\right) N^{-1}
$$

show that condition (7.3) follows from (7.43). Also, since $N^{-1}$ and $Q_{j}$ commute, condition (7.44) implies (7.4), proving the claim. By Lemma 7.6, the Evans determinant $D$ constructed using the solutions $Y_{ \pm}^{(j)}$ as described in Definition 7.5 , is independent of the choice of $N_{j}$ and $\widetilde{Y}_{ \pm}^{(j)}$, and hence equals

$$
\begin{aligned}
D & =\operatorname{det}_{\mathbb{C}^{d}}\left(\mathcal{Y}_{+}+\mathcal{Y}_{-}\right)=\operatorname{det}_{\mathbb{C}^{d}}\left(\sum_{j=1}^{k_{0}} \widetilde{Y}_{+}^{(j)}(0) N^{-1}+\sum_{j=k_{0}+1}^{d^{\prime}} \widetilde{Y}_{-}^{(j)}(0) N^{-1}\right) \\
& =\operatorname{det}_{\mathbb{C}^{d}}\left(\widetilde{N} N^{-1}\right),
\end{aligned}
$$

proving (7.48).

Corollary 7.10. Under the assumptions imposed in Theorem 7.8 and Lemma 7.9, the 2-modified perturbation determinant $\operatorname{det}_{2}(I+K)$ admits the representation

$$
\operatorname{det}_{2}(I+K)=e^{\Theta} \frac{\operatorname{det}_{\mathbb{C}^{d}}(\widetilde{N})}{\operatorname{det}_{\mathbb{C}^{d}}(N)}
$$

where $\Theta$ is defined in (4.3), and $N$ and $\widetilde{N}$ are defined in (7.42) and (7.47).

Remark 7.11. Theorem 7.3 can be viewed as a further development of the celebrated Levinson theorem (see, e.g., [13, Chap.1] and the bibliography cited therein). The Levinson theorem for the asymptotically diagonal perturbed equation (1.2) on $\mathbb{R}_{+}$deals with the situation when $A(x)=\operatorname{diag}\left\{\lambda_{j}(x)\right\}_{j=1}^{d}, x \in \mathbb{R}_{+}$, and $\|R\|_{\mathbb{C}^{d \times d}} \in$ $L^{1}\left(\mathbb{R}_{+}\right)$and asserts the existence of $\mathbb{C}^{d}$-valued solutions of (1.2) satisfying the asymptotic relation

$$
y_{j}(x) \underset{x \rightarrow \infty}{=}\left(\mathbf{e}_{j}+o(1)\right) \exp \int_{0}^{x} \lambda_{j}(s) d s, \quad j=1, \ldots, d,
$$

where $\mathbf{e}_{j}, j=1, \ldots, d$, are the vectors of the standard basis in $\mathbb{C}^{d}$. The underlying assumption in the traditional Levinson theorem (i.e., for (7.52) to be valid) is that the following alternative holds: For each pair of integers $j, k \in\{1, \ldots, d\}, j \neq k$, either

$$
\liminf _{x \rightarrow \infty} I_{j, k}(0, x)=-\infty \text { and } \sup _{x \geq x^{\prime} \geq 0} I_{j, k}\left(x^{\prime}, x\right)<\infty
$$


or

$$
\inf _{x \geq x^{\prime} \geq 0} I_{j, k}\left(x, x^{\prime}\right)>-\infty
$$

where we denote $I_{j, k}\left(x^{\prime}, x\right)=\int_{x^{\prime}}^{x} d s \operatorname{Re}\left(\lambda_{j}(s)-\lambda_{k}(s)\right), 0 \leq x^{\prime} \leq x$. To compare our results and the traditional Levinson theorem, we first note that, unlike the assumptions in the Levinson theorem, we do not assume that $A$ is diagonal. In the class of diagonal unperturbed equations (1.1) our assumptions on the unperturbed diagonal system are more special (since we are interested only in bounded solutions on $\mathbb{R}_{+}$, and assume the exponential dichotomy on $\mathbb{R}$ ), but in turn we derive more conclusions in addition to the asymptotic relation (7.52), see assertions (ii)-(iv) in Theorem 7.3. If, in addition, we assume that the diagonal unperturbed system has $d$ disjoint Bohl segments, then the above mentioned alternative holds; thus our assumptions are stronger than that in the Levinson theorem in this particular case. However, generally, our hypotheses are more flexible since we can group the diagonal elements of $A$ related to the same Bohl segment, thus avoiding conditions on all pairs $j, k$ as in the traditional Levinson theorem. Our assumptions (7.11) on the perturbation are stronger than those in the Levinson theorem, but in turn (cf. (7.52)), we conclude that the columns of the generalized matrix-valued Jost solutions approximate the reference solutions up to terms $o\left(e^{-\delta x}\right)$ as $x \rightarrow \infty$ for a positive $\delta$ (cf. (7.3) and (6.25)). Finally, the generalized matrix-valued Jost solutions yield formula (7.31) for the perturbation determinant, a feature that is not discussed in the context of the traditional Levinson theorem.

\section{Autonomous Perturbed Equations}

In this section we treat the case of an autonomous unperturbed equation (1.1), that is, we consider the differential equations

$$
\begin{aligned}
& y^{\prime}(x)=A y(x), \quad x \in \mathbb{R}, \\
& y^{\prime}(x)=(A+R(x)) y(x), \quad x \in \mathbb{R},
\end{aligned}
$$

on $\mathbb{R}$, where $A \in \mathbb{C}^{d \times d}$ is a constant matrix and $R \in L_{\text {loc }}^{1}(\mathbb{R})^{d \times d}$ (cf. Examples 2.3, 2.4 ), and 2.6). Our objective is to show that the conclusions of Theorems 7.3 and 7.8 hold for (8.1) and (8.2) under much weaker assumptions on the perturbation $R$ than the exponential decay imposed in (7.11) and (7.12). Specifically, we will merely assume that $\|R\|_{\mathbb{C}^{d \times d}} \in L^{1}(\mathbb{R})$ when the eigenvalues of $A$ are semi-simple, or $\|R\|_{\mathbb{C}^{d \times d}}$ decays polynomially when $A$ has non-diagonal Jordan blocks. Under these weaker assumptions, the asymptotic behavior of the generalized matrix-valued Jost solutions will no longer be measured in terms of exponential weight factors as indicated in Definition 7.2. Instead, we will define the generalized matrix-valued Jost solutions of (8.2) by means of solutions of a certain mixed system of Volterraand Fredholm-type integral equations in the spirit of Definition 5.2.

Hypothesis 8.1. Assume that $d \geq 2, A \in \mathbb{C}^{d \times d}$, and $\sigma(A) \cap i \mathbb{R}=\emptyset$. Let $Q_{j}$ denote the spectral projection of $A$ corresponding to the spectral subset of the eigenvalues of $A$ having equal real parts denoted by $\varkappa_{j}, j=1, \ldots, d^{\prime}$, where $2 \leq d^{\prime} \leq d$. In addition, assume that for some $k_{0}, 1 \leq k_{0} \leq d^{\prime}-1$, the inequalities $\varkappa_{1}<\cdots<\varkappa_{k_{0}}<$ $0<\varkappa_{k_{0}+1}<\cdots<\varkappa_{d^{\prime}}$ hold, so that $Q=\sum_{j=1}^{k_{0}} Q_{j}$ is the dichotomy projection for (8.1) on $\mathbb{R}$ and on $\mathbb{R}_{ \pm}$. 
Passing to an appropriate coordinate system, we may assume that the matrix $A$ is in Jordan normal form, and thus for each $j=1, \ldots, d^{\prime}$ the operator $\left.A\right|_{\operatorname{ran}\left(Q_{j}\right)}$ is represented by a direct sum of diagonal matrices $\nu I$ and/or matrices $\nu I+J$, where $\nu \in \sigma(A), \operatorname{Re}(\nu)=\varkappa_{j}$, and $J$ is the matrix of an appropriate size with 1 's above the main diagonal and all other entries equal to zero. We introduce $m_{j} \in \mathbb{N}$ such that $m_{j}+1$ is equal to the maximal size of the non-diagonal Jordan blocks of $\left.A\right|_{\operatorname{ran}\left(Q_{j}\right)}$, noting that $m_{j} \geq 1$ if such blocks exists, and setting $m_{j}=0$ if all Jordan blocks are diagonal (i.e., if all eigenvalues $\nu$ of $A$ with $\operatorname{Re}(\nu)=\varkappa_{j}$ are semi-simple). Clearly, the matrix exponent $e^{\left.A\right|_{\operatorname{ran}\left(Q_{j}\right)}}$ can be computed explicitly using the Jordan blocks of $\left.A\right|_{\operatorname{ran}\left(Q_{j}\right)}$ (see, e.g., [14, Example I.2.5]), so that with this notation one has the estimate

$$
\left\|e^{\left.x A\right|_{\operatorname{ran}\left(Q_{j}\right)}}\right\| \leq c e^{\varkappa_{j} x}(1+|x|)^{m_{j}}, \quad j=1, \ldots, d^{\prime}, \quad x \in \mathbb{R},
$$

for some constant $c>0$. Finally, we introduce $m=\max _{1 \leq j \leq d^{\prime}}\left\{m_{j}\right\}$.

Next, we fix $\tau>0$, and consider the following "mixed" system of Volterraand Fredholm-type integral equations (cf. (5.6) with the weight function $f(x)=$ $(1+|x|)^{m_{j}}, x \in \mathbb{R}$, and (7.19) with $\left.\mu_{j}=\varkappa_{j}\right)$ :

$$
\begin{aligned}
& Z_{+}^{(j)}(x)-(1+|x|)^{-m_{j}} e^{x\left(A-\varkappa_{j}\right)} Q_{j} \\
& =-\int_{x}^{\infty} d x^{\prime} e^{\left(x-x^{\prime}\right)\left(A-\varkappa_{j}\right)}(1+|x|)^{-m_{j}}\left(\sum_{k=j}^{d^{\prime}} Q_{k}\right)\left(1+\left|x^{\prime}\right|\right)^{m_{j}} R\left(x^{\prime}\right) Z_{+}^{(j)}\left(x^{\prime}\right) \\
& \quad+\int_{\tau}^{x} d x^{\prime} e^{\left(x-x^{\prime}\right)\left(A-\varkappa_{j}\right)}(1+|x|)^{-m_{j}}\left(\sum_{k=1}^{j-1} Q_{k}\right)\left(1+\left|x^{\prime}\right|\right)^{m_{j}} R\left(x^{\prime}\right) Z_{+}^{(j)}\left(x^{\prime}\right), \\
& Z_{-}^{(j)}(x)-(1+|x|)^{-m_{j}} e^{x\left(A-\varkappa_{j}\right)} Q_{j} \\
& =\int_{-\infty}^{x} d x^{\prime} e^{\left(x-x^{\prime}\right)\left(A-\varkappa_{j}\right)}(1+|x|)^{-m_{j}}\left(\sum_{k=1}^{j} Q_{k}\right)\left(1+\left|x^{\prime}\right|\right)^{m_{j}} R\left(x^{\prime}\right) Z_{-}^{(j)}\left(x^{\prime}\right) \\
& \quad-\int_{x}^{-\tau} d x^{\prime} e^{\left(x-x^{\prime}\right)\left(A-\varkappa_{j}\right)}(1+|x|)^{-m_{j}}\left(\sum_{k=j+1}^{d^{\prime}} Q_{k}\right)\left(1+\left|x^{\prime}\right|\right)^{m_{j}} R\left(x^{\prime}\right) Z_{-}^{(j)}\left(x^{\prime}\right), \\
& j=k_{0}+1, \ldots, d^{\prime}, \quad x \leq-\tau .
\end{aligned}
$$

Here, we set $\sum_{k=1}^{j-1} Q_{k}=0$ when $j=1$ in (8.4) and $\sum_{k=j+1}^{d^{\prime}} Q_{k}=0$ when $j=d^{\prime}$ in (8.5); thus, the first and the last equations in (8.4) and (8.5) are of Volterra-type, and the remaining equations are of Fredholm-type.

Definition 8.2. Assume Hypothesis 8.1 and $R \in L_{\text {loc }}^{1}(\mathbb{R})^{d \times d}$. Then $d \times d$ matrixvalued solutions $Y_{+}^{(j)}, j=1, \ldots, k_{0}$, on $\mathbb{R}_{+}$and $Y_{-}^{(j)}, j=k_{0}+1, \ldots, d^{\prime}$, on $\mathbb{R}_{-}$of the differential equation

$$
Y^{\prime}(x)=(A+R(x)) Y(x), \quad x \in \mathbb{R}_{ \pm},
$$

are called generalized matrix-valued Jost solutions associated with the exponential splitting $\left\{Q_{j}\right\}_{j=1}^{d^{\prime}}$ if

$$
Y_{+}^{(j)}(x)=(1+|x|)^{m_{j}} e^{\varkappa_{j} x} Z_{+}^{(j)}(x), \quad j=1, \ldots, k_{0}, \quad x \geq \tau
$$




$$
Y_{-}^{(j)}(x)=(1+|x|)^{m_{j}} e^{\varkappa_{j} x} Z_{-}^{(j)}(x), \quad j=k_{0}+1, \ldots, d^{\prime}, \quad x \leq-\tau,
$$

where $Z_{+}^{(j)}, j=1, \ldots, k_{0}$, are bounded solutions of equation $(8.4)$ on $[\tau, \infty)$ and $Z_{-}^{(j)}, j=k_{0}+1, \ldots, d^{\prime}$, are bounded solutions of equation $(8.5)$ on $(-\infty, \tau]$. The solutions $Y_{+}^{(j)}$ and $Y_{-}^{(j)}$ are extended to $[0, \tau)$ and to $(-\tau, 0]$, respectively, by solving the initial value problem for equation (8.2) with initial data $Y_{ \pm}^{(j)}( \pm \tau)=(1+$ $\tau)^{m_{j}} e^{ \pm \varkappa_{j} \tau} Z_{ \pm}^{(j)}( \pm \tau)$ for the corresponding values of $j=1, \ldots, d^{\prime}$.

We emphasize that the generalized matrix-valued Jost solutions in the sense of Definition 8.2 are not unique (unless $d^{\prime}=2$, see Definition 5.2) and depend on the choice of $\tau$ in (8.4) and (8.5). We will continue to use Definition 7.5 of the Evans determinant, but the generalized matrix-valued Jost solutions in this definition will be understood in the sense of Definition 8.2. We recall the exponential splitting $\left\{P_{j}\right\}_{j=1}^{d^{\prime}}$ in (7.7) and (7.9), for which the Bohl and Lyapunov exponents for the perturbed equation (8.2) are also equal to $\varkappa_{j}, j=1, \ldots, d^{\prime}$.

Theorem 8.3. Assume Hypothesis 8.1 and the condition

$$
\|R\|_{\mathbb{C}^{d \times d}} \in L^{1}\left(\mathbb{R} ;(1+|x|)^{2 m} d x\right),
$$

where $m+1, m \geq 1$, is the maximal size of the non-diagonal Jordan blocks of $A$ (if such blocks exist), or $m=0$ if all Jordan blocks of $A$ are diagonal. Then the following conclusions hold:

Assertions (i)-(iii) of Theorem 7.3 hold, where the generalized matrix-valued Jost solutions are understood in the sense of Definition 8.2.

In addition:

(iv) The generalized matrix-valued Jost solutions satisfy

$$
\begin{aligned}
& e^{-\varkappa_{j} x}\left\|Y_{+}^{(j)}(x)-e^{x A} Q_{j}\right\|_{\mathbb{C}^{d \times d}} \underset{x \rightarrow \infty}{=} o(1), \quad j=1, \ldots, k_{0}, \\
& e^{-\varkappa_{j} x}\left\|Y_{-}^{(j)}(x)-e^{x A} Q_{j}\right\|_{\mathbb{C}^{d \times d}} \underset{x \rightarrow-\infty}{=} o(1), \quad j=k_{0}+1, \ldots, d^{\prime} .
\end{aligned}
$$

$(v)$ The 2-modified perturbation determinant $\operatorname{det}_{2}(I+K)$ admits the representation

where

$$
\operatorname{det}_{2}(I+K)=e^{\Theta} \operatorname{det}_{\mathbb{C}^{d}}\left(\mathcal{Y}_{+}+\mathcal{Y}_{-}\right)
$$

$$
\Theta=\int_{0}^{\infty} d x \operatorname{tr}_{\mathbb{C}^{d}}[Q R(x)]-\int_{-\infty}^{0} d x \operatorname{tr}_{\mathbb{C}^{d}}\left[\left(I_{d}-Q\right) R(x)\right],
$$

$\mathcal{Y}_{ \pm}$are defined as in $(7.25)$, and $D=\operatorname{det}_{\mathbb{C}^{d}}\left(\mathcal{Y}_{+}+\mathcal{Y}_{-}\right)$is the Evans determinant $(c f .(7.25))$.

Proof. We will sketch the proof for the case of $\mathbb{R}_{+}$referring for details to the proofs of Theorem 7.3, Lemma 7.6, and Theorem 7.8. The proof for the case of $\mathbb{R}_{-}$is similar. We fix $j \in\left\{1, \ldots, k_{0}\right\}$. Starting the proof of assertion $(i)$, let $F_{\varkappa_{j}}^{\tau, j}$ denote the integral operator on $C_{\mathrm{b}}([\tau, \infty))^{d \times d}$ defined by the right-hand side of (8.4) (cf. $(7.18))$.

We claim that under assumption (8.9) the following assertions hold:

(a) $F_{\varkappa_{j}}^{\tau, j}$ is a contraction on $C_{\mathrm{b}}([\tau, \infty))^{d \times d}$ for $\tau$ sufficiently large.

(b) There exists a function $g \in C_{\mathrm{b}}([\tau, \infty))$ so that $\lim _{x \rightarrow \infty} g(x)=0$ and

$$
\left\|\left(F_{\varkappa_{j}}^{\tau, j} Z\right)(x)\right\|_{\mathbb{C}^{d \times d}} \leq g(x)(1+|x|)^{-m_{j}}\|Z\|_{C_{\mathrm{b}}([\tau, \infty))^{d \times d}}
$$


for all $x \in[\tau, \infty)$ and $Z \in C_{\mathrm{b}}([\tau, \infty))^{d \times d}$.

To prove the claims $(a)$ and $(b)$ we use (8.3) and bound the norm of the first integral in (8.4) by the expression

$$
\begin{aligned}
& c\left\|Z_{+}^{(j)}\right\|_{C_{\mathrm{b}}([\tau, \infty))^{d \times d}}(1+|x|)^{-m_{j}} \\
& \quad \times \int_{x}^{\infty} d x^{\prime} \sum_{k=j}^{d^{\prime}} e^{\left(\varkappa_{k}-\varkappa_{j}\right)\left(x-x^{\prime}\right)}\left(1+\left|x-x^{\prime}\right|\right)^{m_{k}}\left(1+\left|x^{\prime}\right|\right)^{m_{j}}\left\|R\left(x^{\prime}\right)\right\|_{\mathbb{C}^{d \times d}} \\
& \leq c\left\|Z_{+}^{(j)}\right\|_{C_{\mathrm{b}}([\tau, \infty))^{d \times d}} \int_{x}^{\infty} d x^{\prime}\left(1+\left|x^{\prime}\right|\right)^{2 m_{j}}\left\|R\left(x^{\prime}\right)\right\|_{\mathbb{C}^{d \times d}},
\end{aligned}
$$

since, due to $x \leq x^{\prime}$, for $k \geq j+1$ in the sum above, we have $\varkappa_{k}-\varkappa_{j}>0$ and $\exp \left(\left(\varkappa_{k}-\varkappa_{j}\right)\left(x-x^{\prime}\right)\right)\left(1+\left|x-x^{\prime}\right|\right)^{m_{k}} \leq c$, while for $k=j$ we have $\left(1+\left|x-x^{\prime}\right|\right)^{m_{j}} \leq$ $c\left(1+\left|x^{\prime}\right|\right)^{m_{j}}$. Similarly, the norm of the second integral in (8.4) is dominated by the expression

$$
\begin{aligned}
& c \| Z_{+}^{(j)} \|_{C_{\mathrm{b}}([\tau, \infty))^{d \times d}}(1+|x|)^{-m_{j}} \\
& \times \int_{\tau}^{x} d x^{\prime} \sum_{k=1}^{j-1} e^{\left(\varkappa_{k}-\varkappa_{j}\right)\left(x-x^{\prime}\right)}\left(1+\left|x-x^{\prime}\right|\right)^{m_{k}}\left(1+\left|x^{\prime}\right|\right)^{m_{j}}\left\|R\left(x^{\prime}\right)\right\|_{\mathbb{C}^{d \times d}} \\
& \leq c\left\|Z_{+}^{(j)}\right\|_{C_{\mathrm{b}}([\tau, \infty)) d \times d} \\
& \quad \times \int_{\tau}^{x} d x^{\prime} \sum_{k=1}^{j-1} e^{\left(\varkappa_{k}-\varkappa_{j}\right)\left(x-x^{\prime}\right) / 2}\left(1+\left|x^{\prime}\right|\right)^{2 m_{j}}\left\|R\left(x^{\prime}\right)\right\|_{\mathbb{C}^{d \times d}}
\end{aligned}
$$

since $\exp \left(\left(\varkappa_{k}-\varkappa_{j}\right)\left(x-x^{\prime}\right) / 2\right)\left[1+\left|x-x^{\prime}\right|\right]^{m_{k}} \leq c$ due to $\varkappa_{k}-\varkappa_{j}<0$ and $x-x^{\prime} \geq 0$. Now condition (8.9) and Lemma 6.4 yield claims $(a)$ and $(b)$.

Using claim $(a)$, assertion $(i)$ follows. Also, the uniqueness part in assertion $(i i)$ holds since (8.4) is a Volterra integral equation for $j=1$. Assertions (7.13), and (iii) in Theorem 7.3, and assertion ( $i v)$ in Theorem 8.3 follow from claim $(b)$ similar to the proof of Theorem 7.3. For instance, to prove $(i i i)$, we note first that claim (b) implies the estimate

$$
\left\|Y_{+}^{(j)}(x)-e^{x A} Q_{j}\right\|_{\mathbb{C}^{d \times d}} \leq c g(x) e^{\varkappa_{j} x}, \quad x \geq \tau, \quad \text { where } \quad \lim _{x \rightarrow \infty} g(x)=0 .
$$

If $y$ is a nonzero $\mathbb{C}^{d}$-valued solution of $(8.2)$ with $y(0) \in \operatorname{ran}\left(Y_{+}^{(j)}(0)\right)$ then, using (8.16), its Lyapunov exponent satisfies the inequality

$$
\begin{aligned}
& \lambda_{+}(y) \leq \max \left\{\limsup _{x \rightarrow \infty} \frac{\log \left\|Y_{+}^{(j)}(x)-e^{x A} Q_{j}\right\|_{\mathbb{C}^{d \times d}}}{x}, \varkappa_{j}\right\}<\varkappa_{j+1}=\lambda_{+}^{\prime}\left(P_{j+1}\right) \\
& \lambda_{+}(y)<0, \quad j=2, \ldots, k_{0}-1 \\
& =k_{0} .
\end{aligned}
$$

Applying Remark 2.7 to (8.2) we conclude that $y(0) \in \operatorname{ran}\left(\sum_{k=1}^{j} P_{k}\right)$ for $j=$ $1, \ldots, k_{0}$. However, for $j=2, \ldots, k_{0}$, the assumption $y(0)=y_{0} \in \operatorname{ran}\left(\sum_{k=1}^{j-1} P_{k}\right)$ leads to a contradiction similar to the proof of Theorem 7.3 (iii). Indeed, using the same notation as in that proof, we have

$$
\left\|e^{x A} Q_{j} y_{1}\right\|_{\mathbb{C}^{d}} \leq c\left\|e^{x A} Q_{j}-Y_{+}^{(j)}(x)\right\|_{\mathbb{C}^{d \times d}}+\|y(x)\|_{\mathbb{C}^{d}} \leq c g(x) e^{\varkappa_{j} x}+c e^{\varkappa_{j-1} x},
$$


where we used (8.16), the assumption $y(0) \in \operatorname{ran}\left(\sum_{k=1}^{j-1} P_{k}\right)$, and Remark 2.7 applied to (8.2). It follows that $e^{-\varkappa_{j} x}\left\|e^{x A} Q_{j} y_{1}\right\|_{\mathbb{C}^{d}} \rightarrow 0$ as $x \rightarrow \infty$, in contradiction with the explicit formula for $e^{\left.x A\right|_{\operatorname{ran}\left(Q_{j}\right)}}$ in terms of the Jordan blocks (cf. [14, Example I.2.5]). This proves assertion (iii). Assertion (iv) follows from (8.16).

It remains to prove assertion $(v)$. For this purpose we consider the truncated perturbed equation, $y^{\prime}(x)=\left(A+R_{n}(x)\right) y(x), x \in \mathbb{R}$, with $R_{n}$ as in (4.14). For a sufficiently large $\tau>0$, we define the operator $F_{\varkappa_{j}, n}^{\tau, j}$ on $C_{\mathrm{b}}([\tau, \infty))^{d \times d}$ by the right-hand side of equation (8.4) with $R$ replaced by $R_{n}$. Since $R_{n} \underset{n \rightarrow \infty}{\rightarrow} R$ in $L^{1}\left(\mathbb{R} ;(1+|x|)^{2 m} d x\right)^{d \times d}$, estimates similar to (8.14) and (8.15) show that $F_{\varkappa_{j}, n}^{\tau, j} \underset{n \rightarrow \infty}{\rightarrow}$ $F_{\varkappa_{j}}^{\tau, j}$ in operator norm on the Banach space $C_{\mathrm{b}}([\tau, \infty))^{d \times d}$. It follows that

$$
Y_{ \pm, n, F}^{(j)}(0) \underset{n \rightarrow \infty}{\rightarrow} Y_{ \pm, F}^{(j)}(0)
$$

where $Y_{ \pm, n, F}^{(j)}$ are the generalized matrix-valued Jost solutions corresponding to the truncated perturbed equation, and $Y_{ \pm}^{(j)}=Y_{ \pm, F}^{(j)}$ are the generalized matrix-valued Jost solutions of (8.2) constructed in part $(i)$ of the current proof. The rest of the proof is similar to the arguments in the proof of Theorem 7.8. Indeed, using the notations introduced in that proof, to establish formula (8.11), we need to show that $\operatorname{det}_{2}(I+K)=e^{\Theta} \operatorname{det}_{\mathbb{C}^{d}}\left(\mathcal{Y}_{+, F}+\mathcal{Y}_{-, F}\right)$. The latter equality follows from (7.38), (7.39), and (7.40). As before, (7.38) follows from Remark 4.3, and we already know from (8.20) that (7.40) holds. Thus, it remains to prove (7.39). For this we will take advantage of the fact that the support of $R_{n}$ is compact (and thus we may drop the improper integral in (8.4) for sufficiently large $x>0$ ). Indeed, we may assume that $n>\tau$. It follows from (4.26) and (7.32) that $Y_{+, n, V}^{(j)}(x)=e^{x A} Q_{j}$ for $x>n$. On the other hand, for $x>n$ we also have (cf. (8.4))

$$
Y_{+, n, F}^{(j)}(x)=e^{x A} Q_{j}+\int_{\tau}^{n} d x^{\prime} e^{\left(x-x^{\prime}\right) A}\left(\sum_{k=1}^{j-1} Q_{k}\right) R\left(x^{\prime}\right) Y_{+, n, F}^{(j)}\left(x^{\prime}\right) .
$$

Thus, using (8.3) for $x>n$ and a sufficiently small $\varepsilon>0$, we obtain the estimate

$$
\begin{aligned}
\left\|Y_{+, n, F}^{(j)}(x)-Y_{+, n, V}^{(j)}(x)\right\|_{\mathbb{C}^{d \times d}} \leq c \int_{\tau}^{n} d x^{\prime} \sum_{k=1}^{j-1} e^{\varkappa_{k}\left(x-x^{\prime}\right)}\left(1+\left|x-x^{\prime}\right|\right)^{m_{k}} \\
\times\left\|R\left(x^{\prime}\right)\right\|_{\mathbb{C}^{d \times d}}\left\|Y_{+, n, F}^{(j)}\left(x^{\prime}\right)\right\|_{\mathbb{C}^{d \times d}} \\
\leq c \int_{\tau}^{n} d x^{\prime} \sum_{k=1}^{j-1} e^{\left(\varkappa_{k}-\varepsilon\right)\left(x-x^{\prime}\right)} \max _{x \geq x^{\prime} \in \mathbb{R}}\left[e^{\left(\varkappa_{k}+\varepsilon\right)\left(x-x^{\prime}\right)}\left(1+\left|x-x^{\prime}\right|\right)^{m_{k}}\right] \\
\quad \times\left\|R\left(x^{\prime}\right)\right\|_{\mathbb{C}^{d \times d}}\left\|Y_{+, n, F}^{(j)}\left(x^{\prime}\right)\right\|_{\mathbb{C}^{d \times d}} \\
\leq c e^{\left(\varkappa_{j}-\varepsilon\right) x} \text { with } c=c(n, \tau),
\end{aligned}
$$

since $\varkappa_{k}<\varkappa_{j}$ for $k=1, \ldots, j-1$. Using Remark 2.7 we therefore obtain the inclusion $\operatorname{ran}\left(Y_{+, n, F}^{(j)}(0)-Y_{+, n, V}^{(j)}(0)\right) \subseteq \operatorname{ran}\left(\sum_{k=1}^{j-1} P_{k}\right), j=2, \ldots, k_{0}$. Now (7.39) follows as in the proof of Lemma 7.6 since $Y_{+, n, F}^{(j)}$ and $Y_{+, n, V}^{(j)}$ possess all properties (ii)-(iv) in Theorem 7.3 (see Remark 7.7). This concludes the proof of assertion $(v)$ in Theorem 8.3. 
Remark 8.4. We emphasize that although the generalized matrix-valued Jost solutions in the sense of Definition 8.2 are not uniquely determined and depend on the choice of $\tau$ in (8.4) and (8.5), formula (8.11) shows that the Evans determinant $D$ is independent of the choice of the solutions and hence independent of $\tau$.

Remark 8.5. We note that Theorem 8.3 (iv) also follows from the Levinson theorem (cf. Remark 7.11) for asymptotically constant coefficients under the additional assumption that the spectrum of $A$ is simple. In this context we refer to [13, Theorem 1.8.1] and also to [13, Sect. 1.10] for additional results on the asymptotic behavior of solutions of (1.2) with $A$ of Jordan block-type.

\section{The Evans Function}

In this section we relate the Evans determinant $D$ introduced in Definition 7.5 to the Evans function, E. First, we recall one of many equivalent definitions of the Evans function available in the literature (see, e.g., $[1,5,35]$ ), namely, the definition using exponential dichotomies, see [57, Definition 4.1]. Consider a family of differential equations

$$
y^{\prime}(x)=B(z, x) y(x), \quad x \in \mathbb{R},
$$

parameterized by a complex spectral parameter $z \in \Omega$, where $\Omega \subseteq \mathbb{C}$ is open and simply connected. It is assumed that the locally integrable $\mathbb{C}^{d \times d}$-valued functions $B(z, \cdot)$ depend on $z \in \Omega$ analytically. Since in this paper we are not concerned ${ }^{3}$ with function theoretic issues related to the Evans function such as its analytic continuations, etc., we will fix a value $z_{0} \in \Omega$ in what follows, and will give the definition of $E=E\left(z_{0}\right)$ for this $z_{0} \in \Omega$. Accordingly, we will suppress the $z$ dependence in the notations for the differential equations above, and consider just one differential equation $y^{\prime}(x)=B(x) y(x)$.

Hypothesis 9.1. Assume that the differential equation $y^{\prime}(x)=B(x) y(x), x \in \mathbb{R}$, has an exponentially bounded propagator on $\mathbb{R}$ and, in addition, an exponential dichotomy $P_{+}$on $\mathbb{R}_{+}$and an exponential dichotomy $P_{-}$on $\mathbb{R}_{-}$. Moreover, assume that the ranks of the projections $P_{+}$and $P_{-}$are equal, and denote the common value by $n$ so that

$$
n=\operatorname{dim}\left(\operatorname{ran}\left(P_{+}\right)\right)=\operatorname{dim}\left(\operatorname{ran}\left(P_{-}\right)\right) \text {and } d-n=\operatorname{dim}\left(\operatorname{ker}\left(P_{+}\right)\right)=\operatorname{dim}\left(\operatorname{ker}\left(P_{-}\right)\right) .
$$

Definition 9.2. Assume Hypothesis 9.1. Choose any vector basis $\left\{\mathrm{e}_{1}, \ldots, \mathrm{e}_{n}\right\}$ of the subspace $\operatorname{ran}\left(P_{+}\right)$, and any vector basis $\left\{\mathrm{e}_{n+1}, \ldots, \mathrm{e}_{d}\right\}$ of the subspace $\operatorname{ker}\left(P_{-}\right)$. The Evans function, $E$, is then defined as the determinant of the $d \times d$ matrix with columns $\mathrm{e}_{k}, k=1, \ldots, d$.

Clearly, $E=0$ if and only if the subspaces $\operatorname{ran}\left(P_{+}\right)$and $\operatorname{ker}\left(P_{-}\right)$have a nonzero intersection. $\mathbb{C}^{d}$-valued solutions $y_{+}^{(k)}$ on $\mathbb{R}_{+}$with initial data $y_{+}^{(k)}(0)=\mathrm{e}_{k}, k=$ $1, \ldots, n$, form a basis in the subspace of all bounded solutions of the differential equation $y^{\prime}(x)=B(x) y(x), x \in \mathbb{R}_{+}$. Similarly, $\mathbb{C}^{d}$-valued solutions $y_{-}^{(k)}$ on $\mathbb{R}_{-}$ with initial data $y_{-}^{(k)}(0)=\mathrm{e}_{k}, k=n+1, \ldots, d$, form a basis in the subspace of all solutions bounded on $\mathbb{R}_{-}$. Thus, $E=0$ if and only if the differential equation has a $\mathbb{C}^{d}$-valued solution bounded on $\mathbb{R}$; see the Introduction. We emphasize, that

\footnotetext{
${ }^{3}$ See, however, Remark 9.5 below.
} 
the Evans function $E$ just defined is not unique, and depends on the choice of the vectors $\mathrm{e}_{k}, k=1, \ldots, d$. Moreover, this definition does not assume any perturbation structure in the differential equation, and, in particular, can be used to define the Evans functions for both (1.1) and (1.2).

Returning to the principal theme of the current paper, we will set $B(x)=A(x)+$ $R(x), x \in \mathbb{R}$, with the matrix-valued functions $A$ and $R$ as in (1.1) and (1.2). Next, we intend to show that, in fact, $D=E$, where $D$ is the Evans determinant in Definition 7.5, and $E$ is the Evans function defined using a special choice of the vectors $\mathrm{e}_{k}, k=1, \ldots, d$, given by the initial data of the generalized matrix-valued Jost solutions of (1.2).

For this, we assume in accordance with Hypothesis 2.8, that the unperturbed $d \times d$ matrix differential equation $y^{\prime}(x)=A(x) y(x), x \in \mathbb{R}$, with $A \in L_{\text {loc }}^{1}(\mathbb{R})^{d \times d}$, has an exponentially bounded propagator and an exponential dichotomy $Q$ on $\mathbb{R}$. Assume, in addition (see Hypothesis 7.1), that the unperturbed equation (1.1) has an exponential splitting of order $d^{\prime}, 2 \leq d^{\prime} \leq d$, so that $\mathbb{C}^{d}=\operatorname{ran}\left(Q_{1}\right) \dot{+} \cdots \dot{+} \operatorname{ran}\left(Q_{d^{\prime}}\right)$, and that the Bohl segments corresponding to the projections $Q_{j}$ are disjoint. We also have $Q=\sum_{j=1}^{k_{0}} Q_{j}$ and $I-Q=\sum_{j=k_{0}+1}^{d^{\prime}} Q_{j}$ for some $1<k_{0} \leq d^{\prime}$. Suppose that a $d \times d$ matrix-valued function $R$ is such that $\|R\|_{\mathbb{C}^{d \times d}} \in L^{1}(\mathbb{R})$. Under these assumptions, due to Lemma 2.13 and its analog for $\mathbb{R}_{-}$, we conclude that the perturbed equation $y^{\prime}(x)=(A(x)+R(x)) y(x), x \in \mathbb{R}_{ \pm}$, has an exponential dichotomy $P_{+}$on $\mathbb{R}_{+}$and an exponential dichotomy $P_{-}$on $\mathbb{R}_{-}$such that $\operatorname{dim}(\operatorname{ran}(Q))=\operatorname{dim}\left(\operatorname{ran}\left(P_{+}\right)\right)$and $\operatorname{dim}(\operatorname{ker}(Q))=\operatorname{dim}\left(\operatorname{ker}\left(P_{-}\right)\right)$. Thus, (9.2) holds if we let

$$
n=\operatorname{dim}(\operatorname{ran}(Q))=\sum_{j=1}^{k_{0}} \operatorname{dim}\left(\operatorname{ran}\left(Q_{j}\right)\right) .
$$

The same conclusions hold provided the unperturbed equation (1.1) is autonomous, and we assume Hypothesis 8.1. We summarize our assumptions as follows.

Hypothesis 9.3. For the unperturbed equation (1.1), respectively, (8.1) assume Hypotheses 2.8 and 7.1, respectively, Hypothesis 8.1. Also, assume that $\|R\|_{\mathbb{C}^{d \times d}} \in$ $L^{1}(\mathbb{R})$. In addition, assume that the perturbed equation (1.2), respectively, (8.2) has a system of generalized matrix-valued Jost solutions $Y_{+}^{(j)}, j=1, \ldots, k_{0}$, on $\mathbb{R}_{+}$ and $Y_{-}^{(j)}, j=k_{0}+1, \ldots, d^{\prime}$, on $\mathbb{R}_{-}$in the sense of Definition 7.2, respectively, in the sense of Definition 8.2.

Our final result then reads as follows.

Theorem 9.4. Assume Hypotheses 9.3. Define the matrices $\mathcal{Y}_{ \pm}$and the Evans determinant $D$ as indicated in Definition 7.5. In addition, with $n$ as in (9.3), let $\mathrm{e}_{k}$, $k=1, \ldots, n$, denote the nonzero columns of the matrix $\mathcal{Y}_{+}$and $\mathrm{e}_{k}, k=n+1, \ldots, d$, denote the nonzero columns of the matrix $\mathcal{Y}_{-}$. Letting $B(x)=A(x)+R(x), x \in \mathbb{R}$, and using the vectors $\mathrm{e}_{k}, k=1, \ldots, d$, define the Evans function $E$ for the perturbed equation (1.2) as indicated in Definition 9.2. Then

$$
E=D .
$$

Moreover, if, in addition, the perturbation $R$ satisfies the assumptions in Theorem 7.8, respectively, Theorem 8.3, then the 2-modified perturbation determinant $\operatorname{det}_{2}(I+K)$ admits the representation

$$
\operatorname{det}_{2}(I+K)=e^{\Theta} E,
$$


where $\Theta$ is defined in (4.3), respectively, in (8.12).

Proof. We claim that $n$ nonzero columns $y_{k}(x)$ of the $d \times d$ matrix $\mathcal{Y}_{+}(x)=$ $\sum_{j=1}^{k_{0}} Y_{+}^{(j)}(x), x \geq 0$, provide a basis in the space of all bounded $\mathbb{C}^{d}$-valued solutions of the perturbed equation (1.2) on $\mathbb{R}_{+}$, while $d-n$ nonzero columns $y_{k}(x)$ of the matrix $\mathcal{Y}_{-}(x)=\sum_{j=k_{0}+1}^{d^{\prime}} Y_{-}^{(j)}(x), x \leq 0$, yield a basis in the space of all bounded $\mathbb{C}^{d}$-valued solutions of the perturbed equation $y^{\prime}(x)=(A(x)+R(x)) y(x)$ on $\mathbb{R}_{-}$. Indeed, the claim follows from (7.3) and (7.5) (or, for the autonomous unperturbed equation (1.1), from assertion $(i v)$ in Theorem 8.3) and the fact that the nonzero columns of the matrix $\Phi(x) Q$, respectively, $\Phi(x)\left(I_{d}-Q\right)$, provide a basis in the space of all bounded solutions of the unperturbed equation (1.1) on $\mathbb{R}_{+}$, respectively, $\mathbb{R}_{-}$.

As a result, letting $\mathrm{e}_{k}=y_{k}(0), k=1, \ldots, d$, we selected a basis $\mathrm{e}_{1}, \ldots, \mathrm{e}_{n}$ in the subspace $\operatorname{ran}\left(P_{+}\right)$, and a basis $\mathrm{e}_{n+1}, \ldots, \mathrm{e}_{d}$ in the subspace $\operatorname{ker}\left(P_{-}\right)$needed for the definition of the Evans function $E$ as above. In particular, this proves that the Evans determinant $D$ equals the Evans function $E$ defined with this choice of bases. The result in Theorem 7.8 (or, for the autonomous unperturbed equation (1.1), Theorem $8.3(v)$ ) now states that, under additional assumptions on $R$ (see (7.11) and (7.12) or (8.9)), the 2-modified perturbation determinant $\operatorname{det}_{2}(I+K)$ admits the representation (9.5).

Remark 9.5. Assume that the matrix-valued functions $A(z, \cdot)$ and $R(z, \cdot)$ in $(1.2)$ analytically depend on a spectral parameter $z \in \Omega \subseteq \mathbb{C}$. Moreover, assume that the equation $y^{\prime}(x)=A(x, z) y(x), x \in \mathbb{R}$, has an exponential dichotomy on $\mathbb{R}$ for each $z \in \Omega$. Then the dichotomy projection $Q=Q(z)$ for (1.1) on $\mathbb{R}$ is an

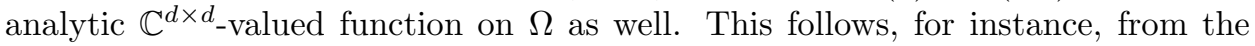
discussion in Remark 2.11 (alternatively, see [53] or [57, p. 995]). Thus, $\Theta(z)$ in (4.3) is an analytic function for $z \in \Omega$. Moreover, the corresponding function $K(z)$ is also analytic as a function with values in the space of Hilbert-Schmidt operators equipped with the $\mathcal{B}\left(L^{2}(\mathbb{R})^{d}\right)$-norm. Hence, the 2 -modified perturbation determinant $\operatorname{det}_{2}(I+K(z))$ is also analytic for $z \in \Omega$. As a final result, it follows from formula (7.31) in Theorem 7.8 (or from formula (8.11) in Theorem 8.3) that the Evans determinant $D(z)$ also depends on $z \in \Omega$ analytically. In view of the discussion in this section we just proved that the Evans function $E(z)$ is an analytic function of the spectral parameter $z \in \Omega$ (cf. also [1] for an entirely different proof of this result).

Remark 9.6. The assertions in Lemma 7.9 and Corollary 7.10 (which are more general than Lemma 7.6 and Theorem 7.8) can be used in the context of this section as follows. Let $\mathrm{e}_{i}^{(j)}, i=1, \ldots, \operatorname{dim}\left(\operatorname{ran}\left(Q_{j}\right)\right)$, denote an arbitrary basis in $\operatorname{ran}\left(Q_{j}\right)$, $j=1, \ldots, d^{\prime}$, and let $N$ denote the $d \times d$ matrix with the columns $\mathrm{e}_{i}^{(j)}$. For brevity, we enumerate the columns as $\mathrm{e}_{k}, k=1, \ldots, d$. We note that $\left\{\mathrm{e}_{k}\right\}_{k=1}^{d}$ is a basis in $\mathbb{C}^{d}$. Passing from the standard basis in $\mathbb{C}^{d}$ (i.e., $\left.(1,0, \ldots, 0), \ldots,(0, \ldots, 0,1)\right)$ to this new basis, we observe that for each $j=1, \ldots, d^{\prime}$, the matrix $\left(q_{\ell k}^{(j)}\right)_{1 \leq \ell, k \leq d}$ corresponding to the operator $Q_{j}$ in the basis $\left\{\mathrm{e}_{k}\right\}_{k=1}^{d}$ is a diagonal matrix with all entries being equal to zero except the entries $q_{\ell \ell}^{(j)}=1$ for $\ell=n_{j-1}+1, \ldots, n_{j}$. Here we denote $n_{0}=0$ and $n_{j}=\sum_{k=1}^{j} \operatorname{dim}\left(\operatorname{ran}\left(Q_{k}\right)\right)$ for $j=1, \ldots, d^{\prime}$. Next, we introduce the $\mathbb{C}^{d}$-valued solutions of the unperturbed equation (1.1) by $y_{k}(x)=\Phi(x) \mathrm{e}_{k}, x \in \mathbb{R}$, 
$k=1, \ldots, d$. Let $\tilde{y}_{k}(x), x \in \mathbb{R}, k=1, \ldots, d$, be (not necessarily unique) solutions of the perturbed equation (1.2) that satisfy the asymptotic conditions

$$
\begin{aligned}
& \widetilde{y}_{k}(x) \underset{x \rightarrow \infty}{=} y_{k}(x)+o\left(e^{\varkappa_{+}^{\prime}(Q) x}\right), \quad k=1, \ldots, n, \\
& \widetilde{y}_{k}(x) \underset{x \rightarrow-\infty}{=}=y_{k}(x)+o\left(e^{\varkappa_{-}\left(I_{d}-Q\right) x}\right), \quad k=n+1, \ldots, d,
\end{aligned}
$$

where $n=\operatorname{dim}(\operatorname{ran}(Q))$. Now consider two Evans functions, $E_{\left\{\tilde{y}_{k}\right\}}$ and $E_{\left\{y_{k}\right\}}$ for the perturbed and unperturbed equations, (1.2) and (1.1), respectively. Here, $E_{\left\{y_{k}\right\}}=\operatorname{det}_{\mathbb{C}^{d}}(N)$, and $E_{\left\{\widetilde{y}_{k}\right\}}=\operatorname{det}_{\mathbb{C}^{d}}(\widetilde{N})$ with the matrix $\widetilde{N}$ defined in $(7.47)$ and constructed using the matrices $N_{j}=N Q_{j}=Q_{j} N, j=1, \ldots, d^{\prime}$. The representation

$$
\operatorname{det}_{2}(I+K)=e^{\Theta} \frac{E_{\left\{\widetilde{y}_{k}\right\}}}{E_{\left\{y_{k}\right\}}}
$$

now follows from Corollary 7.10.

\section{Appendix A. Operators with Semi-Separable Kernels}

In this appendix we recall some of the results derived in [28]. Let $d_{1}, d_{2} \in \mathbb{N}$, $d=d_{1}+d_{2}$, and let $f_{j}$ and $g_{j}, j=1,2$, be given matrix-valued functions on $\mathbb{R}$ satisfying

$$
f_{j} \in L^{2}(\mathbb{R})^{d \times d_{j}}, \quad g_{j} \in L^{2}(\mathbb{R})^{d_{j} \times d}, \quad j=1,2 .
$$

On the space $L^{2}(\mathbb{R})^{d}$ we consider the Hilbert-Schmidt integral operator defined by

$$
(K u)(x)=\int_{\mathbb{R}} d x^{\prime} K\left(x, x^{\prime}\right) u\left(x^{\prime}\right), \quad u \in L^{2}(\mathbb{R})^{d},
$$

where $K(\cdot, \cdot)$ is a semi-separable $d \times d$ matrix-valued integral kernel defined by

$$
K\left(x, x^{\prime}\right)=\left\{\begin{array}{ll}
f_{1}(x) g_{1}\left(x^{\prime}\right), & x \geq x^{\prime}, \\
f_{2}(x) g_{2}\left(x^{\prime}\right), & x<x^{\prime},
\end{array} \quad x, x^{\prime} \in \mathbb{R} .\right.
$$

In addition, we introduce the $d \times d$ matrix-valued integral kernel

$$
H\left(x, x^{\prime}\right)=f_{1}(x) g_{1}\left(x^{\prime}\right)-f_{2}(x) g_{2}\left(x^{\prime}\right),
$$

and the corresponding Volterra integral equations

$$
\begin{aligned}
& \hat{f}_{1}(x)=f_{1}(x)-\int_{x}^{\infty} d x^{\prime} H\left(x, x^{\prime}\right) \hat{f}_{1}\left(x^{\prime}\right), \\
& \hat{f}_{2}(x)=f_{2}(x)+\int_{-\infty}^{x} d x^{\prime} H\left(x, x^{\prime}\right) \hat{f}_{2}\left(x^{\prime}\right) .
\end{aligned}
$$

We note that equations (A.5) and (A.6) have a unique pair of solutions satisfying $\hat{f}_{j} \in L^{2}(\mathbb{R})^{d \times d_{j}}, j=1,2$. Finally, we introduce the $d \times d$ matrix-valued function $B$ by

$$
B(x)=\left(\begin{array}{cc}
g_{1}(x) f_{1}(x) & g_{1}(x) f_{2}(x) \\
-g_{2}(x) f_{1}(x) & -g_{2}(x) f_{2}(x)
\end{array}\right), \quad x \in \mathbb{R} .
$$

Theorem A.1 ([28]). Assume (A.1). Then:

(i) The first-order $d \times d$ matrix differential equation

$$
U^{\prime}(x)=B(x) U(x), \quad x \in \mathbb{R},
$$


permits an explicit particular solution given by the formula

$$
U(x)=\left(\begin{array}{cc}
I_{d_{1}}-\int_{x}^{\infty} d x^{\prime} g_{1}\left(x^{\prime}\right) \hat{f}_{1}\left(x^{\prime}\right) & \int_{-\infty}^{x} d x^{\prime} g_{1}\left(x^{\prime}\right) \hat{f}_{2}\left(x^{\prime}\right) \\
\int_{x}^{\infty} d x^{\prime} g_{2}\left(x^{\prime}\right) \hat{f}_{1}\left(x^{\prime}\right) & I_{d_{2}}-\int_{-\infty}^{x} d x^{\prime} g_{2}\left(x^{\prime}\right) \hat{f}_{2}\left(x^{\prime}\right)
\end{array}\right), \quad x \in \mathbb{R} .
$$

(ii) The modified Fredholm determinant $\operatorname{det}_{2}(I-K)$ has the following representation:

$$
\begin{aligned}
\operatorname{det}_{2}(I-K) & =\operatorname{det}_{\mathbb{C}^{n}}(U(-\infty)) \exp \left(\int_{\mathbb{R}} d x \operatorname{tr}_{\mathbb{C}^{m}}\left(f_{1}(x) g_{1}(x)\right)\right) \\
& =\operatorname{det}_{\mathbb{C}^{n}}(U(\infty)) \exp \left(\int_{\mathbb{R}} d x \operatorname{tr}_{\mathbb{C}^{m}}\left(f_{2}(x) g_{2}(x)\right)\right)
\end{aligned}
$$
$\mathbb{R}$

By Liouville's formula (cf., e.g., [33, Theorem IV.1.2]) one infers for any $x, x_{0} \in$

$$
\operatorname{det}_{\mathbb{C}^{d}}(U(x))=\operatorname{det}_{\mathbb{C}^{d}}\left(U\left(x_{0}\right)\right) \exp \left(\int_{x_{0}}^{x} d x^{\prime} \operatorname{tr}_{\mathbb{C}^{d}}\left(B\left(x^{\prime}\right)\right)\right)
$$

and thus, for any $x_{0} \in \mathbb{R}$, one has from Theorem A.1 $(i i)$,

$$
\begin{aligned}
\operatorname{det}_{2}(I-K)= & \operatorname{det}_{\mathbb{C}^{d}}(U(\infty)) \exp \left(\int_{\mathbb{R}} d x \operatorname{tr}_{\mathbb{C}^{d}}\left(f_{2}(x) g_{2}(x)\right)\right) \\
= & \operatorname{det}_{\mathbb{C}^{d}}\left(U\left(x_{0}\right)\right) \exp \left(\int_{x_{0}}^{\infty} d x \operatorname{tr}_{\mathbb{C}^{d}}\left(g_{1}(x) f_{1}(x)-g_{2}(x) f_{2}(x)\right)\right) \\
& \times \exp \left(\int_{\mathbb{R}} d x \operatorname{tr}_{\mathbb{C}^{d}}\left(f_{2}(x) g_{2}(x)\right)\right) .
\end{aligned}
$$

This results in the representation

$$
\begin{aligned}
& \operatorname{det}_{2}(I-K)=\operatorname{det}_{\mathbb{C}^{d}}\left(U\left(x_{0}\right)\right) \exp ( \int_{-\infty}^{x_{0}} d x \operatorname{tr}_{\mathbb{C}^{d}}\left(f_{2}(x) g_{2}(x)\right) \\
&\left.+\int_{x_{0}}^{\infty} d x \operatorname{tr}_{\mathbb{C}^{d}}\left(f_{1}(x) g_{1}(x)\right)\right) \\
&=\operatorname{det}_{\mathbb{C}^{d}}\left(U\left(x_{0}\right)\right) \exp \left(\operatorname { t r } _ { \mathbb { C } ^ { d } } \left(\int_{-\infty}^{x_{0}} d x K(x-0, x)\right.\right. \\
&\left.\left.+\int_{x_{0}}^{\infty} d x K(x+0, x)\right)\right) .
\end{aligned}
$$

We note that if $K$ is a trace class operator with a continuous integral kernel, then $\operatorname{tr}_{\mathbb{C}^{d}}(B(x))=0$, and applying [12, p. 1086-87] the Fredholm determinant can be represented in the simpler form

$$
\operatorname{det}(I-K)=\operatorname{det}_{\mathbb{C}^{d}}\left(U\left(x_{0}\right)\right)=\operatorname{det}_{\mathbb{C}^{d}}(U(-\infty))=\operatorname{det}_{\mathbb{C}^{d}}(U(\infty))
$$

Appendix B. The Proof of Lemmas 2.13 and 2.14

Proof of Lemma 2.13. Using rescaling (see Remark 2.2), we will assume without loss of generality that $\varkappa_{+}(Q)=-\varkappa_{+}^{\prime}\left(I_{d}-Q\right)$. We choose $\varkappa$ so that $0<\varkappa<$ $-\varkappa_{+}(Q)$. We claim that:

(i) $\operatorname{dim} N=\operatorname{dim}(\operatorname{ran}(Q))$,

and

(ii) for a constant $C(\varkappa)>0$ solutions $y$ of (1.2) satisfy:

$$
\|y(x)\|_{\mathbb{C}^{d}} \leq C(\varkappa) e^{-\varkappa\left(x-x^{\prime}\right)}\left\|y\left(x^{\prime}\right)\right\|_{\mathbb{C}^{d}}, \quad x \geq x^{\prime} \geq 0, \text { if } y(0) \in N
$$




$$
\|y(x)\|_{\mathbb{C}^{d}} \leq C(\varkappa) e^{\varkappa\left(x-x^{\prime}\right)}\left\|y\left(x^{\prime}\right)\right\|_{\mathbb{C}^{d}}, \quad x^{\prime} \geq x \geq 0, \text { if } y(0) \in \operatorname{ker}(Q) .
$$

As soon as these claims are proved, the assertions in the lemma follow. Indeed, since $\mathbb{C}^{d}=N \oplus \operatorname{ker}(Q)$ by $(i)$ and (B.1)-(B.2), the exponential boundedness of the propagator of (1.1) implies that $P$ is an exponential dichotomy for (1.2) by the proof of Lemma IV.3.2 in [10]. (We note that in the proof of this lemma only the exponential boundedness of the propagator has been used.) Since $-\varkappa$ can be chosen arbitrary close to $\varkappa_{+}(Q)$, it follows from (B.1) and the definition of $\varkappa_{+}(P)$ (cf. (2.5)), that $\varkappa_{+}(P) \leq \varkappa_{+}(Q)$. Considering (1.1) as an $L^{1}$-perturbation of (1.2), we obtain the opposite inequality; the second equality in (2.31) is proved similarly using (B.2).

Turning to the proof of the claim, let $\tau>0$, and denote by $F^{\tau}$ the integral operator on $C_{\mathrm{b}}([\tau, \infty))^{d}$ with the integral kernel $F\left(x, x^{\prime}\right) R\left(x^{\prime}\right)$ for $x, x^{\prime} \geq \tau$, where $F\left(x, x^{\prime}\right)$ is defined in (2.30). By a direct estimate, inequalities (2.12) and condition $\|R\|_{\mathbb{C}^{d \times d}} \in L^{1}\left(\mathbb{R}_{+}\right)$imply that $F^{\tau}$ is a contraction on $C_{\mathrm{b}}([\tau, \infty))^{d}$ for $\tau$ sufficiently large. Thus, the formula

$$
y(x)=\Phi(x) q+\left(F^{\tau} y\right)(x), \quad q \in \operatorname{ran}(Q), \quad x \in[\tau, \infty),
$$

gives a one-to-one correspondence between the solutions $y, y(0) \in N$, of (1.2) bounded on $\mathbb{R}_{+}$, and the solutions $\Phi q, q \in \operatorname{ker}(Q)$, of (1.1) bounded on $\mathbb{R}_{+}$. In particular, $(i)$ in the claim above holds. Also, due to (B.3), the bounded solutions $y$ of (1.2) admit the representation (cf. [10, p. 181])

$$
y(x)=\Phi(x) Q \Phi\left(x^{\prime}\right)^{-1} y\left(x^{\prime}\right)+\left(F^{x^{\prime}} y\right)\left(x^{\prime}\right), \quad x \geq x^{\prime} \geq \tau .
$$

Taking $\varepsilon>0$ such that $\alpha=-\varkappa_{+}(Q)-\varepsilon>\varkappa$, and applying the exponential dichotomy estimates in the right-hand side of (B.4), we arrive at the integral inequality

$$
\|y(x)\|_{\mathbb{C}^{d}} \leq a(\varepsilon) e^{-\alpha\left(x-x^{\prime}\right)}\left\|y\left(x^{\prime}\right)\right\|_{\mathbb{C}^{d}}+c(\varepsilon) \int_{x^{\prime}}^{\infty} d s e^{-\alpha|x-s|}\|R(s)\|_{\mathbb{C}^{d \times d}}\|y(s)\|_{\mathbb{C}^{d}} .
$$

Thus, the function $u(x)=e^{\varkappa x}\|y(x)\|_{\mathbb{C}^{d}}, x \geq x^{\prime} \geq \tau$, satisfies the inequality

$$
u(x) \leq a(\varepsilon) e^{-\alpha\left(x-x^{\prime}\right)+\varkappa x}\left\|y\left(x^{\prime}\right)\right\|_{\mathbb{C}^{d}}+c(\varepsilon) \int_{x^{\prime}}^{\infty} d s e^{-\alpha|x-s|+\varkappa(x-s)}\|R(s)\|_{\mathbb{C}^{d \times d}} u(s) .
$$

Claim (B.1) can now be proved using arguments in [10, Sect. III.2]. Indeed, on the space $C_{\mathrm{b}}([\tau, \infty))$ we consider the integral operator $T$ with integral kernel

$$
T\left(x, x^{\prime}\right)=c(\varepsilon) e^{-\alpha\left|x-x^{\prime}\right|+\varkappa\left(x-x^{\prime}\right)}\left\|R\left(x^{\prime}\right)\right\|_{\mathbb{C}^{d \times d}}, \quad x \geq x^{\prime} \geq \tau .
$$

Using equation (2.15) in [10, Sect. III.2] one then shows that $\|T\|$ is bounded by the expression

$$
c(\varepsilon)\left[1+\left(1-e^{-(\alpha-\varkappa)}\right)^{-1}+\left(1-e^{-(\alpha+\varkappa)}\right)^{-1}\right] \sup _{x \in[\tau, \infty)} \int_{x}^{x+1} d s\|R(s)\|_{\mathbb{C}^{d \times d}} .
$$

Thus, the operator $T$ is a contraction for $\tau=\tau(\varkappa, \varepsilon)>0$ suficiently large. Passing to equality in the inequality (B.6), solving the resulting integral equation for $u$ by applying $(I-T)^{-1}$, and using [10, Lemma III.2.1], we derive (B.1) from the estimate

$$
u(x) \leq a(\varepsilon)(1-\|T\|)^{-1} e^{\varkappa x^{\prime}}\left\|y\left(x^{\prime}\right)\right\|_{\mathbb{C}^{d}}, \quad x \geq x^{\prime} \geq \tau .
$$


Turning to the proof of (B.2), we note that any solution $y$ of (1.2) on $\mathbb{R}_{+}$with $y(0) \in \operatorname{ker}(Q)$ solves the equation

$$
\begin{aligned}
y(x)= & \Phi(x)\left(I_{d}-Q\right) \Phi\left(x^{\prime}\right)^{-1} y\left(x^{\prime}\right)+\int_{0}^{x} d s \Phi(x) Q \Phi(s)^{-1} R(s) y(s) \\
& -\int_{x}^{x^{\prime}} d s \Phi(x)\left(I_{d}-Q\right) \Phi(s)^{-1} R(s) y(s), \quad 0 \leq x \leq x^{\prime},
\end{aligned}
$$

which yields the integral inequality

$$
\begin{aligned}
\|y(x)\|_{\mathbb{C}^{d}} \leq & a(\varepsilon) e^{\alpha\left(x-x^{\prime}\right)}\left\|y\left(x^{\prime}\right)\right\|_{\mathbb{C}^{d}}+c(\varepsilon) \int_{0}^{x^{\prime}} d s e^{\alpha(x-s)}\|R(s)\|_{\mathbb{C}^{d \times d}}\|y(s)\|_{\mathbb{C}^{d}} \\
& +c(\varepsilon) \int_{x}^{x^{\prime}} d s e^{-\alpha(x-s)}\|R(s)\|_{\mathbb{C}^{d \times d}}\|y(s)\|_{\mathbb{C}^{d}} \\
= & a(\varepsilon) e^{\alpha\left(x-x^{\prime}\right)}\left\|y\left(x^{\prime}\right)\right\|_{\mathbb{C}^{d \times d}}+c(\varepsilon) \int_{0}^{x^{\prime}} d s e^{-\alpha|x-s|}\|R(s)\|_{\mathbb{C}^{d \times d}}\|y(s)\|_{\mathbb{C}^{d}} \\
& 0 \leq x \leq x^{\prime} . \quad \text { B.11) }
\end{aligned}
$$

Arguments similar to the proof of (B.1) conclude the proof of Lemma 2.13.

Proof of Lemma 2.14. We will use the rescaling from Remark 2.2 as follows: For each $j=1, \ldots, d^{\prime}-1$ we fix $\mu_{j} \in\left(\varkappa_{+}\left(Q_{j}\right), \varkappa_{+}^{\prime}\left(Q_{j+1}\right)\right)$, and for $j=d^{\prime}$ we fix $\mu_{d^{\prime}}>$ $\varkappa_{+}\left(Q_{d}\right)$. Then, for each $j=1, \ldots, d^{\prime}$, the rescaled unperturbed equation $(2.11)$ with $\mu=\mu_{j}$ has an exponential dichotomy on $\mathbb{R}_{+}$with dichotomy projection $\mathcal{Q}_{j}=$ $\sum_{k=1}^{j} Q_{k}$. By Lemma 2.13, the rescaled perturbed equation, $y^{\prime}(x)=(A(x)+R(x)-$ $\left.\mu_{j} I_{d}\right) y(x), x \in \mathbb{R}$, has an exponential dichotomy $\mathcal{P}_{j}$, where $\mathcal{P}_{j}$ is the projection parallel to $\operatorname{ker}\left(\mathcal{Q}_{j}\right)$ on the subspace $N_{j}$ consisting of the values $y(0)$ of the bounded solutions of the rescaled perturbed equation on $\mathbb{R}_{+}$. We note that $\mathcal{P}_{d^{\prime}}=I_{d}$ and set $\mathcal{P}_{0}=0$. Letting $P_{j}=\mathcal{P}_{j}-\mathcal{P}_{j-1}, j=1, \ldots, d^{\prime}$, finishes the proof of Lemma 2.14 .

Acknowledgments. Fritz Gesztesy and Yuri Latushkin gratefully acknowledge a research leave for the academic year 2005/06 granted by the Research Council and the Office of Research of the University of Missouri-Columbia. Moreover, Yuri Latushkin gratefully acknowledges support by the Research Board of the University of Missouri.

\section{REFERENCES}

[1] J. Alexander, R. Gardner, and C. Jones, A topological invariant arising in the stability analysis of travelling waves, J. reine angew. Math. 410, 167-212 (1990).

[2] J. C. Alexander, M. G. Grillakis, C. K. R. T. Jones, and B. Sandstede, Stabiity of pulses on optical fibers with phase-sensitive amplifiers, Z. angew. Math. Phys. 48, 175-192 (1997).

[3] A. Ben-Artzi and I. Gohberg, Dichotomy of systems and invertibility of linear ordinary differential operators, in Time-variant Systems and Interpolation, I. Gohberg (ed.), Operator Theory: Adv. Appl., Vol. 56, Birkhäuser, Basel, 1992, pp. 90-119.

[4] S. Benzoni-Gavage, D. Serre, and K. Zumbrun, Alternate Evans functions and viscous shock waves, SIAM J. Math. Anal. 32, 929-962 (2001).

[5] T. J. Bridges and G. Derks, The symplectic Evans matrix, and the instability of solitary waves and fronts, Arch. Ration. Mech. Anal. 156, 1-87 (2001).

[6] K. Chadan and P. C. Sabatier, Inverse Problems in Quantum Scattering Theory, 2nd ed., Springer, New York, 1989. 
[7] C. Chicone and Y. Latushkin, Evolution Semigroups in Dynamical Systems and Differential Equations, Math. Surv. Monogr. 70, Amer. Math. Soc., Providence, RI, 1999.

[8] W. A. Coppel, Dichotomies in Stability Theory, Lecture Notes in Math. 629, Springer, Berlin, 1978.

[9] D. Cramer and Y. Latushkin, Fredholm determinants and the Evans function for difference equations, Banach Center Publications, to appear.

[10] Ju. Daleckii and M. Krein, Stability of Solutions of Differential Equations in Banach Space, Amer. Math. Soc., Providence, RI, 1974.

[11] J. Deng and S. Nii, Infinite-dimensional Evans function theorey for elliptic eigenvalue problems in a channel, preprint, 2003.

[12] N. Dunford and J. T. Schwartz, Linear Operators Part II: Spectral Theory, Interscience, New York, 1988.

[13] M. S. P. Eastham, The Asymptotic Solution of Linear Differential Systems. Applications of the Levinson Theorem, Oxford Univ. Press, Oxford, 1989.

[14] K. J. Engel and R. Nagel, One-Parameter Semigroups for Linear Evolution Equations, Springer-Verlag, Heidelberg, Berlin, 1999.

[15] J. W. Evans, Nerve axon equations. I. Linear approximations, Indiana Univ. Math. J. 21, 877-885 (1972).

[16] J. W. Evans, Nerve axon equations. II. Stability at rest, Indiana Univ. Math. J. 22, 75-90 (1972). Errata: Indiana Univ. Math. J. 25, 301 (1976).

[17] J. W. Evans, Nerve axon equations. III: Stability of the nerve impulse, Indiana Univ. Math. J. 22, 577-593 (1972). Errata: Indiana Univ. Math. J. 25, 301 (1976).

[18] J. W. Evans, Nerve axon equations. IV. The stable and unstable impulse, Indiana Univ. Math. J. 24, 1169-1190 (1975).

[19] H. Freistühler and P. Szmolyan, Spectral stability of small shock waves, Arch. Ration. Mech. Anal. 164, 287-309 (2002).

[20] R. A. Gardner, On the structure of the spectra of periodic travelling waves, J. Math. Pures Appl. 72, 415-439 (1993).

[21] R. Gardner, Instability of oscillatory shock profile solutions of the generalized BurgersKdV equation, Physica D 90, 366-386 (1996).

[22] R. A. Gardner, Spectral analysis of long wavelength periodic waves and applications, J. reine angew. Math. 491, 149-181 (1997).

[23] R. A. Gardner and C. K. R. T. Jones, Travelling waves of a perturbed diffusion equation arising in a phase field model, Indiana Univ. Math. J. 39, 1197-1222 (1989).

[24] R. A. Gardner and K. Zumbrun, The gap lemma and geometric criteria for instability of viscous shock profiles, Commun. Pure Appl. Math. 51, 797-855 (1998).

[25] F. Gesztesy and H. Holden, A unified approach to eigenvalues and resonances of Schrödinger operators using Fredholm determinants, J. Math. Anal. Appl. 123, 181198 (1987).

[26] F. Gesztesy, Y. Latushkin, and K. A. Makarov Evans functions and modified Fredholm determinants, Report No. 36/2004, Oberwolfach Reports, 1, 1946-1948 (2004).

[27] F. Gesztesy, Y. Latushkin, M. Mitrea, and M. Zinchenko, Non-self-adjoint operators, infinite determinants, and some applications, preprint, 2005.

[28] F. Gesztesy and K. A. Makarov, (Modified) Fredholm Determinants for Operators with Matrix-Valued Semi-Separable Integral Kernels Revisited, Integral Eq. Operator Theory 47, 457-497 (2003). (See also Erratum 48, 425-426 (2004) and the corrected electronic only version in $\mathbf{4 8}, 561-602(2004)$.)

[29] I. Gohberg, S. Goldberg, and N. Krupnik, Hilbert-Carleman and regularized determinants for linear operators, Integr. Eq. Operator Theory 27, 10-47 (1997).

[30] I. Gohberg, S. Goldberg, and N. Krupnik, Traces and Determinants of Linear Operators, Operator Theory: Adv. Appl., Vol. 116, Birkhäuser, Basel, 2000

[31] I. Gohberg and M. G. Krein, Introduction to the Theory of Linear Nonselfadjoint Operators, Translations of Mathematical Monographs, Vol. 18, Amer. Math. Soc., Providence, RI, 1969

[32] O. Guès, G. Métivier, M. Williams, and K. Zumbrun, Existence and stability of multidimensional shock fronts in the vanishing viscosity limit, Arch. Ration. Mech. Anal. 175, 151-244 (2005). 
[33] P. Hartman, Ordinary Differential Equations, corr. reprint of the 2nd ed., SIAM, Philadelphia, PA, 2002.

[34] P. Howard and K. Zumbrun, The Evans function and stability criteria for degenerate viscous shock waves, Discrete Contin. Dyn. Syst. 10, 837-855 (2004).

[35] C. K. R. T. Jones and T. Kapitula, Stability of fronts, pulses, and wave-trains. Preprint, 2003.

[36] R. Jost and A. Pais, On the scattering of a particle by a static potential, Phys. Rev. 82, 840-851 (1951).

[37] T. Kapitula, The Evans function and generalized Melnikov integrals, SIAM J. Math. Anal. 30, 273-297 (1998).

[38] T. Kapitula and B. Sandstede, Stability of bright solitary-wave solutions to perturbed nonlinear Schrödinger equations, Physica D 124, 58-103 (1998).

[39] T. Kapitula and B. Sandstede, Edge bifurcations for near integrable systems via Evans function techniques, SIAM J. Math. Anal. 33, 1117-1143 (2002).

[40] T. Kapitula and B. Sandstede, Eigenvalues and resonances using the Evans function, Discrete Contin. Dyn. Syst. 10, 857-869 (2004).

[41] Y. Latushkin and Y. Tomilov, Fredholm differential operators with unbounded coefficients, J. Diff. Eq. 208, 388-429 (2005).

[42] G. Lyng and K. Zumbrun, One-dimensional stability of viscous strong detonation waves, Arch. Ration. Mech. Anal. 173, 213-277 (2004).

[43] J. R. Miller and M. I. Weinstein, Asymptotic stability of solitary waves for the regularized long-wave equation, Commun. Pure Appl. Math. 49, 399-441 (1996).

[44] R. G. Newton, Relation between the three-dimensional Fredholm determinant and the Jost function, J. Math. Phys. 13, 880-883 (1972).

[45] R. G. Newton, Inverse scattering. I. One dimension, J. Math. Phys. 21, 493-505 (1980).

[46] R. G. Newton, Scattering Theory of Waves and Particles, 2nd ed., Dover, New York, 2002.

[47] M. Oh and K. Zumbrun, Stability of periodic solutions of conservation laws with viscosity: Analysis of the Evans function, Arch. Ration. Mech. Anal. 166, 99-166 (2003).

[48] K. Palmer, Exponential dichotomies and transversal homoclinic points, J. Diff. Eq. 55, 225-256 (1984).

[49] K. Palmer, Exponential dichotomy and Fredholm operators, Proc. Amer. Math. Soc. 104, 149-156 (1988).

[50] R. L. Pego and M. I. Weinstein, Eigenvalues, and instabilities of solitary waves, Philos. Trans. Roy. Soc. London Ser. A 340, 47-94 (1992).

[51] R. L. Pego and M. I. Weinstein, Evans' function, Melnikov's integral, and solitary wave instabilities, in Differential Equations with Applications to Mathematical Physics, W. F. Ames, E. M. Harrell II, J. V. Herod (eds.), Academic Press, Boston, 1993, pp. 273-286.

[52] R. L. Pego and M. I. Weinstein, Asymptotic stability of solitary waves, Commun. Math. Phys. 164, 305-349 (1994).

[53] D. Peterhof, B. Sandstede, and A. Scheel, Exponential dichotomies for solitary-wave solutions of semilinear elliptic equations on infinite cylinders, J. Diff. Eq. 140, 266-308 (1997).

[54] R. Plaza and K. Zumbrun, An Evans function approach to spectral stability of smallamplitude shock profiles, Discrete Contin. Dyn. Syst. 10, 885-924 (2004).

[55] M. Reed and B. Simon, Methods of Modern Mathematical Physics. IV: Analysis of Operators, Academic Press, New York, 1978.

[56] R. Sacker, The splitting index for linear differential systems, J. Diff. Eq. 33, 368-405 (1979).

[57] B. Sandstede, Stability of travelling waves, in Handbook of dynamical systems, Vol. 2, B. Hasselblatt and A. Katok (eds.), North-Holland, Elsevier, Amsterdam, 2002, pp. 983-1055.

[58] B. Sandstede and A. Scheel, Evans function and blow-up methods in critical eigenvalue problems, Discrete Contin. Dyn. Syst. 10, 941-964 (2004).

[59] B. Sandstede and A. Scheel, Absolute instabilities of standing pulses, Nonlinearity 18, 331-378 (2005)

[60] D. H. Sattinger, On the stability of waves of nonlinear parabolic systems, Adv. Math. 22, 312-355 (1976). 
[61] D. H. Sattinger, Weighted norms for the stability of traveling waves, J. Diff. Eq. 25, 130-144 (1977).

[62] D. Serre and K. Zumbrun, Boundary layer stability in real vanishing viscosity limit, Commun. Math. Phys. 221, 267-292 (2001).

[63] B. Simon, Quantum Mechanics for Hamiltonians Defined as Quadratic Forms, Princeton University Press, Princeton, NJ, 1971.

[64] B. Simon, On the number of bound states of two body Schrödinger operators - a review, in Studies in Mathematical Physics,, E. H. Lieb, B. Simon, A. S. Wightman (eds.), Princeton Univ. Press, Princeton, 1976, pp. 305-326.

[65] B. Simon, Notes on infinite determinants of Hilbert space operators, Adv. Math. 24, 244-273 (1977).

[66] B. Simon, Trace Ideals and their Applications, London Mathematical Society Lecture Notes Series 35, Cambridge University Press, Cambridge, 1979.

[67] K. Zumbrun, Multidimensional stability of planar viscous shock waves, in Advances in the Theory of Shock Waves, T.-P. Liu, H. Freistühler, and A. Szepessy (eds.), Progress Nonlin. Diff. Eqs. Appls., 47, Birkhäuser, Boston, 2001, pp. 307-516.

[68] K. Zumbrun, Planar stability criteria for viscous shock waves of systems with real viscosity, preprint, 2004, to appear in the CIME lecture notes series.

[69] K. Zumbrun and P. Howard, Pointwise semigroup methods and stability of viscous shock waves, Indiana Univ. Math. J. 47, 937-992 (1998). Errata: Indiana Univ. Math. J. 51, 1017-1021 (2002).

[70] K. Zumbrun and D. Serre, Viscous and inviscid stability of multidimensional planar shock fronts, Indiana Univ. Math. J. 48, 741-871 (1999).

Department of Mathematics, University of Missouri, Columbia, MO 65211, USA

E-mail address: fritz@math.missouri.edu

URL: http://www.math.missouri.edu/personnel/faculty/gesztesyf.html

Department of Mathematics, University of Missouri, Columbia, MO 65211, UsA

E-mail address: yuri@math.missouri.edu

$U R L:$ http://www.math.missouri.edu/personnel/faculty/latushkiny.html

Department of Mathematics, University of Missouri, Columbia, MO 65211, UsA

E-mail address: makarov@math.missouri.edu

$U R L:$ http://www.math.missouri.edu/personnel/faculty/makarovk.html 